\title{
Analysis and Interpretation of Complex Biological Signal Transduction Networks
}

Holly N. Currie

West Virginia University

Follow this and additional works at: https://researchrepository.wvu.edu/etd

\section{Recommended Citation}

Currie, Holly N., "Analysis and Interpretation of Complex Biological Signal Transduction Networks" (2014). Graduate Theses, Dissertations, and Problem Reports. 287.

https://researchrepository.wvu.edu/etd/287

This Dissertation is protected by copyright and/or related rights. It has been brought to you by the The Research Repository @ WVU with permission from the rights-holder(s). You are free to use this Dissertation in any way that is permitted by the copyright and related rights legislation that applies to your use. For other uses you must obtain permission from the rights-holder(s) directly, unless additional rights are indicated by a Creative Commons license in the record and/ or on the work itself. This Dissertation has been accepted for inclusion in WVU Graduate Theses, Dissertations, and Problem Reports collection by an authorized administrator of The Research Repository @ WVU.

For more information, please contact researchrepository@mail.wvu.edu. 


\title{
Analysis and Interpretation of Complex Biological Signal Transduction Networks
}

\author{
Holly N. Currie
}

\author{
Dissertation submitted \\ to the Eberly College of Arts and Sciences \\ at West Virginia University \\ in partial fulfillment of the requirements for the degree of \\ Doctor of Philosophy in \\ Chemistry
}

\author{
Submitted for Approval by \\ Jonathan W. Boyd, Ph.D., Chair \\ Lisa A. Holland, Ph.D. \\ Fred L. King, Ph.D. \\ Justin Legleiter, Ph.D. \\ J.P. O'Callaghan, Ph.D. \\ C. Eugene Bennett Department of Chemistry
}

\section{Morgantown, West Virginia \\ 2014}

Keywords: Graph Theory, Cell Signaling, Network, Phosphorylation, Toxicity, Stress Copyright 2014 Holly N. Currie 


\title{
ABSTRACT \\ Analysis and Interpretation of Complex Biological Signal Transduction Networks
}

\author{
Holly N. Currie
}

Cellular signaling is a complex system of biological communication that coordinates cellular activities via biochemical reactions. The passing of an extracellular signal across a cell membrane to intracellular signaling molecules is referred to as signal transduction. Signals transduced across a cell's membrane influence its function, and allow the cell to respond to its local environment. Within the cell, proteins are key responders to, and carriers of, the transduced signal. A class of proteins called kinases mediate most of the signal transduction in eukaryotic cells by catalyzing the phosphorylation of substrate proteins. The post-translational modification of proteins by phosphorylation regulates protein conformation, thereby influencing its function and many cellular processes. The multitude of interactions occurring between proteins within a cell form a complex signaling network that regulates and coordinates essentially all cellular activities, where reversible phosphorylation serves as a key means by which proteins can adjust the activity of other proteins. Understanding cellular signaling is a major challenge facing scientists today. The ability to decipher the complexity of cell signaling is necessary to thoroughly understand normal biological functions, the pathophysiology of diseases, and the effects of toxic exposures. A more intimate understanding of cellular signaling could facilitate the development of improved therapeutic strategies for many diseases and conditions. Advances in technology have facilitated the collection of large datasets describing cell signal transduction networks, but it is a challenge to integrate data describing many individual proteins into concise and meaningful biological knowledge. A complete understanding of cell signaling requires the ability to capture and integrate information pertaining to as much of the entire biological network as possible. This dissertation focuses on the development and application of an approach suitable for analyzing and interpreting the networked responses of cells and tissues to stress by monitoring the phosphorylation and upregulation of proteins. The approach utilized is grounded in the field of graph theory, and describes networked stress responses based solely on experimental condition-specific data. The approach's ability to describe the mode of action for an unknown toxic exposure in vitro is demonstrated. The approach is also utilized to depict low dose toxicant induced perturbations in the balance between mitogen activated protein kinase signaling pathways in vitro, providing an informative and sensitive means of assessing toxicological effects on biological systems. Finally, the analysis is used to investigate the complex networked response of muscle tissue to traumatic injury. 


\section{DEDICATION:}

This document is dedicated to my loving parents, Mark and Heidi Williams, for providing me with everything I have ever needed emotionally, physically, and spiritually.

\section{THANK YOU:}

To my husband, Stephen Currie - for your patience, ability to calm and comfort me, and for always believing in me.

To my sisters, Heather Lux and Kayla Williams - for your love and support.

To my grandmother Donna Cerullo - for your love and your enthusiasm for everything we grandchildren do. 


\section{ACKNOWLEDGEMENTS}

I would like to acknowledge my advisor, Dr. Jonathan Boyd, for his time, all the tough lessons, and for pushing me to become the person I am today.

I would like to acknowledge and thank my committee members, Dr. Jonathan W. Boyd, Dr. Lisa A. Holland, Dr. Fred L. King, Dr. Justin Legleiter, and Dr. James P. O'Callaghan for their time, mentorship, and support.

I would like to acknowledge Dr. Matthew S. Loos for the gift of his talents by performing the rat surgeries for this work.

I would like to thank Dr. Giovanni Scardoni for providing the modified CentiScaPe plug-in, without you this work would not be possible.

I would like to acknowledge my present lab mates, Julie A. Vrana and Alice A. Han for their input, companionship, willingness to grab lunch or coffee, and for providing comic relief when needed.

I would like to acknowledge my past lab mates, Stanley Strawbridge, Nicholas Rubenstein, Amber Cook, and Xueli Gao for their support. I am proud of the paths you have each chosen to pursue. 


\section{TABLE OF CONTENTS}

LIST OF FIGURES ---

LIST OF TABLES ---

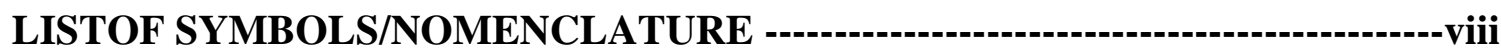

\section{Chapter 1}

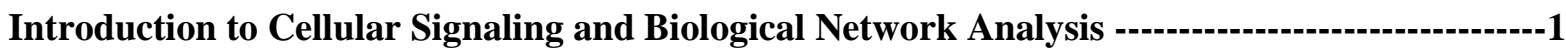

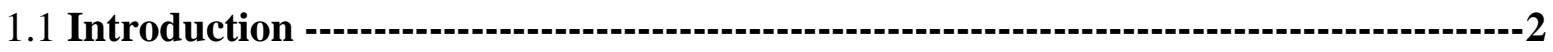

1.1.1. Cell Signaling ---_-

1.1.2. Kinases ---

1.1.3. Mitogen-activated Protein Kinases ---_-

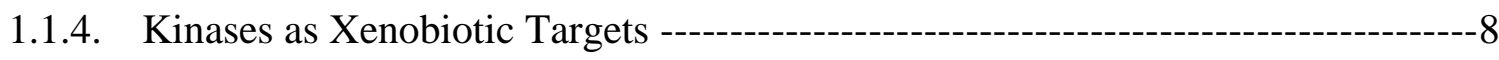

1.1.5. Monitoring Kinase Signaling ---_-

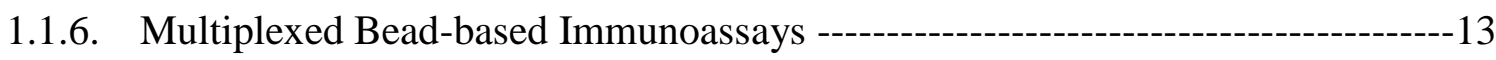

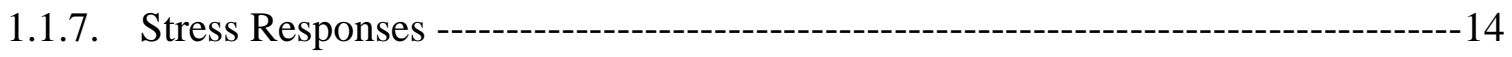

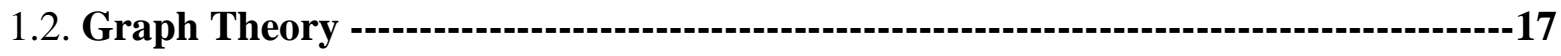

1.2.1. Graph Theory Basics ---_-

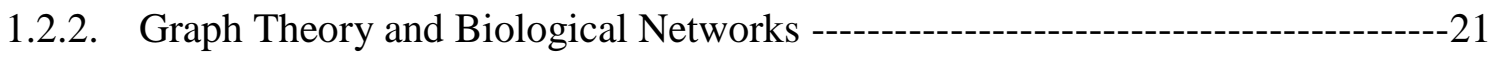

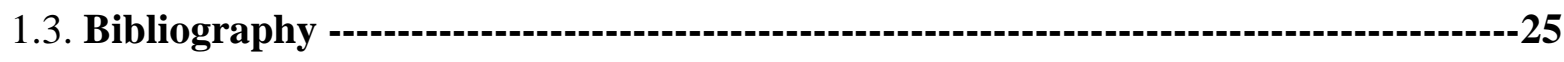

\section{Chapter 2}

Network Analysis Approach to Determine Xenobiotic Toxic Modes of Action --------------35

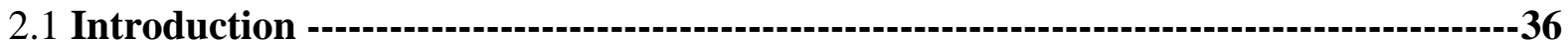

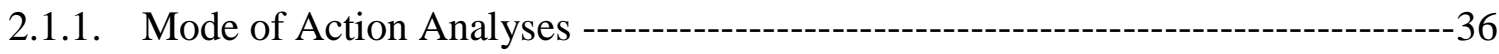

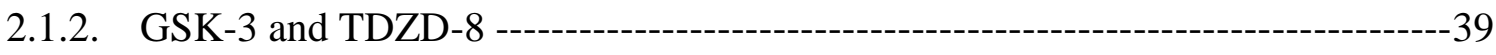

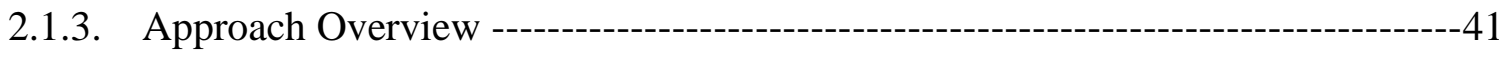


2.1.4. Radiality

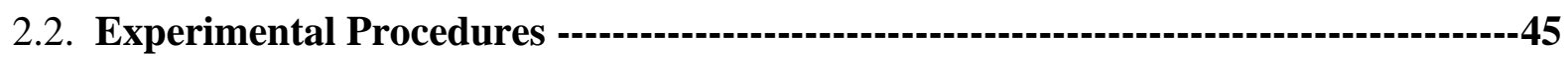

2.2.1. Materials --------------------------------------------------------------------------------------45

2.2.2. Cell Culture -------------------------------------------------------------------------------45

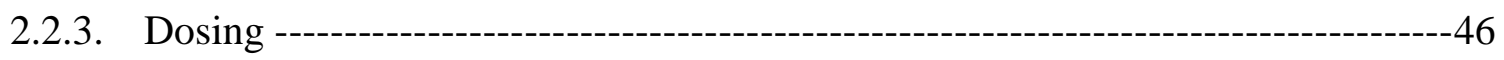

2.2.4. Multiplex Phosphoprotein Assay ---------------------------------------------------------46

2.2.5. Plasma Membrane Degradation Assay ------------------------------------------------47

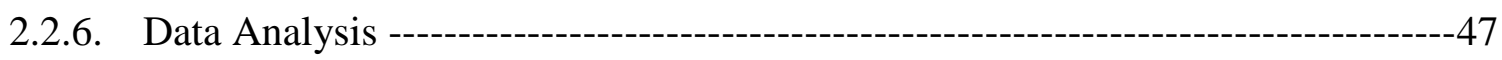

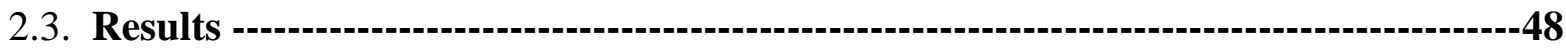

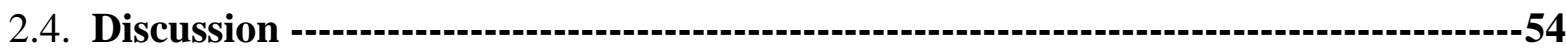

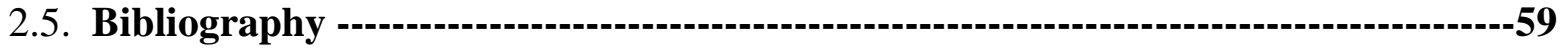

\section{Chapter 3}

Characterization of Network Perturbations in Response to Inhibitor Induced

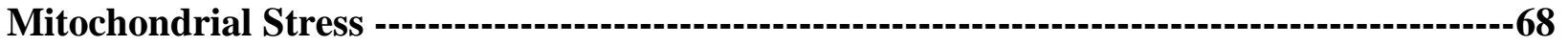

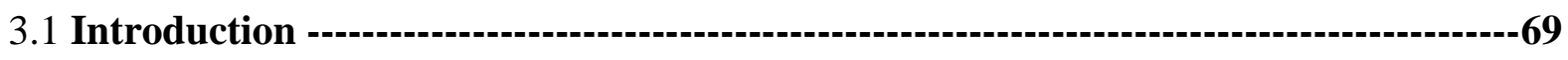

3.1.1. Mitogen Activated Protein Kinase Crosstalk, and Cell Death ----------------------69

3.1.2. Graph Theoretical Identification of Low-dose Network Perturbations ------------71

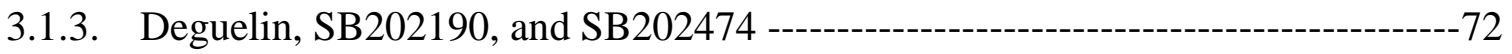

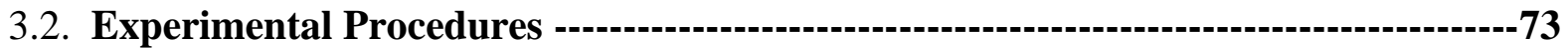

3.2.1. Materials ----------------------------------------------------------------------------------------'73

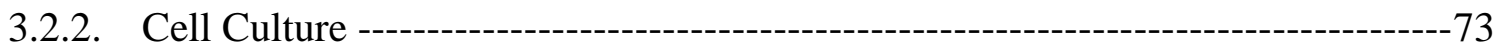

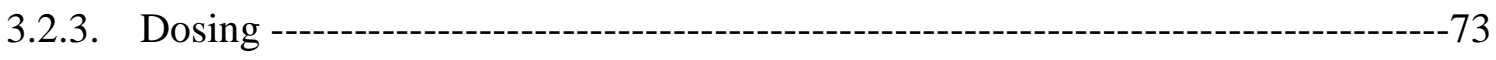

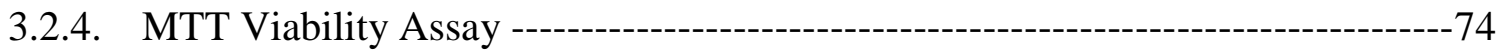

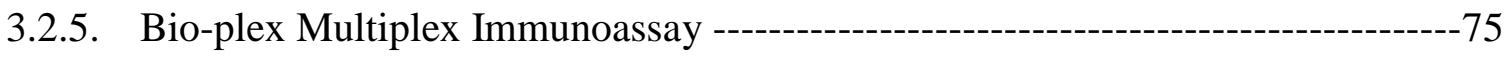


3.2.6. Data Analysis --------------------------------------------------------------------------------76

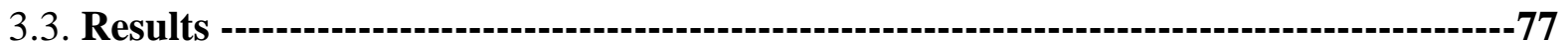

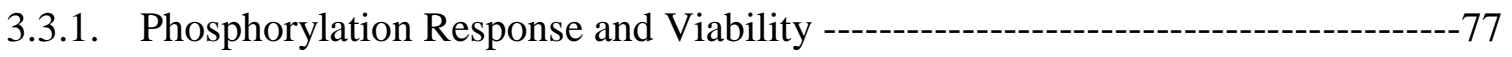

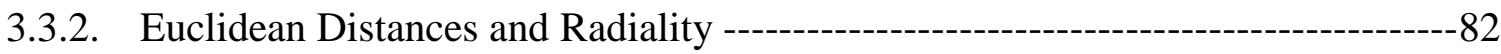

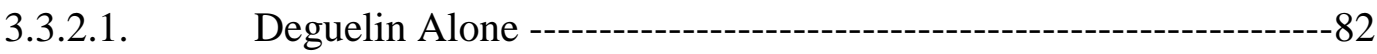

3.3.2.2. Deguelin in Combination with SB202190 -------------------------86

3.3.2.3. Deguelin in Combination with SB202474 ------------------------89

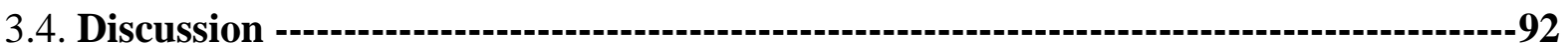

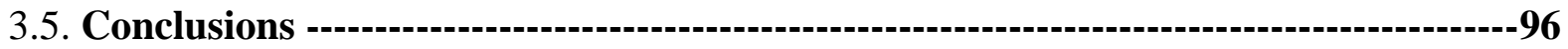

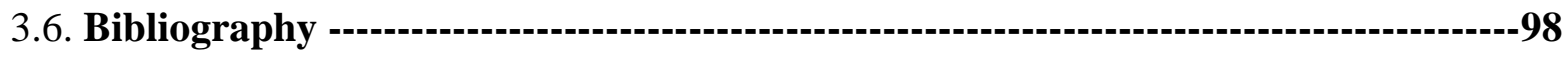

\section{Chapter 4}

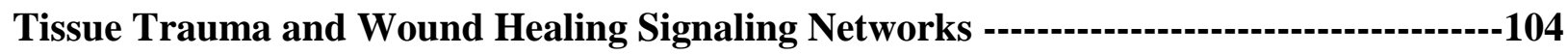

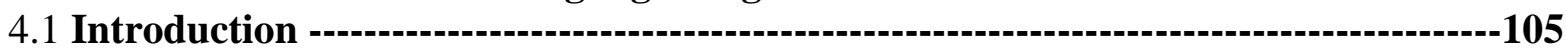

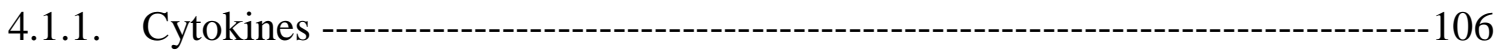

4.1.2. Tissue Trauma, Wound Healing, and Cytokines ---------------------------------107

4.1.3. Muscle Tissue Injury ---------------------------------------------------------------------108

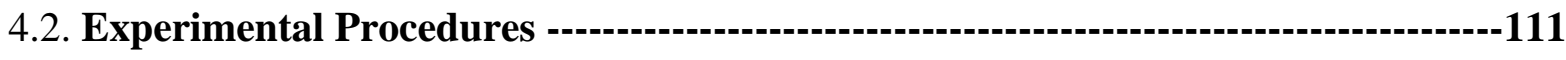

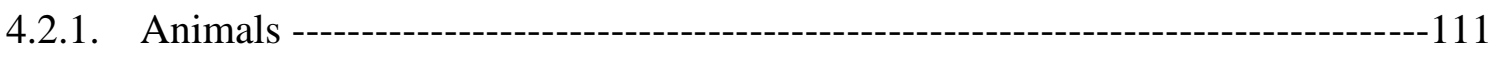

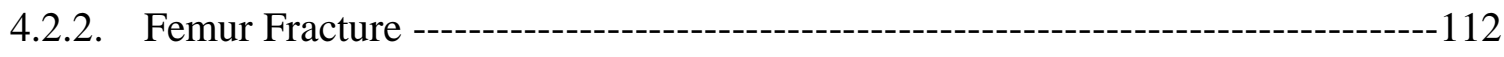

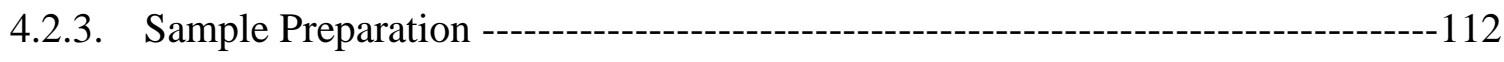

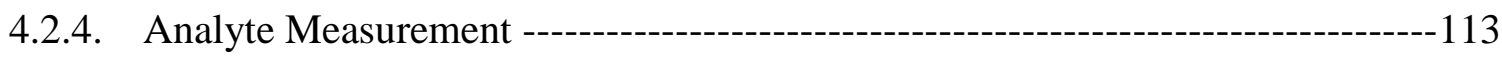

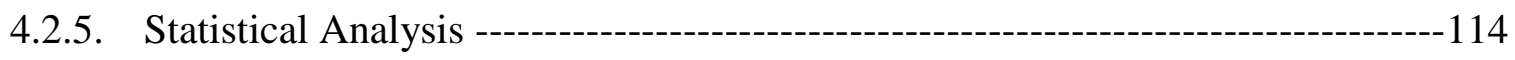

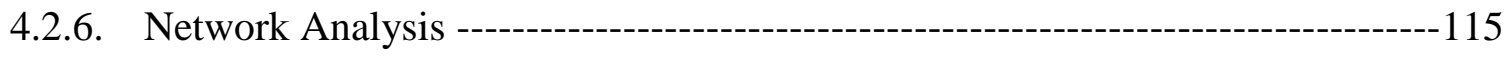

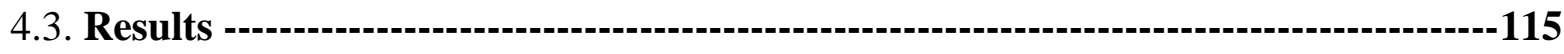




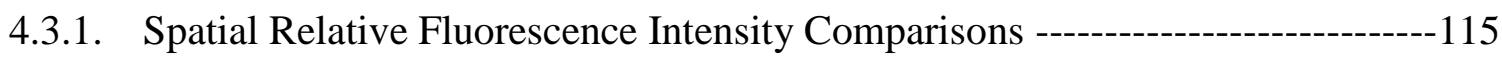

4.3.1.1. Cytokine Spatial Response Comparisons -----o----------------115

4.3.1.2. Phosphoprotein Spatial Response Comparisons ------------------118

4.3.1.3. Total Protein Spatial Response Comparisons -----------------121

4.3.2. Network Analysis ---

4.3.2.1. Radiality Comparisons at Fracture -------------------------------123

4.3.2.2. Radiality Comparisons $1 \mathrm{~cm}$ Away from Injury -------------125

4.3.2.3. Radiality Comparisons Uninjured Leg --- R

4.3.2.4. Across Locations Radiality Comparisons -----------------------128

4.3.2.5. Across Time Radiality Comparisons - $\quad$ -

4.4. Discussion ---131

4.4.1. Spatial Protein Responses to Injury --------------------------------------------131

4.4.2. Network Response to Tissue Injury ---------------------------------136

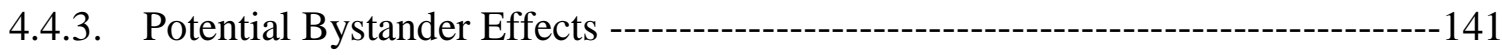

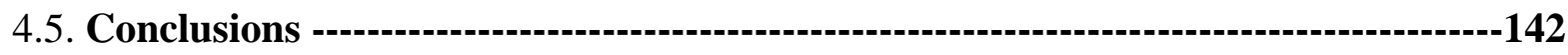

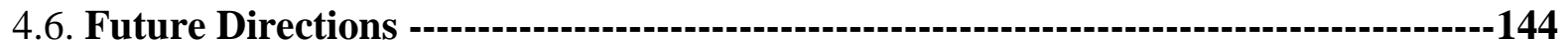

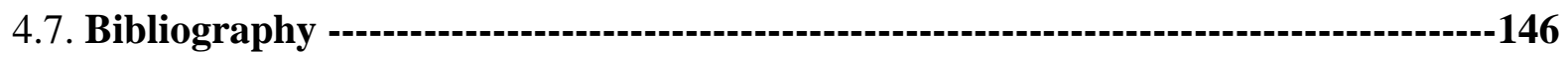




\section{LIST OF FIGURES}

\section{Chapter 1 Figures}

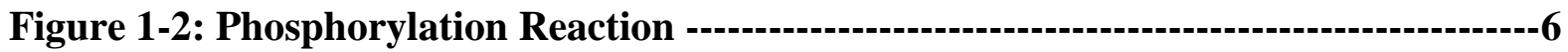

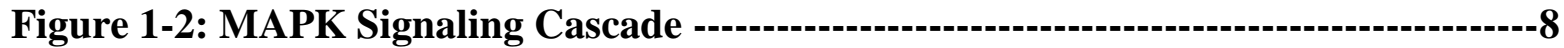

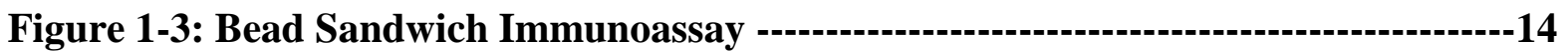

\section{Chapter 2 Figures}

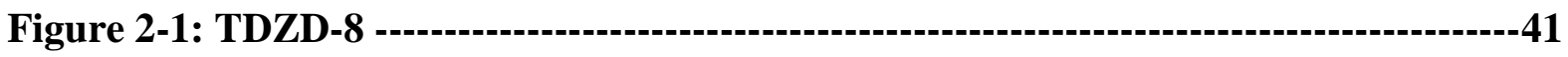

Figure 2-2: Relative Plasma Degradation in Response to TDZD-8 Exposure ------------49

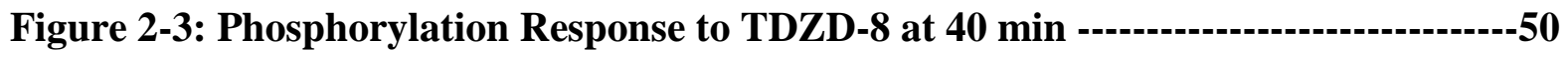

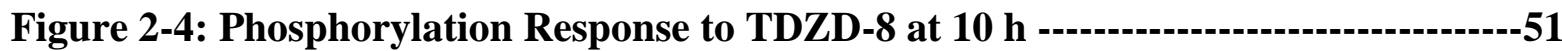

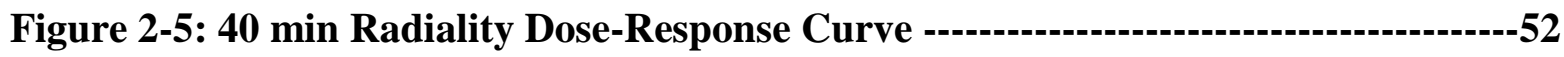

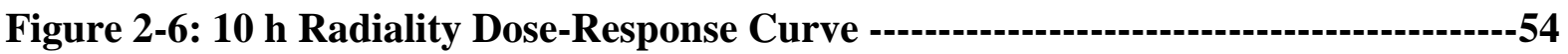

\section{Chapter 3 Figures}

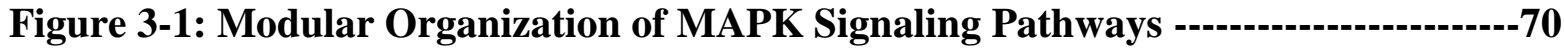

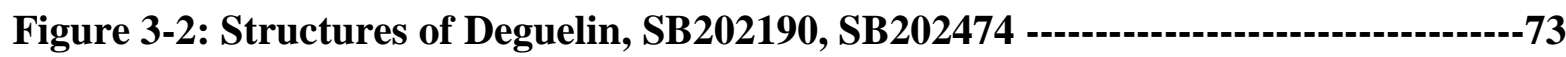

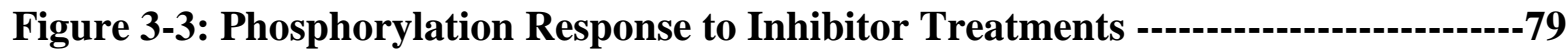

Figure 3-4: Relative Viability in Response to Inhibitor Treatments ----------------------81

Figure 3-5: Network Graphs for $10 \mu \mathrm{M}$ Deguelin Exposure ---------------------------83

Figure 3-6: Deguelin Alone Normalized Radiality Dose-response Curves for JNK,

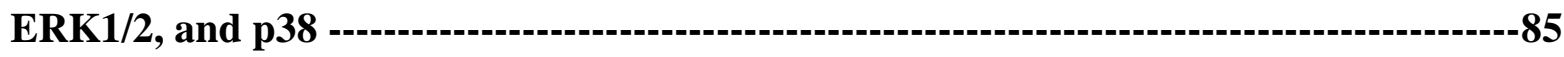


Figure 3-7: Deguelin Alone Normalized Radiality Dose-response Curves for Akt, HSP27, IkB $\alpha$, p53, and p90RSK 85

Figure 3-8: Deguelin in Combination with SB202190 Normalized Radiality Doseresponse Curves for JNK, ERK1/2, and p38 88

Figure 3-9: Deguelin in Combination with SB202190 Normalized Radiality Doseresponse Curves for Akt, HSP27, IkB $\alpha$, p53, and p90RSK 88

Figure 3-10: Deguelin in Combination with SB202474 Normalized Radiality Doseresponse Curves for JNK, ERK1/2, and p38 91

Figure 3-11: Deguelin in Combination with SB202474 Normalized Radiality Doseresponse Curves for Akt, HSP27, IkB $\alpha$, p53, and p90RSK $-92$

\section{Chapter 4 Figures}

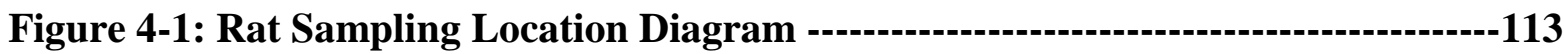

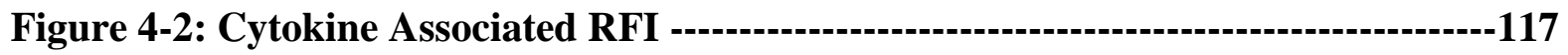

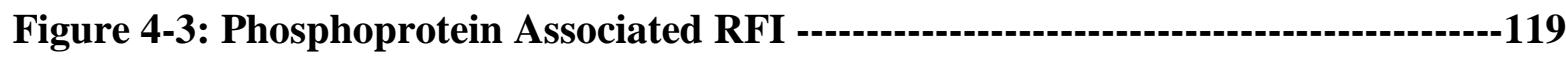

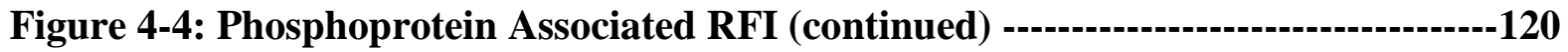

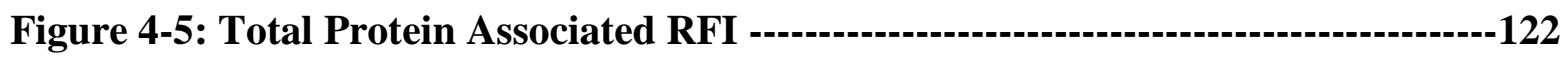

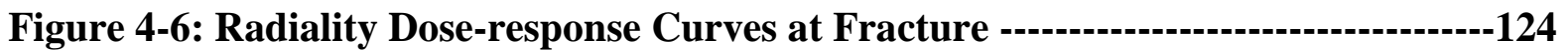

Figure 4-7: Radiality Dose-response Curves $1 \mathrm{~cm}$ Away from Fracture --------------126

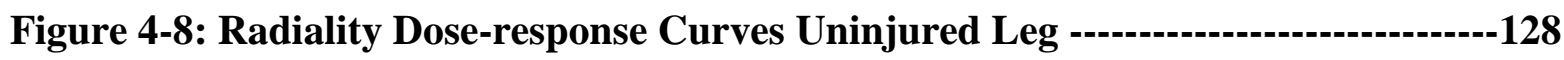

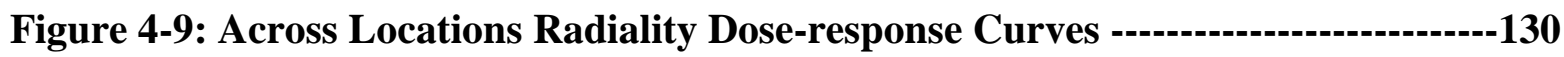

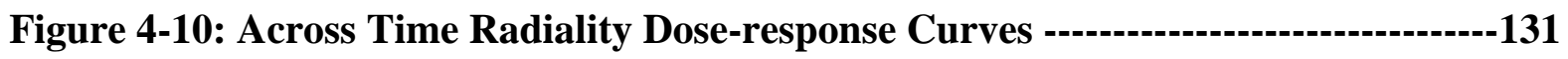

Figure 4-11: Schematic Depicting Network Analysis Results -------------------------137 


\section{LIST OF TABLES}

Chapter 2

Table 2-1: 40 min TDZD-8 Mean Radiality 52

Chapter 3

Table 3-1: Significant Differences in Phosphorylation Response -----------------------80

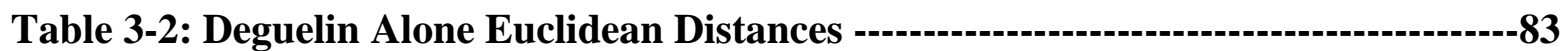

Table 3-3: Deguelin + 350 nM SB202190 Euclidean Distances ---------------------------87

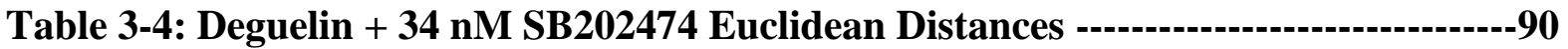




\section{LIST OF SYMBOLS/NOMENCLATURE}

1. ANOVA - analysis of variance

2. ARDS - acute respiratory distress syndrome

3. ATP - adenosine triphosphate

4. BAD - Bcl-2 associated death protein

5. BSA - Bovine Serum Albumin

6. CREB - cAMP response element-binding protein

7. DMEM - Dulbecco's Modified Eagle Medium

8. DMSO - dimethylsulfoxide

9. EGFR - epidermal growth factor receptor

10. ER - endoplasmic reticulum

11. ER Theory - Erdos Renyi Theory

12. ERK - extracellular signal regulated kinase

13. EthD-1 - ethidium homodimer

14. FBS - Fetal Bovine Serum

15. FRET - Förster Resonance Energy Transfer

16. GM-CSF - granulocyte-macrophage colony stimulating factor

17. GS-1 - glycogen synthase 1

18. GSK - glycogen synthase kianse

19. $\mathbf{h}$ - hour

20. HepG2 - human hepatocellular carcinoma cells

21. HSP27 - heat shock protein 27

22. $\mathbf{I C}_{\mathbf{5 0}}-50 \%$ inhibitory concentration

23. ICAM - intercellular adhesion molecule 1

24. IGFR - insulin-like growth factor 1 receptor

25. IL - interleukin

26. IRS1 - insulin receptor substrate 1

27. JNK - c-Jun NH(2)-terminal kinase

28. MAPK - mitogen activated protein kinase

29. min - minute

30. MIP-1 $\boldsymbol{\alpha}$ - macrophage inflammatory protein 1 alpha

31. MKK - mitogen activated protein kinase kinase

32. MKKK - mitogen activated protein kinase kinase kinase

33. MOA - mode of action

34. MOF - multiple organ failure

35. mRNA - messenger ribonucleic acid

36. MSK1 - mitogen and stress activated protein kinase 1

37. $\mathbf{m T O R}$ - mammalian target of rapamycin

38. MTT - 3-(4,5-dimethylthiazolyl-2)-2,5-diphenyltetrazolium bromide

39. NADH - Nicotinamide Adenine Dinucleotide

40. NADPH - Nicotinamide Adenine Dinucleotide Phosphate

41. NF-kB - nuclear factor kappa-light-chain-enhancer-of activated B cells

42. PARP - poly ADP ribose polymerase

43. PBS - phosphate buffered saline

44. PMSF - Phenylmethanesulfonylfluoride 
45. Pro - proline

46. QSAR - quantitative structure activity relationship

47. RFI - relative fluorescence intensity

48. ROS - reactive oxygen species

49. SD - standard deviation

50. SEM - standard error of the mean

51. Ser - serine

52. STAT - signal transducer and activator of transcription 2

53. TDZD-8 - 4-Benzyl-2-methyl-1,2,4-thiadiazolidine-3,5-dione

54. Thr - threonine

55. TNF- $\boldsymbol{\alpha}-$ tumor necrosis factor alpha

56. Tyr - tyrosine

57. VCAM - vascular adhesion molecule 


\section{Chapter 1}

Introduction to Cellular Signaling and Biological Network Analysis 


\subsection{Introduction}

An essential and central aspect of cellular life is that cells must constantly sense and respond to their local environment. An ultimate goal of experimental and theoretical biology is to be able to understand and predict how cells will respond to changes in their environment. Eukaryotic response to environmental factors is coordinated by intricate cellular signaling networks, which allow cells to accomplish basic cellular tasks such as growth, death, and differentiation. Understanding cellular signaling is a major challenge facing scientists today. The ability to decipher the complexity of cell signaling is necessary to thoroughly understand normal biological functions as well as the pathophysiology of diseases, and could facilitate the development of improved therapeutic strategies for many diseases and conditions.

Previous approaches used to study the biochemical signaling mechanisms responsible for the orchestration of biological processes have primarily focused on the isolated study of particular genes or proteins believed to be associated with distinct functions or conditions of interest. ${ }^{l}$ Recent technological advances and increased computational power have exposed the complex and dynamic networked nature of cellular signaling, making it evident that studying isolated portions of biological systems can lead to an under informed and even faulty understanding of the system. ${ }^{2,3}$ This is because all components of biological systems are connected as a network, making it possible for even distant components to have an effect on each other. Individual elements of biological systems do not function alone, and are not solely responsible for the control of a biological process or response. Biological functions are carried out by a coordinated effort between multitudes of network components. The architectures and regulatory mechanisms that govern network responses are extremely complex. Events or perturbations occurring at particular locations within these networks often have unpredictable 
effects at other localities within the network. The ability to forecast and understand networked biological responses to environmental stimuli is a challenging goal, important to the progression of many fields including molecular biology, pharmacology, and toxicology. ${ }^{4-6}$ There is a current need for network analysis approaches that are capable of describing the collective functioning of the expansive number of network components that comprise biological systems.

A complete understanding of cell signaling requires the ability to capture and integrate information pertaining to as much of the entire biological network as possible. This body of work focuses on the development and application of an approach suitable for analyzing and interpreting the networked responses of cells and tissues to stress. The approach utilized is grounded in the field of graph theory. Chapter 1 discusses the basics of cell signaling, the cellular stress response, techniques used to study cell signaling, and the utility of graph theory for the analysis of biological networks. Chapter 2 presents the application of the network analysis to determine xenobiotic toxic modes of action in HepG2 cells. Chapter 3 concerns the analysis and interpretation of the networked cellular response to mitochondrial stress. Chapter 4 covers the expansion the analysis to the more complex network response of tissue to traumatic injury stress. This work covers research that has been published in:

Currie, H.N., Vrana, J.A., Han, A.A., Scardoni, G., Boggs, N., Boyd, J.W. An Approach to Investigate Intracellular Protein Network Responses. Chemical Research in Toxicology. 2014 27(1), pp 17-26.

Currie, H.N., Loos, M.S., Vrana, J.A., Dragan, K., Boyd, J.W. Spatial Cytokine Distribution Following Traumatic Injury. Cytokine. 2014. 66(2), pp 112-118. 


\subsubsection{Cell Signaling}

Cell signaling is the complex system of communication and information flow present within living organisms that governs virtually all cellular activities including proliferation, differentiation, and cell death. ${ }^{7}$ Simply stated, cell signaling is the way in which cells perceive and respond to their environment. In cellular systems, extracellular signals (released from other cells or present in the organism's environment) in the form of hormones, steroids, xenobiotics, protein ligands (cytokines/growth factors), or mechanical forces are converted into intracellular signals at target cells through interactions with cell surface receptor proteins in a process referred to as signal transduction.

Transmembrane receptor proteins typically span the cell membrane having an extracellular and intracellular domain. ${ }^{8}$ The interaction between the ligand and receptor induces a conformational change in the receptor protein that leads to transmission of a signal across the plasma membrane via alteration of the activity of the intracellular portion of the receptor towards an intracellular signaling molecule. This triggers an intracellular signal transduction cascade where the activated intracellular signaling molecule goes on to interact with other molecules, typically signaling proteins, which further interact with other signaling molecules, and so on. Through this series of signaling interactions the cascade may eventually reach a protein, that once activated, will translocate from the cytoplasm to the nucleus where, through interactions with transcription factor proteins, the gene expression of the cell is altered. Signaling molecule interactions can lead to activation or deactivation of protein activities. ${ }^{9}$ Individual proteins can have multiple interaction sites that result in different protein functionalities. ${ }^{10,11}$ Cells constantly and simultaneously receive numerous signals which affect multiple intersecting and overlapping signal transduction pathways. These pathways form a complex network that allows cells to 
integrate multiple environmental signals, and respond with outputs specifically tailored to the environmental conditions.

\subsubsection{Kinases}

One of the largest, most prevalent, and well studied groups of signaling proteins are the protein kinases. $^{12}$ There are more than 518 known human protein kinases accounting for approximately $1.7 \%$ of the human genome. ${ }^{13,14}$ Protein kinases are intracellular enzymes that catalyze the transfer of the $\gamma$-phosphate group from abundant ATP molecules within the cell, to the hydroxyl groups on serine, threonine, or tyrosine residues of specific protein substrates (often

other kinases). ${ }^{15}$ This phosphorylation reaction is shown in Figure 1-1. The addition of a phosphate group covalently modifies the substrate protein, changing hydrophobic regions to hydrophilic, thereby altering the protein's conformation. ${ }^{16}$ The conformational change can alter the substrate protein's function in several ways, including activation or deactivation of its enzymatic activity, blocking binding sites for other proteins, or affecting its stability. ${ }^{12}$ Phosphorylation is a highly effective, reversible way of regulating the protein activities, that mediates the majority of signal transduction in eukaryotic cells, and controls almost all cellular processes including metabolism, transcription, cell cycle progression, cytoskeletal rearrangement, cell movement, apoptosis, and differentiation. ${ }^{13}$ 


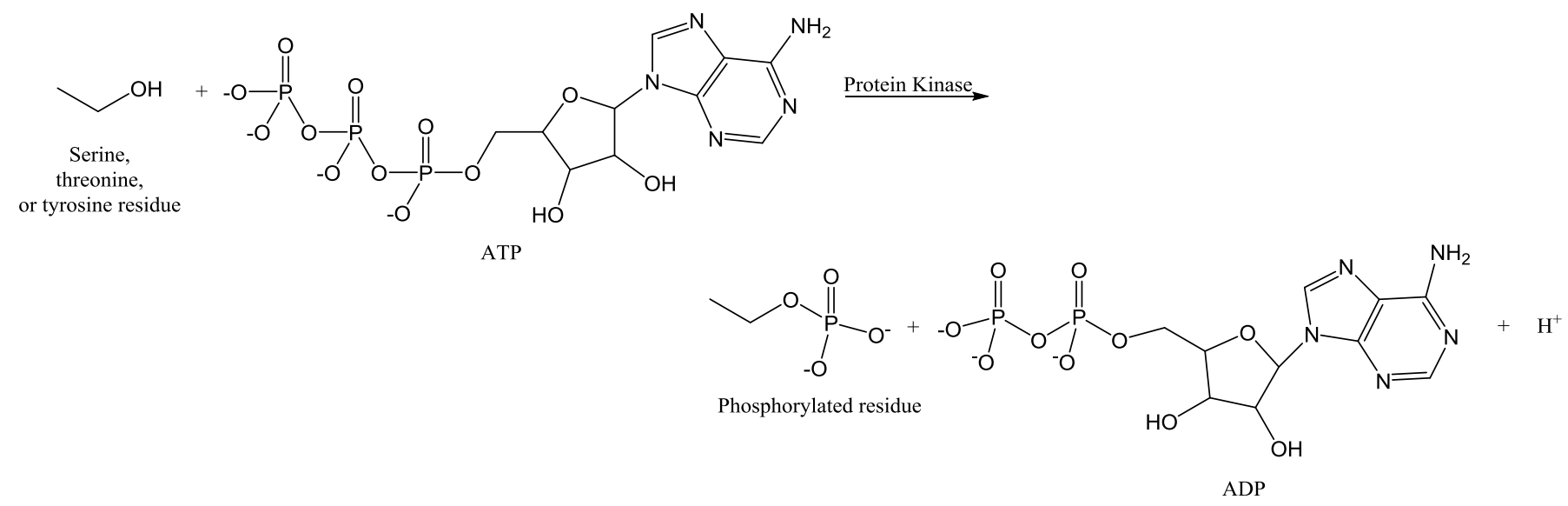

Figure 1-1. Phosphorylation reaction

Protein kinases are activated when induced conformational changes cause their catalytic domains to become accessible to both ATP and substrate proteins. The amino acid residues in the catalytic domain work to precisely position the ATP molecule so that the $\gamma$-phosphate group is aligned to facilitate its transfer to a hydroxyl group on the substrate protein. ${ }^{14}$ Activated kinases will only recognize and phosphorylate an amino acid if it is surrounded by a specific consensus amino acid sequence. ${ }^{14}$ The degree of specificity kinases have towards their substrates varies from kinase to kinase. ${ }^{17}$ Some kinases will only phosphorylate a single protein or a few closely related proteins, while other multifunctional kinases can target many different proteins. The specificity is primarily determined by the amino acid residue interaction between the catalytic domain of the acting kinase and the amino acid residues near the phosphorylation site of the substrate protein. However, based upon quaternary structure, distant residues can sometimes also contribute to the specificity. ${ }^{17}$

Protein phosphatases can regulate kinase activities by catalyzing the hydrolytic removal of phosphate groups from proteins. Protein phosphorylation and dephosphorylation are essentially irreversible under physiological conditions, and take place at negligible rates in the absence of enzymes. ${ }^{18}$ Thus, protein phosphorylation and dephosphorylation typically only take 
place by way of the action of specific protein kinases (at the expense of ATP cleavage) or phosphatases, respectively. This cycle of phosphorylation and dephosphorylation forms a complicated and sophisticated signaling network of kinases and other effector molecules that is essential to the orchestration of many important biological processes. ${ }^{13}$

\subsubsection{Mitogen-activated Protein Kinases}

The mitogen-activated protein kinases (MAPKs) are a group of kinases that have been extensively studied due to their fundamental involvement in a wide range of cellular processes including cell growth, differentiation, death, survival, and the immune response. ${ }^{19}$ The deregulation of MAPK signaling is associated with many diseases such as cancer, inflammatory disease, and diabetes. ${ }^{20}$ MAPKs are conserved in eukaryotes, and are among the oldest signal transduction pathways essential to many physiological processes throughout evolution. ${ }^{21}$

MAPKs allow cells to respond to a variety of extracellular stimuli including growth factors, hormones, cytokines, neurotransmitters, and xenobiotics. They form signal transduction cascades that respond to receptor level stimulation, and transfer signals to targets in both the cytosol and nucleus. The binding of ligands to transmembrane receptors leads to the activation of small Ras/Rho family GTP proteins that activate the first kinase in the MAPK cascade referred to as a MAPK kinase kinase (MKKK). ${ }^{21,22}$ This MKKK phosphorylates and activates a MAPK kinase (MKK) on either Ser or Thr residues. ${ }^{21,22}$ The activated MKK then goes on to dually phosphorylate a MAPK on Thr and Tyr residues leading to its activation. ${ }^{21,22}$ Activated MAPKs bind to specific docking sites on their target proteins and phosphorylate them at Ser or Thr residues that are usually followed by a Pro residue. ${ }^{19}$ MAPK signaling modules can have interactions with many other regulatory proteins such as phosphatases and scaffold proteins. ${ }^{23}$ 
Scaffold proteins can act to co-localize two MAPKs to facilitate their interaction, and represent a mechanism for regulating MAPK signaling. Activated MAPKs can translocate into the nucleus where they can phosphorylate proteins involved in transcriptional regulation. ${ }^{19}$ A simple schematic of a MAPK signaling cascade can be seen in Figure 1-2.

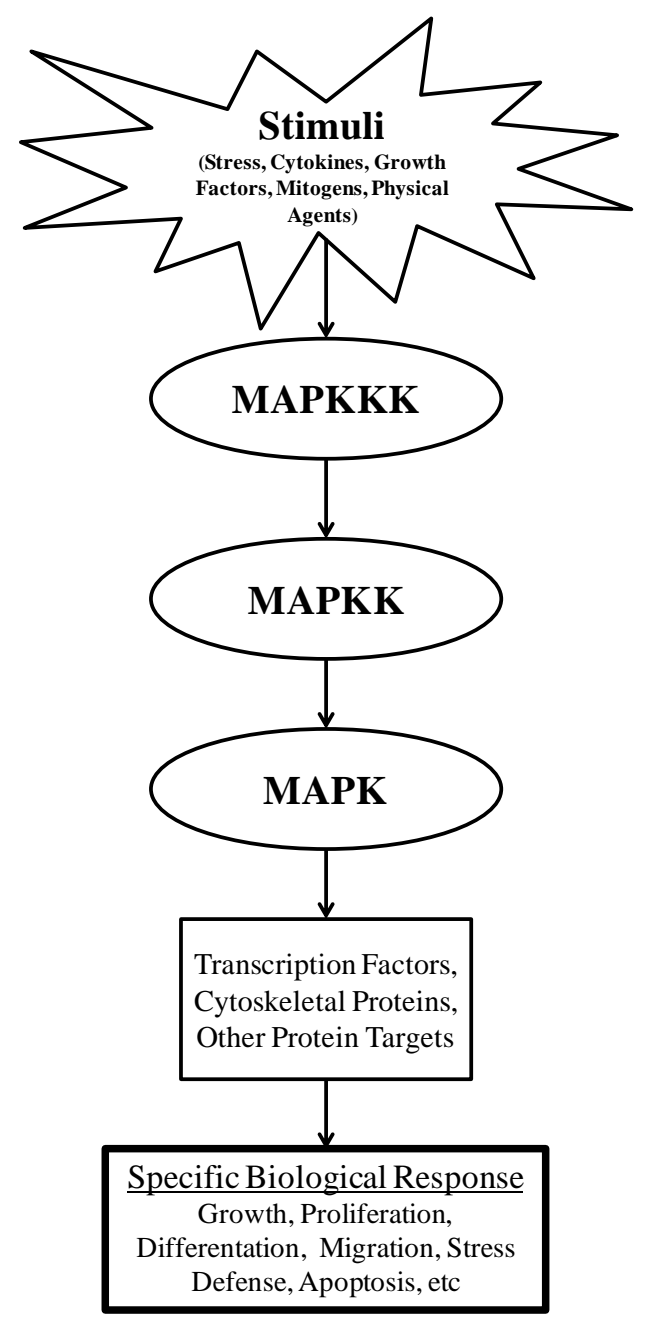

Figure 1-2. MAPK signaling cascade.

\subsubsection{Kinases as Xenobiotic Targets}

Alterations in normal kinase functioning and subsequent perturbation of protein kinase signaling has been related to pathological changes and diseased states such as cancer, diabetes, atherosclerosis, inflammatory diseases, Parkinson's disease, and Alzheimer's, making kinases 
popular therapeutic targets. ${ }^{8,24}$ Many drugs are designed to target specific kinases by exploiting their differences and binding to unique structural features. This binding interferes with the kinase's normal signaling activities, and offers a means of modulating the kinase's functioning. ATP competitive inhibitors are small molecules designed to competitively bind to and occupy the ATP-binding pocket within a kinase's catalytic domain, thereby preventing the kinase from phosphorylating other proteins. Substrate competitive inhibitors mimic substrate proteins, thereby preventing the kinase from phosphorylating its endogenous substrate. ${ }^{25}$ Other inhibitors interact with regulatory protein domains preventing the kinase from becoming active. In addition to their use as therapeutics, kinase inhibitors are also widely used as probes to study the physiological functions of proteins. A caveat underlying the use of kinase inhibitors as therapeutic and investigative tools is in the inhibitor's specificity. Compounds can inhibit a number of different targets making it difficult to be certain that the intended target is the only one being directly affected. ${ }^{25-27}$

Traditionally, kinases were described as members of distinct linear signaling pathways. This made therapeutic strategies involving the modulation of a single target appear viable. ${ }^{28}$ However, single target therapeutic strategies have extremely high failure rates, and many kinase targeted drugs induce undesirable side effects that are difficult to foresee. ${ }^{29}$ These difficulties are reflected in the 30-year decline in pharmaceutical development productivity in spite of increased spending by the pharmaceutical industry, and the multitude of improvements in 'omics' technologies that have provided expansive datasets describing cellular functioning. ${ }^{30}$ This trouble in developing successful monotherapies can be partially attributed to the fact that kinases function as large, complex, and dynamic networks, rather than distinct linear pathways. ${ }^{28}$ The dynamic nature of these networks makes it challenging to predict how they will respond to any 
perturbation. The high degree of interconnectedness between network components makes it likely that altering one specific protein will affect others. Actions directed at specific targets can radiate throughout the network, and manifest as undesired drug side effects. ${ }^{6}$

Cell signal transduction networks have evolved over billions of years to be able to withstand a variety of insults and disruptions. This entails backup mechanisms that can compensate for compromised network components. It is the high degree of connectivity in kinase networks that imparts tolerance and robustness towards deleterious events directed at single or a few node components in biological systems. ${ }^{31}$ If one avenue of communication is compromised, there are many other possible routes available for the transmission of important signals necessary for normal functioning and survival. This is especially true in the case of cancer cells, and explains why it can be difficult to treat cancer with single target regimens. The underlying architecture of signaling networks affects their function, and knowledge of this architecture is necessary to develop better treatment strategies. Indeed, recent years have seen a shift in drug discovery from targeted discovery to network pharmacology. ${ }^{6,27,32-35}$ It is now realized that therapeutic strategies are in need of a more intelligent design, where diseases are treated as rewired or unbalanced networks instead of single mutated genes or proteins. ${ }^{27,} 36$ Current therapeutic approaches could be enhanced by assessing diseases at the network level, and by evaluating the entire network response to therapeutic treatments. This requires the development of methods capable of characterizing and understanding the context specific, dynamic, and complex functioning of biological networks. 


\subsubsection{Monitoring Kinase Signaling}

Signaling can be studied at the level of the whole organism, at the level of individual cells, or at the level of isolated proteins or peptides. There is currently no approach or method that can capture the entire range of kinase interactions occurring in real time in a given biological system. There are many different approaches to studying kinase signaling, each with their own advantages and disadvantages. ${ }^{37-44}$ The simplest level seeks to identify possible substrates of a kinase. The identification of biologically relevant kinase substrates has historically been a slow and laborious process that often yields unreliable results, ${ }^{37}$ and still remains a difficult task. Possible kinase substrates are often determined by incubating the isolated kinase of interest with putative substrate proteins or peptides in the presence of ATP and $\mathrm{Mg}^{2+}$, and then detecting which proteins/peptides became phosphorylated. These low throughput approaches can lead to the identification of kinase interactions that may not actually occur, because the behavior of interacting proteins can be significantly different in an intact biological system in comparison to when isolated. ${ }^{38}$ Just because a kinase can phosphorylate a target does not mean that it will in a living system. The use of highly concentrated purified kinases in these assays can cause a kinase to phosphorylate a substrate that it would not normally phosphorylate in vivo ${ }^{38}$. Removing a kinase from its cellular environment leads to a loss of physiological regulatory mechanisms. For example, if a third protein is necessary for the interaction between a kinase and its substrate to take place, the isolated analysis will not identify the interaction. X-ray crystallography can be used to visualize kinases bound to their substrates, and has contributed significantly to the current knowledge of kinase interactions. ${ }^{39}$ However, this only provides a static picture, and does not effectively capture the dynamics of phosphorylation processes in real systems. 
Other approaches seek to directly detect interacting kinases in living cells. Techniques used in these approaches include yeast-two-hybrid assays and Förster Resonance Energy Transfer (FRET). ${ }^{40,41}$ These approaches involve altering cells by genetically fusing proteins with large adducts which can lead to alterations in the studied protein's normal activity, and may not accurately reflect its activity. ${ }^{41,42}$ These techniques are also limited to studying a few kinases at a time making it difficult to capture network dynamics. Additionally, protein-protein interactions occurring during phosphorylation are considered transient with interacting proteins quickly dissociating following the phosphorylation event, making the event difficult to capture. ${ }^{12}$

Other less direct approaches primarily rely on determining the amount of each protein that is phosphorylated following varying degrees of stimulation or perturbation. This is commonly in the form of growth factors, kinase inhibitors, or genetic silencing/deletions. ${ }^{41-43}$ Kinase relationships are inferred based upon changes in the amount of each phosphorylated protein in response to the stimulation or perturbation. These approaches can monitor many more proteins than the more direct approaches, but cannot definitively identify which proteins are interacting. This is the type of approach utilized in this body of work.

Detecting phosphorylated proteins is also technically challenging due to the dynamic and reversible nature of phosphorylation, and the fact that the ratio of phosphorylated to unphosphorylated proteins in living systems is relatively low. ${ }^{16,43}$ The identification of the amount of phosphorylated protein does not reflect the connections between kinases or the role that they play in the signaling network. Elucidating the structure and function of kinase signaling networks is an especially challenging task. New technologies have facilitated the collection of large phosphoproteomic datasets revealing intricacies of cell signaling that were previously unknown, ${ }^{43}$ but this wealth of information presents issues in interpreting the data into 
meaningful and relevant knowledge. There is a current need for approaches that are capable of integrating this data to describe the networked response of kinases to stimulation or stress, and this body of work presents an approach used to concisely describe networked cell signaling responses based on condition-specific phosphorylation data.

\subsubsection{Multi-plexed Bead-based Immunoassays}

In the work presented here, a multiplexed bead-based immunoassay suspension array platform was used for targeted phosphoprotein analysis. This platform is attractive due to its ability to perform multiplexed analyses including as many as 100 protein targets, in $12.5 \mu 1$ of sample, in as little as $3 \mathrm{hrs,} \mathrm{and} \mathrm{with} \mathrm{a} \mathrm{relatively} \mathrm{simple} \mathrm{work} \mathrm{flow.}{ }^{44}$ The platform utilizes polystyrene microbeads that are uniquely coded with varying concentration ratios of two internal fluorescent dyes. ${ }^{45-47}$ Phospho-specific antibodies are immobilized on the surface of microbeads that have dye ratios designated to code for the particular target for which the antibody recognizes. Microbeads with different antibody coatings (and dye codes) are incubated with the sample in a single reaction well to achieve multi-analyte analysis. Following incubation, biotinylated secondary antibodies are added to the reaction well. Unbound secondary antibodies are washed away, and the microbeads are incubated with the fluorescent reporter, streptavidinphycoerythrin conjugate. Analysis is performed using a flow cytometer with lasers to identify the coded beads and to also detect the fluorescent phycoerythrin reporter. Figure 1-3 shows a representation of an analyte captured by the bead sandwich immunoassay. 


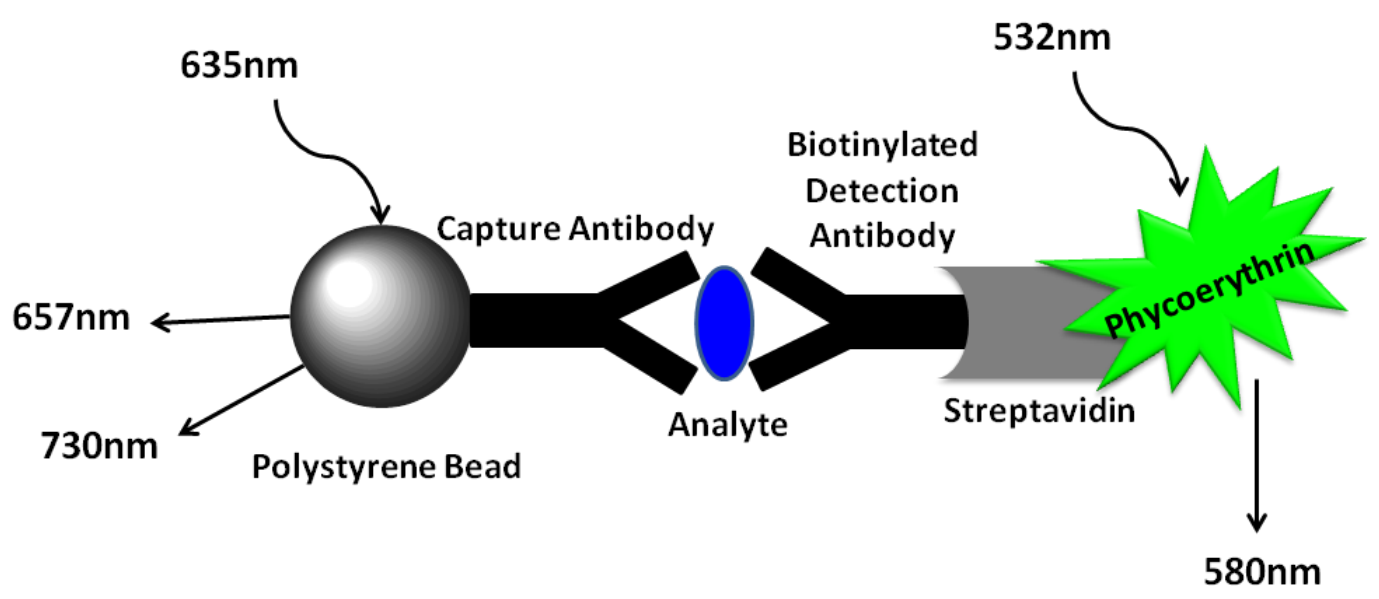

Figure 1-2. Bead sandwich immunoassay

The microbead platform was selected for the analyses performed in this body of work due to its fast, cost effective, simple work flow, availability of standardized protocols, and multiplexing capabilities necessary for analyzing network responses. This technology has been extensively optimized and validated and has a broad dynamic range. ${ }^{48}$ The suspension array platform was also chosen because cytokines were also targets of interest to this work. Cytokine concentrations can span a wide concentration range in biological samples, ${ }^{48,49}$ exceeding the dynamic range achievable by many instruments used in proteomic studies. Antibody based approaches including the microbead platform utilized here have suitable dynamic ranges for cytokine analysis. ${ }^{49}$ In the work presented here, the microbead multiplexed platform was used to identify changes in both total protein levels and phosphorylated protein levels in cells and tissues subjected to stress.

\subsubsection{Stress Responses}

Cells constantly sense their environments, which allows them to detect any type of stress and quickly adapt to survive. ${ }^{50-53}$ When whole organisms, organ systems, and tissues are subjected to environmental stressors, their responses are a direct result of the coordinated 
responses of individual cells mediated by signal transduction networks, and thus, cell signal transduction cascades are fundamental to stress responses. As a necessity for survival, all cells have developed defense mechanisms capable of responding to a wide variety of cellular threats. These mechanisms can be quite sophisticated and are referred to as cellular stress responses. Cellular stress responses have extraordinary physiological and pathological significance. A cell's fate after experiencing stress is dependent upon the type of cell, the proteome expressed at the time the stress is experienced, the nature of the stress, and the stress's magnitude and duration..$^{52,54}$

Eukaryotic cells have evolved to contain signal transduction networks capable of producing dynamic and coordinated responses that can alter virtually any cellular process to adapt to a perceived stress. ${ }^{50,52}$ Immediate responses are carried out by post-translational modifications such as phosphorylation, while long term adaptations are in the form of alterations to gene expression. ${ }^{50,52}$ Cellular stress responses frequently involve common evolutionarily conserved core stress response proteins, but can also utilize diverse molecular mechanisms. ${ }^{50,52,}$ 55, 56 Stress responses involving evolutionarily conserved stress proteins, such as heat shock proteins, are typically induced by deformation or damage to macromolecules, and aim to sense and mitigate protein damage, DNA damage, or altered cellular redox states. ${ }^{53,55,57}$ Many of these types of stress responses have been well characterized, ${ }^{54}$ and are sometimes rather simple in nature. For example heat shock or unfolded protein stress responses can be as simple as increasing the expression of chaperone proteins that assist damaged proteins in reestablishing their proper conformations.

Cells use multiple signaling pathways to respond to environmental changes and stresses, including survival signaling and destructive death signaling pathways. Survival signaling aims 
to push cells towards recovery while death signaling aims to remove cells that have been damaged beyond the point of recovery. Whether or not a cell will survive under conditions of stress is dependent on the network balance between survival promoting signaling and death promoting signaling, between which complicated interplay exists. ${ }^{58}$ The molecular mechanisms that control the balance between survival and death signaling and determine whether or not cells will survive under periods of stress are not fully elucidated. MAPK signaling is essential to the coordination of cellular responses to a wide variety of stresses including chemical exposure stress and physical stress. ${ }^{50,51,55-57,59-66}$ MAPK stress signaling is susceptible to regulatory input at all levels of their cascades via multiple mechanisms, including modification of MAPK subcellular localization, stimulus intensity, scaffold protein interactions, phosphatase activity, positive and negative feedback loops, and cascade crosstalk. ${ }^{56}$ Cell survival/death decisions are heavily influenced by MAPK signaling, and understanding this influence can provide valuable insight into the effects of stresses. ${ }^{57,67-69}$ However, the complex networked relationships between MAPKs, as well as the variety of ways MAPK signaling can be regulated makes it particularly difficult to decipher how MAPK signaling coordinates responses tailored to each of the many stresses a cell experiences.

The ability to predict how cells will respond to any type of stress is extremely desirable to many disciplines. Many aspects of the cellular stress response remain poorly understood, especially with regard to post-translationally regulated responses in human cells. ${ }^{52,53}$ This can be attributed to the current lack in understanding of the integrated functioning of the numerous signaling proteins present in human cells, as well as the variability that exists amongst different cell types. ${ }^{53}$ The numerous and diverse proteins that coordinate cellular responses, together comprise a complex cellular network through which information flows. A comprehensive 
understanding of how the flow of information through cellular networks coordinates their functioning and changes in order to sustain life remains elusive. The complexity of cellular signal transduction networks necessitates a global network analysis integrating comprehensive datasets to fully capture and understand how cells respond to stress. ${ }^{52,70}$ An understanding of cellular responses to stress is of profound importance when assessing toxicological risks and understanding disease pathophysiology.

The true complexity of cell signal transduction networks is now becoming apparent. It has become evident that more creative alternative network comprehensive approaches are needed to better investigate complex cell signaling. Networks are universal and important focal points of many disciplines. The field of mathematics referred to as graph theory has found widespread applicability in the study of complex networks across many disciplines. In the work presented here, graph theory was utilized to analyze and interpret signal transduction network responses to stress.

\subsection{Graph Theory}

Networks pervade all avenues of life from social circles, the internet, power grids, and food webs to the citation networks of scientists. ${ }^{71}$ Networks are inherently difficult to comprehend. A major challenge facing scientists today is how to accurately describe and understand how individual components of complex systems function together as a network. Analysis of the topology of complex networks can provide valuable insight into how complex networks operate. The topological organization of complex networks determines how information will flow throughout the system, and how the system functions. Networks are further complicated when the many connections between their elements are capable of changing, 
and the various connections have different strengths or weights. Due to their large size and complex interactions, describing the topological organization of many commonly encountered networks can be a lengthy and laborious task. ${ }^{72}$ Graph theory is a valuable mathematical modeling tool that can be used to characterize the global topological organization of networks.

Graph theory was initiated in 1736 by Leonhard Euler to solve the Seven Bridges of Königsberg problem. ${ }^{73}$ There were 7 bridges in the city of Königsberg that spanned various sections of the River Pregel. It was often questioned whether or not a path existed by which a person could cross each bridge only once and return home. Euler mathematically proved that it was in fact impossible to only cross each bridge once, and in doing so laid the foundation for graph theory. ${ }^{74}$ Since then, graph theory has been used to solve basic practical problems such as determining the maximal flow rate of water flowing from a source sink to a network of pipes, and more recently, to more involved and complex problems such as characterizing how group memberships (political, religious, or corporate board membership for example) within human networks affect the spread of attitudes and opinions. ${ }^{75}$

\subsubsection{Graph Theory Basics}

In graph theoretical analyses, a network of interacting elements is depicted as a graph consisting of nodes and edges. Nodes can represent any element contained within the network. Edges connect pairs of nodes, and represent the interaction or relationship (if one exists) between node pairs. Based on the connectivity of the graphs and any edge weightings, quantitative attributes that characterize the structure and behavior of networked systems can be calculated. ${ }^{75-}$ 79 Network edges can be directed or undirected. Directed edges depict relationships for which there is a known source node and target node. The Internet is an example of a network with 
directed edges. Networks in which the relationships between each node are mutual, such as human social networks, are undirected. Quantitative descriptors of connection strength or the amount of information flow between nodes can be utilized as edge weights. ${ }^{80}$ Quantitative parameters calculated using graph theory that concisely describe the topology of an entire network as a whole are referred to as network parameters. Other quantitative parameters, referred to as node centralities, describe the relevance of an individual node with regard to the rest of the network.

Pioneering work establishing the basic measures and techniques used in graph theory today was performed in the mid-twentieth-century by Erdos and Renyi. ${ }^{81}$ Erdos and Renyi found that probabilistic methods were quite useful in solving graph theory problems, and from their work, predicted that real complex networks could be modeled as random graphs. ${ }^{72,81}$ Random graphs are homogeneous graphs, where all nodes in the graph are wired together at random. This results in a binomial node degree distribution indicating that all nodes have approximately the same node degree (number of direct connections to other nodes). ${ }^{78}$ In the random graphs described by the Erdos Renyi (ER) theory, nodes with a relatively high connectivity or degree are practically absent. Random networks display small world characteristics, meaning that all nodes in a network can easily communicate with each other, either directly or through a relatively short path length or series of "go between" nodes. ${ }^{78}$ The small-world effect is the basis for a popular game called six degrees of Kevin Bacon in which players must connect Kevin Bacon to any given actor or actress through the shortest possible path of film collaborations. ${ }^{82,83}$ Small world characteristics allow for fast communication within networks. Following the development of ER theory, it was generally accepted that the majority of real complex networks 
encountered followed the principles of ER theory, but large datasets and the computational power needed to thoroughly test this theory were not available.

Recently, the availability of large datasets describing complex networks, the emergence of databases to upload and share this data, and increased computational power have allowed the random ER theory to be tested on networks spanning various disciplines. ${ }^{72}$ Two seminal papers were published that led to an explosive interest in the field of network science by changing the accepted views on real network structures. The first, published by Watts and Strogatz in 1998, proved that networks that are disordered, highly clustered, and non-random can have small world characteristics just as random graphs. Watts and Strogatz demonstrated that 3 real networks, the United States power grid, the collaboration graph of film actors, and the neural network of the worm, abided by the small world phenomenon. ${ }^{84}$ They suggested that most other real networks, including biological networks, likely display small world properties as well.

The second paper, published by Barabasi and Albert in 1999, demonstrated that networks across diverse fields of science share properties that do not fit the random ER graph model. ${ }^{72}$ They found that real networks display a high degree of self-organization, and that their node degree probability distributions decay as a power-law. Meaning that large networks organize into scale-free states, where a few nodes are likely to have a high connectivity or degree, while the majority of nodes in the network have a low connectivity. Barabasi and Albert demonstrated that the random graph models failed to represent real networks, because they did not account for growth and preferential attachment that occur in most real networks. Most real networks continuously grow by incorporating new nodes into their structures. When new nodes are added they preferentially attach to nodes in the network that already have a high degree of connectivity. Therefore, nodes with a high degree will continue to increase in connectivity while nodes with a 
low degree will remain low in connectivity. This realization, that many real networks display small world and scale-free properties, had considerable implications in the field of network science, because these types of network architectures could be used to explain why many real networks are functionally robust. ${ }^{75}$

\subsubsection{Graph Theory and Biological Networks}

Traditionally, biochemical interactions were thought of as individual linear pathways where enzymes acted upon substrates to form products. In the post-genomic era it became evident that many proteins interact with multiple other proteins in complex network structures rather than in a series of linear reactions, and are better modeled using graphs representing the networked nature of biochemical processes. ${ }^{85,86}$ In the past decade, many datasets describing molecular interactions (as well as maps of specific cellular networks) have been compiled, and graph theory has been applied to these datasets to characterize the properties of biological networks. $^{87-91}$

In the decade following the publication of the seminal papers by Watts and Barabasi, there was a flood of new research characterizing the topological structures of many real networks. ${ }^{92}$ This research has shown that many real networks, regardless of their function, have similar architectures. Biological networks are among these real networks found to have universal architectures. ${ }^{87}$ 92-95 Biological networks were shown to display small-world characteristics. This characteristic is important for efficient responses to network perturbations, and ensures that information can propagate throughout the network quickly even when some nodes have been compromised. ${ }^{94}$ The first demonstrated scale-free biological network was the worm neural network from the seminal paper published by Barabasi in $1999 .{ }^{72}$ In 2000, Jeong et 
al determined that the core metabolic networks of 43 organisms from all 3 domains of life (eukaryotes, bacteria, and archaea) displayed both scale-free and small-world architectures, ${ }^{96}$ and in 2001 they demonstrated that the protein-protein interaction network of yeast was also scalefree. ${ }^{95}$ Since then, many other biological networks have been shown to be scale-free and display small-world properties. $^{94,97-99}$

It has been postulated that the scale-free architecture of biological networks is important to the survival of cellular life, and explains the robustness of cellular systems towards random attacks. $^{31}$ If a node is attacked or fails at random in a scale-free network, probabilistically it is most likely that the node has a low connectivity, and the attack or failure will only affect the few nodes directly connected to or near that particular node. Communication amongst the majority of the network will not be interrupted by the attack/failure. This provides biological networks with a high degree of tolerance towards random attacks or node failures. ${ }^{95}$ While this architecture imparts robustness to cellular networks, it can also be exploited as a weakness by targeted or intelligent attacks. The targeted attack of highly connected "hub" nodes can be massively destructive to scale-free networks. ${ }^{92,95,100}$

This relationship between network structure and robustness demonstrates the importance in understanding the networked relationships of complex systems, which can also apply to cellular signal transduction networks. Understanding the topological characteristics of signal transduction networks is key to understanding how cells respond to their environment, and can provide a better understanding of diseased states. This can provide valuable insight for the development of effective therapeutics as well as a better understanding of the toxic effects of both pharmaceutical and environmental xenobiotics. 
A few studies have sought to determine the architecture of cancer signaling networks. ${ }^{101,}$ 102 Schramm et al analyzed the network characteristics of eleven different tumor types and corresponding non-malignant tissue samples, and found that both tumor, and normal signaling networks display scale-free characteristics. ${ }^{101}$ They found that the cancer cells had shorter average path lengths, were less centralized, were less reliant on hub proteins, and were more robust against targeted hub attacks in comparison to the non-malignant samples. These differences likely explain why cancer is difficult to treat using single target therapies. They and others also found that cancer specific mutations occur most often at hub proteins. ${ }^{101,102}$ It is important to note that the networks constructed in these studies were all based on correlations in gene expression. To fully capture the characteristics of signal transduction networks, post translational modification events, specifically phosphorylation, should be incorporated into the analysis. Ideally network analyses would be based on the directional transfer of phosphate signals through the network, but it is not currently possible to actually detect or observe the numerous interactions of proteins and transfers of phosphate groups occurring in living biological systems. This body of work presents an approach that extracts information pertaining to the networked relationships of kinases based on condition-specific protein phosphorylation data.

There are other major limitations and obstacles hindering a complete and predictive understanding of cellular networks. Most graph theory applications to biological networks fail to provide direct representations of actual cellular networks. This is because the vast majority of studies investigating biological networks rely on databases for determining the network structures. This is problematic because the databases cull data from experiments performed using a variety of different species, cell types, and experimental conditions, and therefore, 
represent more of an averaged network structure across various studies. In reality, biological networks are extremely diverse and require data-driven models for accurate depictions.

An ultimate goal of experimental and theoretical biology is to be able to understand and predict how cells will respond changes in their environment. This goal requires an intimate understanding of the structure and dynamics of the network of biological molecules that together make up the cell. Real biological networks are extremely dynamic with nodes and edges that can be added, deleted, or altered depending on the specific circumstances. However, most studies only determine the static topological structure of biological networks. Studies that only depict the static structure of biological networks fail to capture the dynamics of the processes that occur on these network structures. Characterizing dynamic processes occurring on network structures remains a challenge to network analyses across all fields. Most graph theory network analyses are based on qualitative descriptions of network connectivity. The next frontier and challenge is to make these analyses quantitative by integrating experimental data into the graph theory analysis and placing it onto the networks to capture the network dynamics. ${ }^{86,88}$ The use of weighted edges is relatively uncommon in most graph theoretical analyses, including biological network analyses, even though it is useful in describing network dynamics based on experimental data. $^{75}$ This body of work concerns the development and application of a novel graph theoretical network analysis approach that aims to address some of the obstacles currently facing biological network analyses. Importantly, this approach integrates experimentally obtained data with graph theory analysis using weighted edges, providing a means to capture the specific dynamic changes that signaling networks undergo in response to specific environmental stresses. 


\subsection{Bibliography}

(1) Lodish, H., Berk, A., Zipursky, S. L., Matsudaira, P., Baltimore, D., and Darnell, J. (2000) Isolation and Analysis of Mutants, In Molecular Cell Biology, W. H. Freeman, New York.

(2) Alon, U. (2003) Biological networks: the tinkerer as an engineer. Science, 301, 1866-1867.

(3) Bhalla, U. S., and lyengar, R. (1999) Emergent properties of networks of biological signaling pathways. Science, 283, 381-387.

(4) Carmichael, N., Bausen, M., Boobis, A. R., Cohen, S. M., Embry, M., Fruijtier-Polloth, C., Greim, H., Lewis, R., Bette Meek, M. E., Mellor, H., Vickers, C., and Doe, J. (2011) Using mode of action information to improve regulatory decision-making: an ECETOC/ILSI RF/HESI workshop overview. Crit Rev Toxicol, 41, 175-186.

(5) Pleil, J. D., and Sheldon, L. S. (2011) Adapting concepts from systems biology to develop systems exposure event networks for exposure science research. Biomarkers, 16, 99-105.

(6) Zhao, S., and lyengar, R. (2012) Systems pharmacology: network analysis to identify multiscale mechanisms of drug action. Annu Rev Pharmacol Toxicol, 52, 505-521.

(7) Downward, J. (2001) The ins and outs of signalling. Nature, 411, 759-762.

(8) Shchemelinin, I., Sefc, L., and Necas, E. (2006) Protein kinases, their function and implication in cancer and other diseases. Folia Biol (Praha), 52, 81-100.

(9) Vene, R., and Tosetti, F. (2010) The role of glycogen synthase kinase-3 in the decision between cell survival and cell death. Emerging Signaling Pathways in Tumor Biology, 95-116.

(10) Markevich, N. I., Hoek, J. B., and Kholodenko, B. N. (2004) Signaling switches and bistability arising from multisite phosphorylation in protein kinase cascades. J Cell Biol, 164, 353-359.

(11) Kemp, B. E., and Pearson, R. B. (1990) Protein kinase recognition sequence motifs. Trends in Biochemical Sciences, 15, 342-346. 
(12) Manning, B. D., and Cantley, L. C. (2002) Hitting the target: emerging technologies in the search for kinase substrates. Sci STKE, 2002, 49.

(13) Manning, G., Whyte, D. B., Martinez, R., Hunter, T., and Sudarsanam, S. (2002) The protein kinase complement of the human genome. Science, 298, 1912-1934.

(14) Endicott, J. A., Noble, M. E., and Johnson, L. N. (2012) The structural basis for control of eukaryotic protein kinases. Annu Rev Biochem, 81, 587-613.

(15) Huse, M., and Kuriyan, J. (2002) The conformational plasticity of protein kinases. Cell, 109, 275282.

(16) Piggee, C. (2009) Phosphoproteomics: miles to go before it's routine. Anal Chem, 81, 2418-2420.

(17) Ubersax, J. A., and Ferrell, J. E., Jr. (2007) Mechanisms of specificity in protein phosphorylation. Nat Rev Mol Cell Biol, 8, 530-541.

(18) Berg, J. M., Tymoczko, J. L., and Stryer, L. (2002) Covalent Modification Is a Means of Regulating Enzyme Activity, In Biochemistry, W H Freeman, New York.

(19) Yang, S. H., Sharrocks, A. D., and Whitmarsh, A. J. (2013) MAP kinase signalling cascades and transcriptional regulation. Gene, 513, 1-13.

(20) Lawrence, M. C., Jivan, A., Shao, C., Duan, L., Goad, D., Zaganjor, E., Osborne, J., McGlynn, K., Stippec, S., Earnest, S., Chen, W., and Cobb, M. H. (2008) The roles of MAPKs in disease. Cell Res, $18,436-442$.

(21) Widmann, C., Gibson, S., Jarpe, M. B., and Johnson, G. L. (1999) Mitogen-activated protein kinase: conservation of a three-kinase module from yeast to human. Physiol Rev, 79, 143-180.

(22) Krishna, M., and Narang, H. (2008) The complexity of mitogen-activated protein kinases (MAPKs) made simple. Cell Mol Life Sci, 65, 3525-3544.

(23) Morrison, D. K., and Davis, R. J. (2003) Regulation of MAP kinase signaling modules by scaffold proteins in mammals. Annu Rev Cell Dev Biol, 19, 91-118. 
(24) Sridhar, R., Hanson-Painton, O., and Cooper, D. R. (2000) Protein kinases as therapeutic targets. Pharm Res, 17, 1345-1353.

(25) Sebolt-Leopold, J. S., and English, J. M. (2006) Mechanisms of drug inhibition of signalling molecules. Nature, 441, 457-462.

(26) Azzaoui, K., Hamon, J., Faller, B., Whitebread, S., Jacoby, E., Bender, A., Jenkins, J. L., and Urban, L. (2007) Modeling promiscuity based on in vitro safety pharmacology profiling data. Med Chem, $2,874-880$.

(27) Ainsworth, C. (2011) Networking for new drugs. Nat Med, 17, 1166-1168.

(28) Jorgensen, C., and Linding, R. (2010) Simplistic pathways or complex networks? Curr Opin Genet Dev, 20, 15-22.

(29) Mellor, H. R., Bell, A. R., Valentin, J. P., and Roberts, R. R. (2011) Cardiotoxicity associated with targeting kinase pathways in cancer. Toxicol Sci, 120, 14-32.

(30) Araujo, R. P., Liotta, L. A., and Petricoin, E. F. (2007) Proteins, drug targets and the mechanisms they control: the simple truth about complex networks. Nat Rev Drug Discov, 6, 871-880.

(31) Albert, R., Jeong, H., and Barabasi, A.-L. (2000) Error and attack tolerance of complex networks. Nature, 406, 378-382.

(32) Barabasi, Albert-Laszlo, Gulbahce, Natali, Loscalzo, and Joseph. (2011) Network medicine: a network-based approach to human disease. Nat Rev Genet, 12, 56-68.

(33) Pujol, A., Mosca, R., Farres, J., and Aloy, P. (2010) Unveiling the role of network and systems biology in drug discovery. Trends Pharmacol Sci, 31, 115-123.

(34) Hopkins, A. L. (2008) Network pharmacology: the next paradigm in drug discovery. Nat Chem Biol, 4, 682-690.

(35) Hopkins, A. L. (2007) Network pharmacology. Nat Biotechnol, 25, 1110-1111. 
(36) del Sol, A., Balling, R., Hood, L., and Galas, D. (2010) Diseases as network perturbations. Curr Opin Biotechnol, 21, 566-571.

(37) Ray, L. B., and Gough, N. R. (2002) Focus Issue: The Kinome--Techniques and Methods for Analysis. Sci STKE, 162, 1-3.

(38) Xue, L., and Tao, A. (2013) Current technologies to identify protein kinase substrates in high throughput. Front Biol, 8, 216-227.

(39) Song, H., Hanlon, N., Brown, N. R., Noble, M. E., Johnson, L. N., and Barford, D. (2001) Phosphoprotein-protein interactions revealed by the crystal structure of kinase-associated phosphatase in complex with phosphoCDK2. Mol Cell, 7, 615-626.

(40) Wu, G. (2010) Functional Assays for Protein Kinases, In Assay Development pp 181-212, John Wiley \& Sons, Inc.

(41) Johnson, S. A., and Hunter, T. (2005) Kinomics: methods for deciphering the kinome. Nat Methods, 2, 17-25.

(42) Sopko, R., and Andrews, B. J. (2008) Linking the kinome and phosphorylome--a comprehensive review of approaches to find kinase targets. Mol Biosyst, 4, 920-933.

(43) Schmelzle, K., and White, F. M. (2006) Phosphoproteomic approaches to elucidate cellular signaling networks. Curr Opin Biotechnol, 17, 406-414.

(44) Houser, B. (2012) Bio-Rad's Bio-Plex suspension array system, xMAP technology overview. Arch Physiol Biochem, 118, 192-196.

(45) Khan, I. H., Mendoza, S., Rhyne, P., Ziman, M., Tuscano, J., Eisinger, D., Kung, H. J., and Luciw, P. A. (2006) Multiplex analysis of intracellular signaling pathways in lymphoid cells by microbead suspension arrays. Mol Cell Proteomics, 5, 758-768.

(46) Vignali, D. A. (2000) Multiplexed particle-based flow cytometric assays. J Immunol Methods, 243, 243-255. 
(47) Kettman, J. R., Davies, T., Chandler, D., Oliver, K. G., and Fulton, R. J. (1998) Classification and properties of 64 multiplexed microsphere sets. Cytometry, 33, 234-243.

(48) Ray, C. A., Bowsher, R. R., Smith, W. C., Devanarayan, V., Willey, M. B., Brandt, J. T., and Dean, R. A. (2005) Development, validation, and implementation of a multiplex immunoassay for the simultaneous determination of five cytokines in human serum. J Pharm Biomed Anal, 36, 10371044.

(49) Staples, E., Ingram, R. J., Atherton, J. C., and Robinson, K. (2013) Optimising the quantification of cytokines present at low concentrations in small human mucosal tissue samples using Luminex assays. J Immunol Methods, 394, 1-9.

(50) de Nadal, E., Ammerer, G., and Posas, F. (2011) Controlling gene expression in response to stress. Nat Rev Genet, 12, 833-845.

(51) Ma, Q. (2010) Transcriptional responses to oxidative stress: pathological and toxicological implications. Pharmacol Ther, 125, 376-393.

(52) Lopez-Maury, L., Marguerat, S., and Bahler, J. (2008) Tuning gene expression to changing environments: from rapid responses to evolutionary adaptation. Nat Rev Genet, 9, 583-593.

(53) Murray, J. I., Whitfield, M. L., Trinklein, N. D., Myers, R. M., Brown, P. O., and Botstein, D. (2004) Diverse and specific gene expression responses to stresses in cultured human cells. Mol Biol Cell, $15,2361-2374$.

(54) Smart, R. C., and Hodgson, E. (2013) Molecular and Biochemical Toxicology. 4 ed., John Wiley \& Sons.

(55) Kultz, D. (2005) Molecular and evolutionary basis of the cellular stress response. Annu Rev Physiol, 67, 225-257.

(56) Cowan, K. J., and Storey, K. B. (2003) Mitogen-activated protein kinases: new signaling pathways functioning in cellular responses to environmental stress. J Exp Biol, 206, 1107-1115. 
(57) Matsuzawa, A., and Ichijo, H. (2005) Stress-responsive protein kinases in redox-regulated apoptosis signaling. Antioxid Redox Signal, 7, 472-481.

(58) Fulda, S., Gorman, A., Hori, O., and Samali, A. (2010) Cellular stress responses: cell survival and cell death. Int J Cell Biol, 2010, 214074.

(59) Bhattacharya, S., Zhang, Q., Carmichael, P. L., Boekelheide, K., and Andersen, M. E. (2011) Toxicity testing in the 21st century: defining new risk assessment approaches based on perturbation of intracellular toxicity pathways. PLoS One, 6, e20887.

(60) Cargnello, M., and Roux, P. P. (2011) Activation and Function of the MAPKs and Their Substrates, the MAPK-Activated Protein Kinases. Microbiol Mol Biol Rev, 75, 50-83.

(61) Asur, R., Balasubramaniam, M., Marples, B., Thomas, R. A., and Tucker, J. D. (2010) Bystander effects induced by chemicals and ionizing radiation: evaluation of changes in gene expression of downstream MAPK targets. Mutagenesis, 25, 271-279.

(62) Roux, P., and Blenis, J. (2004) ERK and p38 MAPK-Activated Kinases: a Family of Protein Kinases with Diverse Biological Functions. Microbiol Mol Biol Rev, 68, 320-344.

(63) Benhar, M., Engelberg, D., and Levitzki, A. (2002) ROS, stress-activated kinases and stress signaling in cancer. EMBO Rep, 3, 420-425.

(64) Owuor, E. D., and Kong, A. N. (2002) Antioxidants and oxidants regulated signal transduction pathways. Biochem Pharmacol, 64, 765-770.

(65) Chen, Z., Gibson, T. B., Robinson, F., Silvestro, L., Pearson, G., Xu, B., Wright, A., Vanderbilt, C., and Cobb, M. H. (2001) MAP kinases. Chem Rev, 101, 2449-2476.

(66) Bogoyevitch, M. A. (2000) Signalling via stress-activated mitogen-activated protein kianses in the cardiovascular system. Cardiovasc Res, 45, 826-842. 
(67) Choy, G., Liu, J. W., Chandra, D., and Tang, D. G. (2005) Cell survival signaling during apoptosis: implications in drug resistance and anti-cancer therapeutic development. Prog Drug Res, 63, $115-145$.

(68) Chang, L., and Karin, M. (2001) Mammalian MAP kinase signalling cascades. Nature, 410, 37-40.

(69) Xia, Z., Dickens, M., Raingeaud, J., Davis, R. J., and Greenberg, M. E. (1995) Opposing effects of ERK and JNK-p38 MAP kinases on apoptosis. Science, 270, 1326-1331.

(70) Luscombe, N. M., Babu, M. M., Yu, H., Snyder, M., Teichmann, S. A., and Gerstein, M. (2004) Genomic analysis of regulatory network dynamics reveals large topological changes. Nature, $431,308-312$.

(71) Strogatz, S. H. (2001) Exploring complex networks. Nature, 410, 268-276.

(72) Barabási, A.-L., and Albert, R. (1999) Emergence of Scaling in Random Networks. Science, 286, 509-512.

(73) Euler, L. (1741) Solutio Problematis ad Geometriam Situs Pertinentis, Commentarii Academiae Scientiarum Imperialis Petropolitanae. Opera Omnia, 8, 128-140.

(74) Alexanderson, G. (2006) Euler and Konigsberg's bridges: A historical view. B Am Math Soc, 43, 567-573.

(75) Boccaletti, S., Latora, V., Moreno, Y., Chavez, M., and Hwang, D. U. (2006) Complex networks: Structure and dynamics. Phys Rep, 424, 175-308.

(76) Bollobas, B. (1979) Graph Theory: An Introductory Course. Springer-Verlag, NY.

(77) Albert, R., and Barabasi, A.-L. (2002) Statistical mechanics of complex networks. Rev Mod Phys, 74, 47-97.

(78) Newman, M. E. J. (2003) The Structure and Function of Complex Networks. SIAM Rev, 45, 167256. 
(79) Christensen, C., and Albert, R. (2007) Using Graph Concepts to Understand the Organization of Complex Systems. Int J Bifurcation Chaos, 17, 2201-2214.

(80) Barrat, A., Barthelemy, M., Pastor-Satorras, R., and Vespignani, A. (2004) The architecture of complex weighted networks. Proc Natl Acad Sci USA, 101, 3747-3752.

(81) Erdos, P., and Renyi, A. (1960) On the Evolution of Random Graphs. Publ Math Inst Hung Acad Sci, 5, 17-61.

(82) Alberich, R., Miro-Julia, J., and Rossello, F. (2002) Marvel Universe looks almost like a real social network. Condensed Matter, eprint arXiv:cond-mat/0202174.

(83) Tjaden, B., and Wasson, G. (2000) The Oracle of Bacon. http://oracleofbacon.org/ack.php

(84) Watts, D. J., and Strogatz, S. H. (1998) Collective dynamics of 'small-world' networks. Nature, $393,440-442$.

(85) Eisenberg, D., Marcotte, E. M., Xenarios, I., and Yeates, T. O. (2000) Protein function in the postgenomic era. Nature, 405, 823-826.

(86) Ma'ayan, A., Blitzer, R. D., and lyengar, R. (2005) Toward predictive models of mammalian cells. Annu Rev Biophys Biomol Struct, 34, 319-349.

(87) Pavlopoulos, G., Secrier, M., Moschopoulos, C., Soldatos, T., Kossida, S., Aerts, J., Schneider, R., and Bagos, P. (2011) Using graph theory to analyze biological networks. BioData Min., 4, 10.

(88) White, F. M. (2008) Quantitative phosphoproteomic analysis of signaling network dynamics. Curr Opin Biotechnol, 19, 404-409.

(89) Huber, W., Carey, V. J., Long, L., Falcon, S., and Gentleman, R. (2007) Graphs in molecular biology. BMC Bioinf, 8, s8.

(90) Aittokallio, T., and Schwikowski, B. (2006) Graph-based methods for analysing networks in cell biology. Brief Bioinform, 7, 243-255. 
(91) Grindrod, P., and Kibble, M. (2004) Review of uses of network and graph theory concepts within proteomics. Expert Rev Proteomics, 1, 229-238.

(92) Barabási, A.-L. (2009) Scale-Free Networks: A Decade and Beyond. Science, 325, 412-413.

(93) Ma'ayan, A. (2009) Insights into the Organization of Biochemical Regulatory Networks Using Graph Theory Analyses. J Biol Chem, 284, 5451-5455.

(94) Albert, R. (2005) Scale-free networks in cell biology. J Cell Sci, 118, 4947-4957.

(95) Jeong, H., Mason, S. P., Barab, A.-L., [aacute], si, and Oltvai, Z. N. (2001) Lethality and centrality in protein networks. Nature, 411, 41-42.

(96) Jeong, H., Tombor, B., Albert, R., Oltvai, Z. N., and Barabasi, A. L. (2000) The large-scale organization of metabolic networks. Nature, 407, 651-654.

(97) Barabasi, A. L., and Oltvai, Z. N. (2004) Network biology: understanding the cell's functional organization. Nat Rev Genet, 5, 101-113.

(98) Han, J.-D. J., Bertin, N., Hao, T., Goldberg, D. S., Berriz, G. F., Zhang, L. V., Dupuy, D., Walhout, A. J. M., Cusick, M. E., Roth, F. P., and Vidal, M. (2004) Evidence for dynamically organized modularity in the yeast protein-protein interaction network. Nature, 430, 88-93.

(99) Giot, L., Bader, J. S., Brouwer, C., Chaudhuri, A., Kuang, B., Li, Y., Hao, Y. L., Ooi, C. E., Godwin, B., Vitols, E., Vijayadamodar, G., Pochart, P., Machineni, H., Welsh, M., Kong, Y., Zerhusen, B., Malcolm, R., Varrone, Z., Collis, A., Minto, M., Burgess, S., McDaniel, L., Stimpson, E., Spriggs, F., Williams, J., Neurath, K., loime, N., Agee, M., Voss, E., Furtak, K., Renzulli, R., Aanensen, N., Carrolla, S., Bickelhaupt, E., Lazovatsky, Y., DaSilva, A., Zhong, J., Stanyon, C. A., Finley, R. L., Jr., White, K. P., Braverman, M., Jarvie, T., Gold, S., Leach, M., Knight, J., Shimkets, R. A., McKenna, M. P., Chant, J., and Rothberg, J. M. (2003) A protein interaction map of Drosophila melanogaster. Science, 302, 1727-1736.

(100) Motter, A. (2004) Cascade Control and Defense in Complex Networks. Phys Rev Lett, 93, 1-4. 
(101) Schramm, G., Kannabiran, N., and König, R. (2010) Regulation patterns in signaling networks of cancer. BMC Syst Biol, 4, 162.

(102) Cui, Q., Ma, Y., Jaramillo, M., Bari, H., Awan, A., Yang, S., Zhang, S., Liu, L., Lu, M., O'ConnorMcCourt, M., Purisima, E. O., and Wang, E. (2007) A map of human cancer signaling. Mol Syst Biol, 3, 152. 


\section{Chapter 2}

Network Analysis Approach to Determine Xenobiotic Toxic Modes of Action 


\subsection{Introduction}

Our understanding of cellular functioning has increased significantly over the past few decades. As our view of the cell and intracellular signaling expands, it becomes more and more difficult to delineate how all of the individual cellular components engage to form the sophisticated communication systems that coordinate biological processes. The architecture of signaling networks inherently influences their function. ${ }^{l}$ Current toxicological mode of action (MOA) evaluations seldom consider network relevant information, even though xenobiotic effects are ultimately guided by the response of the signaling network. In this chapter, a novel graph theoretical approach to analyze networked biological responses to toxicological insult is presented. The approach is utilized to provide a network relevant description of a xenobiotic MOA. Importantly, this approach integrates experimentally obtained data with graph theory analysis, providing a means to capture the specific dynamic changes that signaling networks undergo in response to specific xenobiotic exposures.

\subsubsection{Mode of Action Analyses}

An important component of human toxicological risk assessments is identification of a compounds MOA. Determining the MOA by which uncharacterized xenobiotics lead to adverse effects is currently a challenging task. Determination of just one compound's MOA requires years of effort, and there are currently tens of thousands of environmental and pharmaceutical compounds with unknown toxic MOAs..$^{2-5}$ In spite of extensive efforts, the cellular targets of many toxic xenobiotics remain unknown. ${ }^{5,6}$ This can be partially attributed to difficulties in identifying a few principal protein targets when there are thousands of other proteins functioning 
together as complex and dynamic biological signaling networks to coordinate the cell's response to toxic exposure.

The initial pharmacodynamic response to xenobiotic exposure is predominantly coordinated by signal transduction networks that follow the simple framework of protein phosphorylation and dephosphorylation carried out by kinases and phosphatases. This framework yields a complex cellular signaling network consisting of thousands of proteins, of which any can be targets in the xenobiotic toxic MOA. ${ }^{7,8}$ The vast size and dynamic nature of signaling networks makes it a challenge to identify the primary target(s) of uncharacterized xenobiotics. Though difficult in itself, identification of the primary xenobiotic target falls short of providing a complete understanding of the toxic effects of a chemical exposure. Toxic actions can and will propagate throughout cellular networks, sometimes affecting seemingly distant components. The propagation of pharmaceutical actions throughout a signaling network is sometimes responsible for undesired side effects. ${ }^{9,} 10$ It is believed that most pharmaceutical compounds interact with at least 6 targets. $^{11,12}$ Xenobiotic promiscuity can induce multiple propagations throughout the network, further complicating the determination of the toxic MOA. It is difficult to fully capture the toxic mechanism of action for an uncharacterized compound without considering the networked nature of the cell. Approaches that can accurately and efficiently describe xenobiotic MOAs from a network perspective have the potential to save the pharmaceutical industry a substantial amount of time and money while leading to the development of safer therapeutics. ${ }^{10}$

Current methods of determining toxic MOAs involve years of testing single compounds, primarily utilizing long term whole animal studies and a battery of in vitro assays. ${ }^{13-16}$ The data obtained from these extensively studied compounds is then incorporated into large reference 
libraries. The MOA for other compounds can then be predicted in a shorter time frame with fewer resources by generating an activity profile for the compound using just a few assays and comparing the results to those in the reference library. ${ }^{17-22}$ These activity profiling approaches still require a considerable amount of time and resources, and are limited to identifying MOAs that are contained within the reference library. Such approaches are not well suited for evaluating unique compounds that do not exhibit activities similar to those of well studied compounds.

Quantitative structure activity relationship (QSAR) information is also used in MOA analyses to identify potential xenobiotic targets. QSAR approaches predict targets by comparing the structural properties of the studied xenobiotic to reference compounds with known protein targets and activities. ${ }^{21,23-27}$ Compounds can also be reacted with large reference panels of isolated proteins to identify potential target interactions as part of MOA analysis. ${ }^{12}$ However, simply identifying the proteins possibly bound by a molecule does not effectively capture the network wide effects of the compound, nor describe how these effects contribute to the progression towards toxicity. Additionally, isolated proteins do not accurately reflect the native environment of proteins within signaling networks, as toxicity can be an emergent property of the network and its structure. Also, there is always a chance that the target for which the compound has the highest affinity is not in the reference set. ${ }^{28,29}$

While these currently used methods of evaluating xenobiotic MOAs are informative, there is a need for more efficient and more network comprehensive complimentary approaches that are capable of rapidly characterizing a specific biological systems' response to xenobiotic exposure (based only on data from that system), while identifying key proteins involved in the MOA. This chapter demonstrates the ability of a newly developed network analysis approach to 
efficiently identify primary xenobiotic targets and other proteins important to a xenobiotic toxic MOA. As a proof of principle, the approach's ability to assess a MOA is tested using a xenobiotic known to selectively inhibit glycogen synthase kinase 3 (GSK-3).

\subsubsection{GSK-3 and TDZD-8}

GSK-3 is a multifunctional protein involved in the regulation of a diverse array of

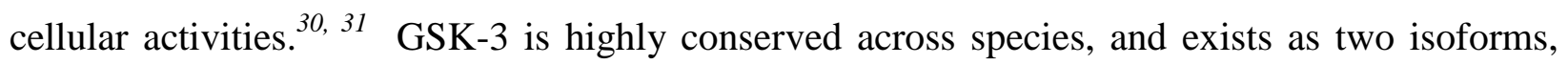
GSK-3 $\alpha$ and GSK-3ß. Both isoforms are expressed ubiquitously in tissue. ${ }^{32}$ GSK-3 has received significant attention as a therapeutic target due to its involvement in a variety of pathological conditions including type II diabetes, muscle wasting, cancer, neurological disorders, inflammation, and Alzheimer's. ${ }^{33}$ It was originally discovered as a protein involved in glycogen metabolism, but is now seen as a complex nexus protein capable of interacting with many different substrates and multiple signaling pathways. ${ }^{32}$ Few enzymes exert more influence over cellular function than GSK-3, and GSK-3 is one of the kinases with the greatest number of identified cellular substrates. ${ }^{34}$ There are multiple mechanisms by which GSK-3 activity is regulated including phosphorylation and autophosphorylation, protein complex association, substrate priming, subcellular localization, and proteolytic cleavage. ${ }^{30,}{ }^{32}$ While all of the regulatory mechanisms for GSK-3 are not fully understood, GSK-3 is known as a dual specificity kinase that is differentially regulated by Tyr and Ser/Thr phosphorylation ${ }^{32}$. GSK-3 is positively regulated by phosphorylation on a Tyr residue (Tyr-279 on GSK-3 $\alpha$ and Tyr-216 on GSK-3ß), and negatively regulated by N-terminal phosphorylation of Ser residues (Ser-21 on GSK-3 $\alpha$ and Ser-9 on GSK-3ß). ${ }^{32,35,36}$ The Ser residues can be phosphorylated by several protein kinases including AKT, p90RSK, and p70S6 kinase. ${ }^{32}$ An uncommon feature of GSK-3 
is that it is catalytically active in the absence of extracellular stimulation, but in response to extracellular signals its enzymatic activity rapidly decreases. ${ }^{37}$

Over 100 cytoplasmic and nuclear proteins have been identified as GSK-3 substrates. ${ }^{32}$ A rare feature of GSK-3 is that many (but not all) of GSK-3's substrates must first be primed by phosphorylation before GSK-3 will act on them. ${ }^{37,38}$ These substrates span various functional types of proteins including those involved in metabolism, cellular architecture, gene expression, neurobiological processes, immune responses, circadian rhythms, differentiation, proliferation, growth, insulin response, death and survival. ${ }^{32,33}$

The multi-functionality and pivotal role that GSK-3 plays in many biological processes and diseases has led to a high level of interest in GSK-3 as a treatment option for many diseases, and the subsequent development of many GSK-3 inhibitors in the past decade. ${ }^{33}$ Many of these inhibitors are being developed for the treatment of diseases including Alzheimer's, ${ }^{39-41}$ diabetes, ${ }^{42}$ inflammatory disorders, ${ }^{42}$ and cancer. ${ }^{43}$ However, caution must also be heeded when considering GSK-3 as a therapeutic target. While GSK-3's involvement in a variety of processes provides the opportunity to treat a variety of conditions, it also provides the opportunity to interfere with a variety of processes possibly increasing the potential for undesired side effects. ${ }^{39}$ Understanding the effects of GSK-3 inhibition is difficult and depends on the specific context in which the inhibitor is used.

Due to the therapeutic interest in GSK-3 inhibition and GSK-3's complex nature, the approach developed in this body of work was utilized to assess the effects of the selective GSK$3 ß$ inhibitor, 4-Benzyl-2-methyl-1,2,4-thiadiazolidine-3,5-dione (TDZD-8). TDZD-8 was one of the first developed non-ATP competitive GSK-3ß inhibitors, and was synthesized as a potential Alzheimer's treatment. ${ }^{44}$ TDZD-8 binds to the active site of GSK-3ß inhibiting its activity 
towards glycogen synthase-1 (GS-1) with an $\mathrm{IC}_{50}$ concentration of $2 \mu \mathrm{M}^{44}$ In rat models, TDZD-8 has been shown to induce Ser-9 phosphorylation on GSK-3ß without altering Tyr-216 phosphorylation, but the precise mechanisms underlying TDZD-8's inhibitory effects are currently undetermined. $^{36}$ Figure $2-1$ shows the structure of TDZD-8.

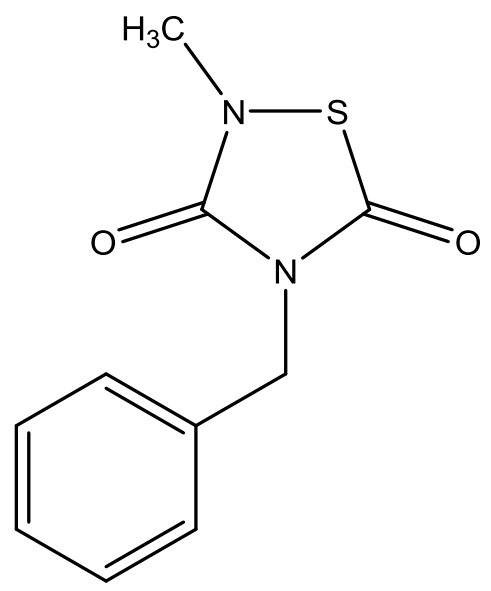

Figure 2-1. TDZD-8

\subsubsection{Approach Overview}

The approach presented in this work extracts information pertaining to the cellular network response to xenobiotics, such as TDZD-8, based on the levels of phosphorylated protein obtained using the bead-based immunoassay platform discussed in chapter 1. Euclidean distances between the relative protein phosphorylation levels of all possible protein pairs are calculated for each exposure condition, and used to describe the similarity in each pair of proteins' responses to the stimulation. Euclidean distances are simple metrics that have found previous application in describing similar responses when analyzing biological networks. ${ }^{45-47}$ The approach presented is based on the postulation that a similar level of response for proteins indicates that there exists a relatively close connection (direct or indirect) between the proteins in the network and thus, the calculated Euclidean distances can be used to represent the "distance" 
between each pair of proteins in the network. These distances are then used as weighted edges for graph theory analysis, and parameters that characterize the network are calculated.

Graph theory has proven to be a valuable mathematical modeling tool for gaining insights into the topological organization of networks across various disciplines. ${ }^{45,}$ 48-50 In graph theoretical analyses, networks are depicted as graphs where objects in the network are represented as nodes, and the pair wise relations between the objects are represented by lines referred to as edges. Based on the connectivity of the graphs, statistical attributes that characterize the structure and behavior of networked systems can be calculated. ${ }^{48-52}$ Since its development in the $18^{\text {th }}$ century, graph theory has found application in the study of many networks including social, computer, economic, and, most recently, biological networks. ${ }^{45}$, 53-59 While advances in high-throughput data collection techniques have led to a rapid increase in the application of graph theory analyses to the study of biological signaling networks, it has yet to be used in toxicology, and specifically has never been applied to dose.

A common part of many graph theory analyses, including the analysis presented here, is centrality analysis. Centrality analyses typically rank network elements using quantitative node centrality parameters to identify interesting network elements. ${ }^{58}$ Node centralities allow quantitative local measurement of the position of a node relative to other nodes in a network, and can be used to infer the relative significance of a node in global network organization. ${ }^{l}$ The equations used to calculate the many centrality parameters that exist reflect a particular node's network position in a variety of ways. The calculations commonly describe a node's connectedness with other "neighbor" nodes. A path is a series of edges and nodes in a network that can be used to connect one node to another. The determination of the shortest path connecting a node and other nodes within the network is a common component of many node 
centrality calculations. The shortest path is the shortest possible sequential series of edges that can be utilized to connect two nodes to each other. When edges are not weighted, the shortest path length is equal to the number of edges connecting two nodes. When edges are given weightings that describe the strength of the connection between nodes, the shortest path is the shortest possible summation of the edge weight values assigned to the edges connecting two nodes. High centrality scores typically indicate that a node can reach other nodes via relatively short path lengths or that a node is frequently passed through in the shortest paths connecting other nodes. ${ }^{60}$

In biological analyses, centrality parameters are most commonly used to identify proteins that are functionally important to biological processes. ${ }^{58}$ In this chapter, centrality analysis was used to identify key proteins involved in the MOA of a xenobiotic. This differs slightly in comparison to traditional biological centrality analyses in that it does not focus on identifying the most central proteins/nodes in the network, but monitors changes in the centrality of proteins to determine which proteins are affected the most by xenobiotic exposure. In this chapter, the node centrality parameter, radiality, was calculated for several intracellular proteins based on their relative phosphorylation responses to increasing doses of TDZD-8. Based on changes in radiality, the proteins most affected by the xenobiotic were identified. The developed approach maps cellular component responses based on networked relationships, providing an integrated view of the effects exerted by xenobiotic exposure, and highlighting principal proteins involved in the toxic mode of action.

\subsubsection{Radiality}

Radiality is a centrality parameter that was originally developed by Valente and Foreman in 1998 for the analysis of social networks. ${ }^{61}$ Radiality is calculated based on the relationships 
between a node and its neighbors, but also includes information pertaining to the entire network structure. $^{61}$ In general, radiality indicates how integrated an individual node is with the rest of the network. Therefore, radiality represents a suitable means to identify proteins that are most greatly affected by a particular xenobiotic exposure.

Radiality is calculated by first computing the shortest paths, represented as $\operatorname{dist}(v, \omega)$, connecting all possible pairs of proteins in the network, where $v$ and $\omega$ represent the two proteins for which the shortest path distance corresponds. The network parameter, diameter $\left(\Delta_{G}\right)$, is then determined. The diameter is the maximum value obtained for the shortest path between any two nodes in the entire network. Next, the value of each determined shortest path are subtracted from the value of the diameter plus one. The resulting values for a particular node to all other nodes in the network are then summed and divided by the total number of nodes $(n)$ in the network minus one to obtain the radiality value for that particular node. If a node has a high radiality it is generally close to all other nodes in the network ${ }^{l}$. The radiality equation is shown below.

$$
C_{\text {rad }}(v)=\frac{\sum_{\omega \in N}\left(\Delta_{G}+1-\operatorname{dist}(v, \omega)\right)}{n-1}
$$

Centrality parameters can be interpreted in various ways, depending on the specific application of the analysis. ${ }^{62}$ Radiality as calculated in this work, concisely and simultaneously describes each protein's phosphorylation-mediated relation to all other proteins in the network. Mechanistically, this means that a protein/node with low radiality is not highly integrated within the network under a set of experimental conditions. A change in a protein's radiality following xenobiotic exposure provides evidence that the protein is affected by the xenobiotic and may be a principal protein in the xenobiotics toxic MOA. In this work, radiality values were calculated using the open source software platform Cytoscape along with a specially developed version of 
the Cytoscape plug-in, CentiScape. ${ }^{63}$ The CentiScaPe plug-in was modified so that centrality parameters were calculated using the Euclidean distance values as edge weights.

TDZD-8's effects on 20 individual protein roles within the signaling network were evaluated on an individual dose basis, and dose-response curves consisting of the radiality parameter were constructed to describe changes in network connectivity. The data driven approach does not use any pre-existing knowledge of the xenobiotics structure or activity. It captures the xenobiotic effects on a network level by describing perturbations in the relationships between individual signaling proteins. The approach presented represents a complimentary approach to those currently used to determine MOAs, because it can be used to evaluate virtually any toxic compound from a network perspective in a relatively short time frame.

\subsection{Experimental Procedures}

\subsubsection{Materials}

4-Benzyl-2-methyl-1,2,4-thiadiazolidine-3,5-dione (TDZD-8, CAS 327036-89-5), Dulbecco's modified Eagle's medium (DMEM), sodium pyruvate, D-glucose, L-glutamine, and sodium bicarbonate were obtained from Sigma Aldrich (St. Louis, MO). HEPES, fetal bovine serum, Ethidium homodimer-1 cytotoxicity kit, and penicillin-streptomycin were obtained from Invitrogen (Carlsbad, CA). Human hepatocellular carcinoma-derived HepG2 cell line was obtained from American Type Culture Collection (Manassas, VA).

\subsubsection{Cell culture}

HepG2 cells were cultured in DMEM, supplemented with 2 g/L D-glucose, $2 \mathrm{mM} \mathrm{L-}$ Glutamine, $5 \mathrm{mM}$ HEPES, $24 \mathrm{mM}$ sodium bicarbonate, $1 \mathrm{mM}$ sodium pyruvate, $10 \%$ fetal bovine serum, $100 \mathrm{U} / \mathrm{mL}$ penicillin, and $100 \mathrm{mg} / \mathrm{mL}$ streptomycin. Cells were maintained in a humidified atmosphere at $37^{\circ} \mathrm{C}, 5 \% \mathrm{CO}_{2}$ and passaged at $80 \%$ confluence. 


\subsubsection{Dosing.}

For plasma membrane degradation assays, cells were seeded into clear-bottom, blacksided 96-well plates at a concentration of $4 \times 10^{4}$ cells per well in DMEM without phenol red and allowed to grow for $24 \mathrm{~h}$ before dosing. For multiplex phosphoprotein assays, cells were seeded in 12 -well plates at a concentration of $5 \times 10^{5}$ cells per well in DMEM without phenol red and allowed to grow for $24 \mathrm{~h}$. Following the $24 \mathrm{~h}$ growth period, medium was aspirated from wells, and cells were challenged with $10,20,30,40,50$, or $100 \mu \mathrm{M}$ concentrations of TDZD-8. TDZD8 was prepared so that resulting well concentrations were $<1 \%$ DMSO.

\subsubsection{Multiplex Phosphoprotein Assay}

After $40 \mathrm{~min}$ or $10 \mathrm{~h}$ of exposure to increasing doses of TDZD-8 (10, 20, 30, 40, 50, or $100 \mu \mathrm{M})$, cells were washed with ice cold PBS and lysed. Total protein concentration was determined using the DC Protein Assay (BioRad, Hercules, CA) according to the manufacturer's instructions. Protein phosphorylation was determined using the bead-based BioPlex suspension array system (Bio-Rad, Hercules, CA), and lysates were prepared according to the manufacturer's protocol. Beads and detection antibodies against phosphorylated AKT (Ser473), ERK1/2(Thr202/Tyr204, Thr185/Tyr187), GSK-3 $\alpha / \beta$ (Ser21/Ser9), HSP27 (Ser78), IкB $\alpha$ (Ser32/Ser36), JNK1/2 (Thr 183/Tyr185), MEK1 (Ser217/Ser221), p38MAPK (Thr180/Tyr182), p53 (Ser15), and p90RSK (Thr359/Ser363), as well as cleaved PARP and activated caspase-3 were obtained from Bio-Rad (Hercules, CA). Beads and detection antibodies against phosphorylated BAD (Ser 112), CREB1 (Ser133), IGFR (Tyr1131), IRS1 (Ser636/Ser639), EGFR (Tyr), MSK1(Ser212), p70S6 (Thr421/Ser424), AKT(Thr308), and p53(Ser46) were obtained from EMD Millipore. Relative phosphorylation was calculated by normalizing to 
control, which only received dosing vehicle (1\% DMSO). Experiments were performed in triplicate.

\subsubsection{Plasma Membrane Degradation Assay}

To determine the relative level of plasma membrane degradation in HepG2 cells exposed to increasing doses of TDZD-8, the ethidium homodimer-1 (EthD-1) assay was used. Twentyfour hours after seeding HepG2 cells, culture medium was removed and replaced with probecontaining culture medium ( $2 \mu \mathrm{M}$ EthD-1). Dead control wells were exposed to $70 \%$ methanol, and the plate was incubated for 30 minutes. Following the 30 minute incubation, cells were challenged with increasing doses of TDZD-8 and placed in the plate reader immediately after dosing. Fluorescent signal was obtained using the Infinite M1000 microplate reader (Tecan US, Raleigh NC) with excitation wavelength of $530 \mathrm{~nm}$ and emission wavelength of $645 \mathrm{~nm}$, read from the bottom $40 \mathrm{~min}$ and $10 \mathrm{~h}$ following dosing. Experiments were performed in sextuplicate. Relative plasma membrane degradation was determined by normalizing to dead control cells exposed to $70 \%$ methanol.

\subsubsection{Data Analysis.}

Statistical significance for relative phosphorylation and plasma membrane degradation was assessed using two-way analysis of variance (ANOVA) with Bonferroni's post-test. $p<$ 0.05 was considered statistically significant. For plasma membrane degradation and relative phosphorylation data, error bars reflect standard error of the mean (SEM). Euclidean distances (E) were determined using SAS JMP (Carey, NC) and the equation 


$$
E(v, \omega)=\sqrt{\sum_{i=1}^{n}\left(\left(\text { Relative Phosphorylation } v_{i}\right)-\left(\text { Relative Phosphorylation } \omega_{i}\right)\right)^{2}}
$$

Where $v$ and $\omega$ represent the 2 protein nodes for which the distance between is being calculated. All Euclidean distances were multiplied by 100 to facilitate network parameter calculations with CentiScape. The node centrality parameter, radiality, was calculated based on the Euclidean distance edge weights. There was no set threshold value for the existence of an edge, and thus all distance values were used in the calculation of radiality. The node centrality parameter, radiality $\left(C_{\text {rad }}(v)\right)$, is calculated using the equation

$$
C_{r a d}(v)=\frac{\sum_{\omega \in N}\left(\Delta_{G}+1-\operatorname{dist}(v, \omega)\right)}{n-1}
$$

where $\Delta_{\mathrm{G}}$ is the network diameter (or the longest Euclidean distance pathway connecting any 2 nodes in the network), $n$ is the number of nodes in the network, and $\operatorname{dist}(v, \omega)$ is the shortest possible summation of Euclidean distance weighted edge lengths connecting node $v$ to node $\omega$. $\Delta_{\mathrm{G}}$ represents the compactness of the network, and can be interpreted as a relative estimate of the ease of protein communication. When interpreting centrality from $C_{\text {rad }}(v)$, if the radiality of a node, $v$, is high, then the node is generally close to all other nodes in the network, and therefore is considered central. ${ }^{l}$

\subsection{Results}

Forty min and $10 \mathrm{~h}$ TDZD-8 exposure time points were identified as points where critical signaling events occur in a previous study by kinetically monitoring energy production processes 
in the cell, and were therefore utilized in this study. ${ }^{64}$ Relative plasma membrane degradation following exposure to increasing concentrations $(10,20,30,40,50$, and $100 \mu \mathrm{M})$ of TDZD-8 for these critical time points is shown in Figure 2-2. Two-way ANOVA comparing the relative plasma membrane degradation in cells treated with TDZD-8 to control cells showed that there were no significant differences in plasma membrane degradation following both $40 \mathrm{~min}$ and $10 \mathrm{~h}$ exposure to 10 or $20 \mu \mathrm{M}$ doses. Plasma membrane degradation increased significantly $(p<0.01)$ in cells treated with 30 and $40 \mu \mathrm{M}$ TDZD-8 for $10 \mathrm{~h}$ (compared to controls), but not in cells treated for 40 min. Plasma membrane degradation increased significantly $(p<0.001)$ in cells treated with 50 or $100 \mu \mathrm{M}$ TDZD-8 for both $40 \mathrm{~min}$ and $10 \mathrm{~h}$.

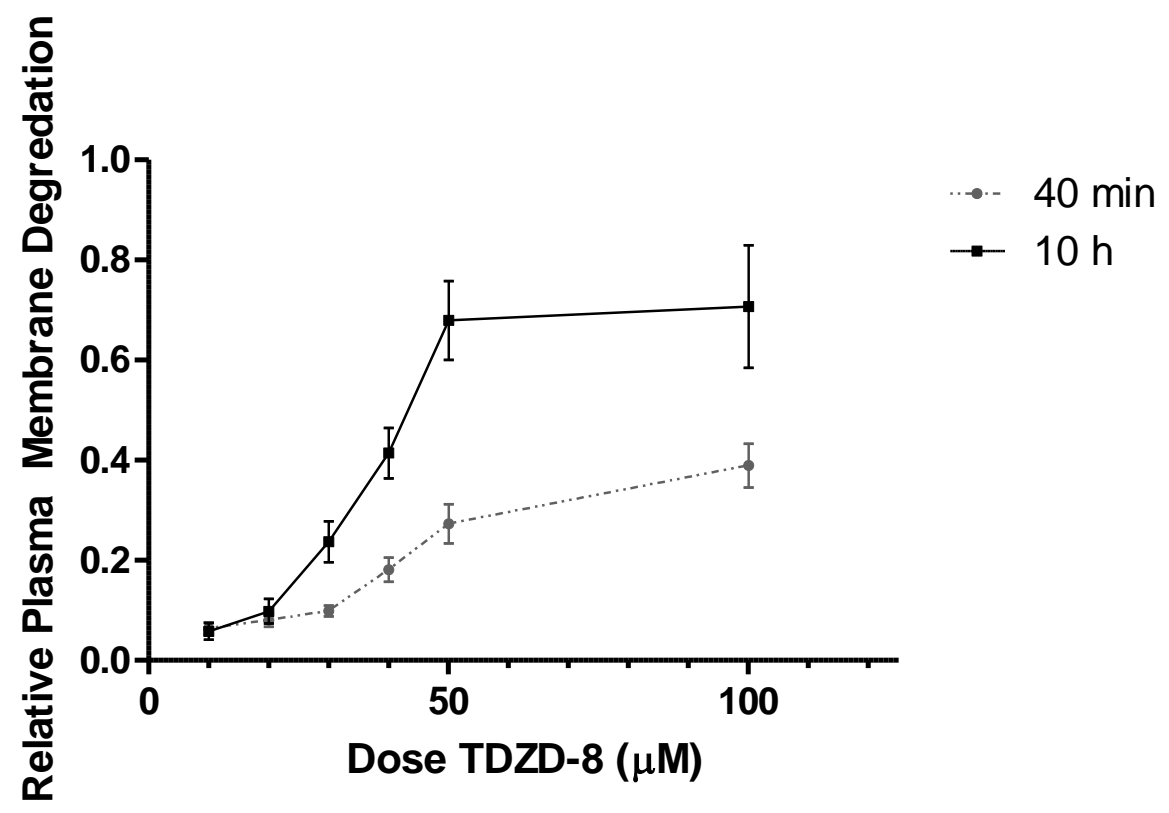

Figure 2-2. Relative plasma membrane degradation in response to exposure to increasing doses of TDZD-8 for $40 \mathrm{~min}$ and $10 \mathrm{~h}$.

Relative phosphorylation was determined for cells exposed to the same increasing concentrations of TDZD-8 for $40 \mathrm{~min}$ and $10 \mathrm{~h}$, and is depicted in Figures 2-3 and 2-4, respectively. Simple inspection of the phosphorylation data does not concisely describe the effects of TDZD-8 on the signaling network or provide a clear indication of its primary target. 


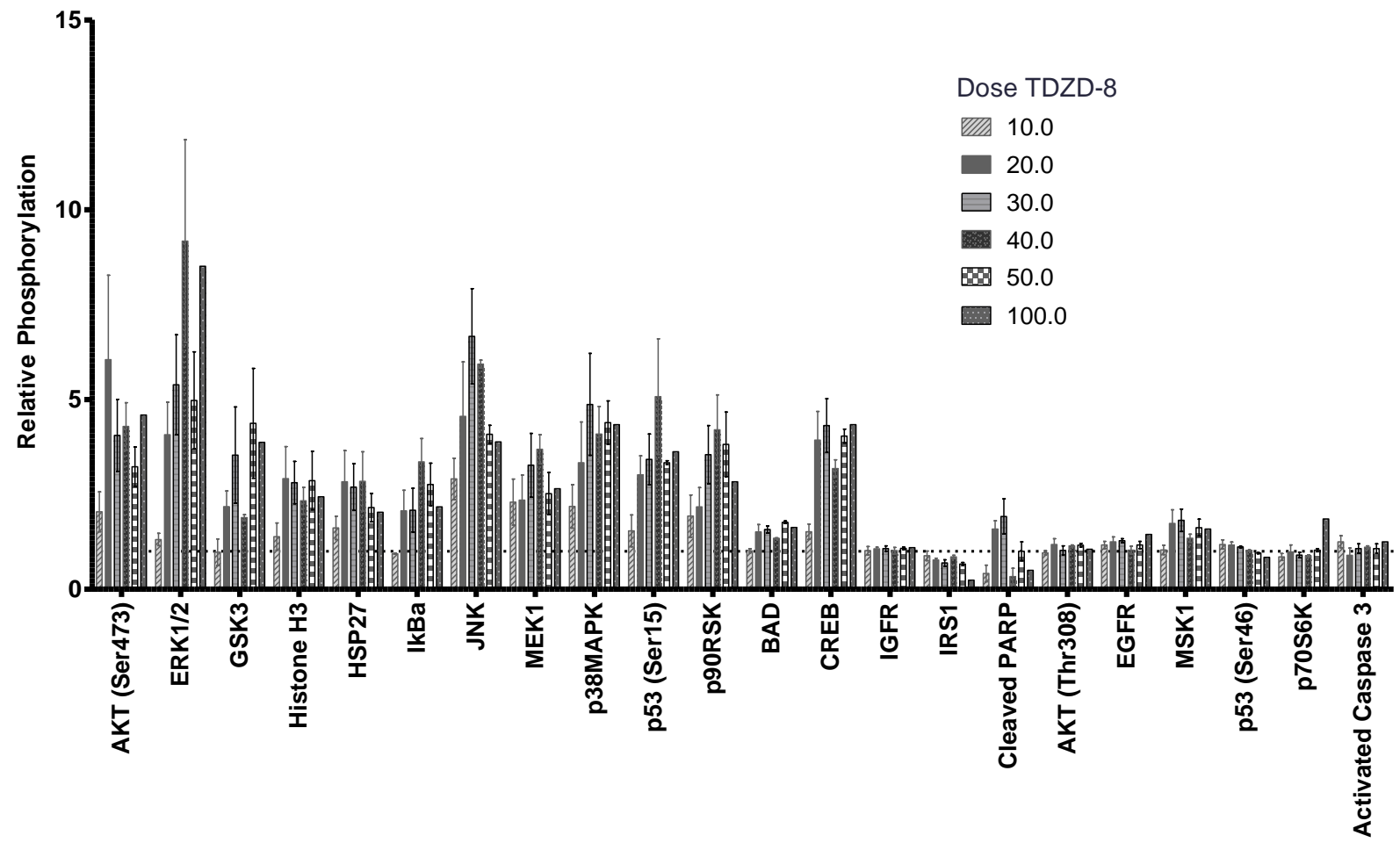

Figure 2-3. Phosphoprotein response to TDZD-8 at $40 \mathrm{~min}$. 


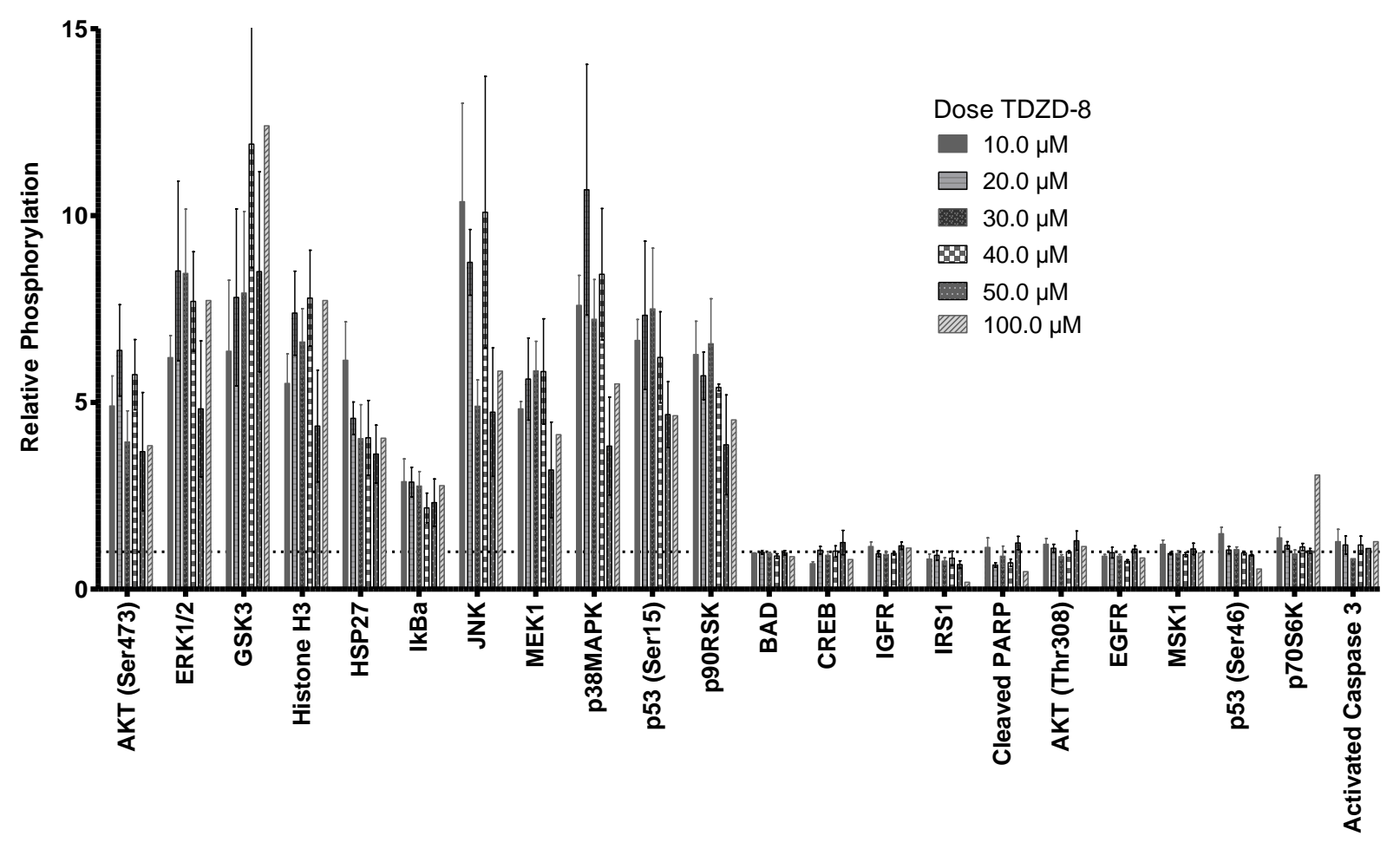

Figure 2-4. Phosphoprotein Response to TDZD-8 at $10 \mathrm{~h}$.

To describe the networked response to treatment with TDZD-8, radiality was calculated for each protein at each individual dose tested. Dose-response curves consisting of the radiality values were constructed (Figure 2-5). Fluctuations in radiality values across the dosing range indicate that the inhibitor treatment is causing the networked relationship between signaling proteins to change. A decreasing radiality value for a protein suggests it is losing communication with the majority of proteins in the network. When using graph theory centrality parameters, nodes are often ranked by their centrality values, or compared to the mean centrality value for all nodes. Following 40 min exposure to TDZD-8, AKT (Ser 473), Cleaved PARP, CREB, JNK, p38, ERK1/2, GSK-3a/ß, and p53 (Ser15) were the only proteins with radiality values that were at least one standard deviation unit less than the mean for any of the doses 
tested. CREB, ERK1/2, and JNK radiality values were at least one standard deviation unit less than the mean for 4 or more of the 6 doses tested. AKT, Cleaved PARP, p38, GSK-3, and p53 (Ser15) radiality values were only one or more standard deviation units less than the mean for one dose. There were no proteins with radiality values more than one standard deviation unit above the mean. Table 1 shows the mean radiality values for each dose and the corresponding standard deviation.

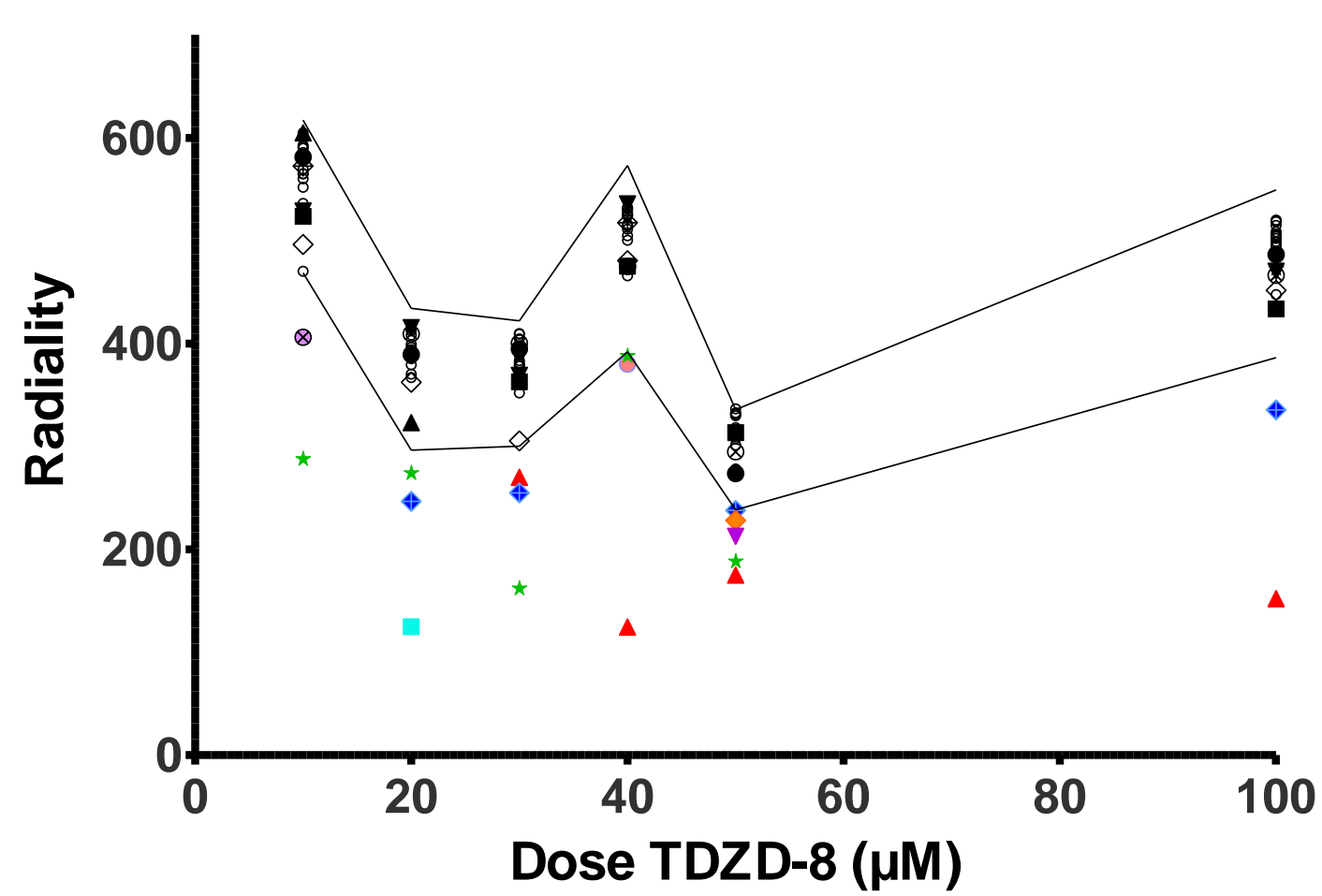

AKT (Ser473) Cleaved PARP CREB

$\triangle$ ERK $1 / 2$

$\nabla$ GSK3

$\diamond$ p38

$\star$ JNK

- p53 (Ser15)

- Mean + SD

- Mean - SD

- AKT (Thr308)

- BAD

- EGFR

- Activated Caspase 3

- Histone H3

- HSP27

- IGFR

- IkBa

- IRS1

- MEK1

- MSK1

- p53 (Ser46)

- p70s6

- p90RSK

Figure 2-5. Radiality following 40 min TDZD-8 exposure.

Table 2-1. 40 min TDZD-8 mean radiality

\begin{tabular}{rcc}
\hline Dose $(\mu \mathrm{M})$ & Mean Radiality & Standard Deviation \\
\cline { 3 - 4 } \cline { 3 - 3 } 20 & 543 & 74 \\
20 & 365 & 69 \\
30 & 361 & 61 \\
40 & 482 & 91 \\
50 & 287 & 49 \\
100 & 468 & 81 \\
\hline
\end{tabular}


Following 10 h exposure to TDZD-8, ERK1/2, Histone H3, JNK, p38, p53 (Ser15), and GSK-3 radiality values were at least one standard deviation unit less than the mean. Histone H3 radiality was only one standard deviation unit less than the mean for the $100 \mu \mathrm{M}$ dose. All of the other mentioned proteins had radiality values at least one standard deviation less than the mean for at 3 or more of the doses tested. GSK-3 radiality values reached the lowest of any proteins in the study for $10 \mathrm{~h}$ exposed cells, and GSK-3 was the only protein with radiality values more than 2 standard deviation units less than the mean. Figure 2-6 shows the radiality dose-response curves for cells exposed to TDZD-8 for $10 \mathrm{~h}$. 


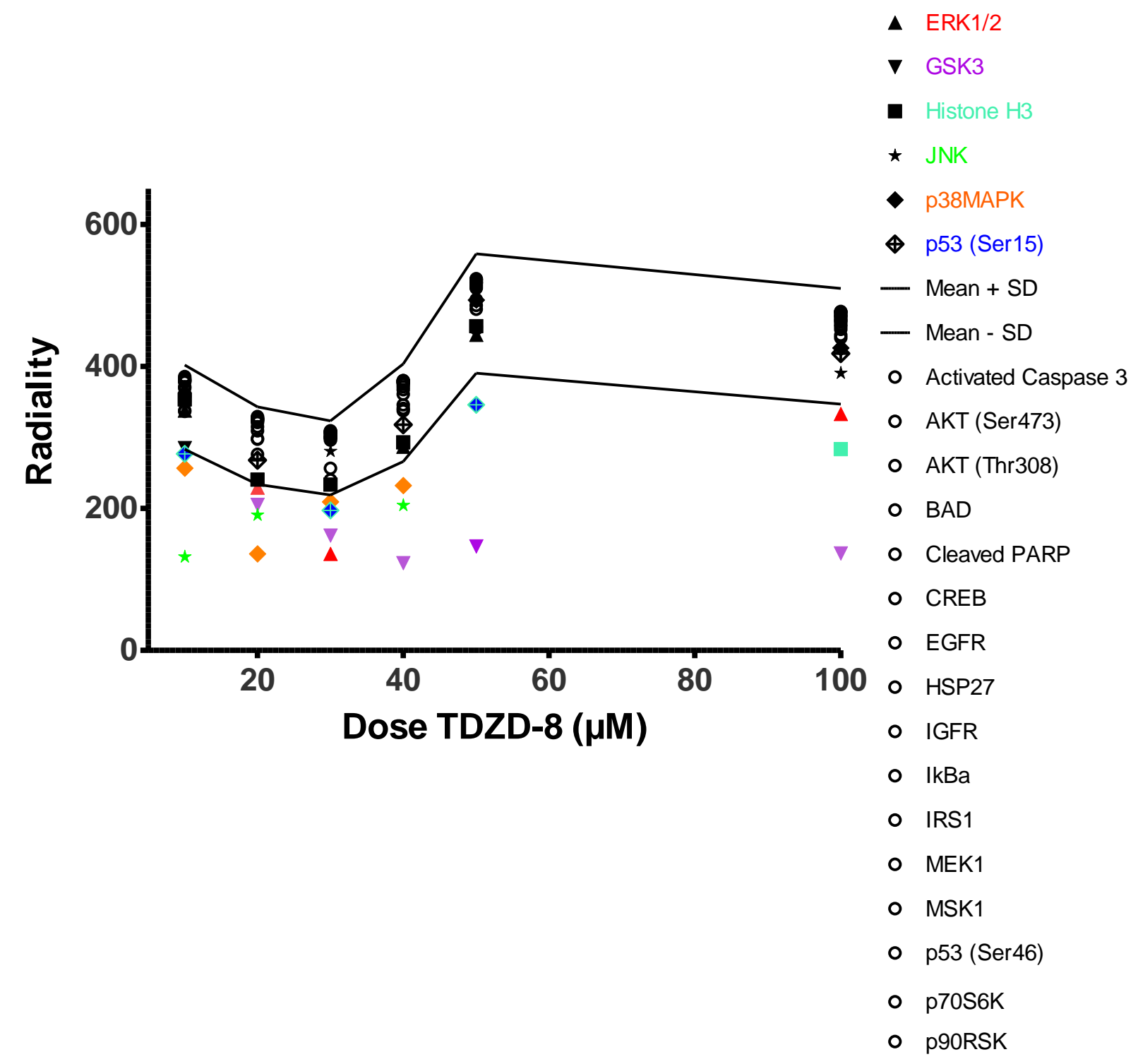

Figure 2-6. Radiality following $10 \mathrm{~h}$ TDZD-8 exposure.

\subsection{Discussion}

The identification of toxic modes/mechanisms of response to xenobiotic exposure is challenging in part due to the dynamic and complex networked nature of biological systems. This is confounded by the fact that the existing interconnected relationships between the numerous components that form biological network structures are poorly understood even in normal undisturbed physiological states. There is a current need for approaches that are capable 
of rapidly characterizing a broad range of toxic mechanisms of response to virtually any compound so that large numbers of compounds can be easily screened, and their potential impact on human health may be assessed for both environmental and pharmaceutical purposes.

The developed graph theoretical approach was used to map cellular component responses to toxic GSK-3 inhibition, and identify the principal proteins involved in the response mechanism. By monitoring the centrality (radiality) of each protein in response to increasing doses of inhibitor, key proteins whose role within the network changed, and are therefore likely to be involved in the MOA, were identified. The obtained dose-response curves clearly indicated GSK-3 as the primary target of TDZD-8 following $10 \mathrm{~h}$ exposure. In addition to identification of GSK-3 as the primary target of the inhibitor, perturbations in the network roles of several other signaling proteins were identified, indicating their possible involvement in the toxic MOA. Following exposure to TDZD-8 for 40 min, AKT (Ser 473), cleaved PARP, CREB, JNK, p38, ERK1/2, GSK-3, and p53 (Ser 15) were identified as proteins whose radiality was most greatly affected. This agrees with research studies that have found GSK-3 inhibition to alter the activity of CREB, p38, ERK1/2, JNK, p53, PARP, and AKT. ${ }^{31,65-69}$

Recent research indicates GSK-3 as a central regulator of stress-response and survival signaling that can promote both survival and death (depending on the cell type and experimental conditions) through its influence on crosstalk between key signaling pathways including the AKT, ERK1/2, JNK, and p38 pathways. ${ }^{30,31,33,70}$ The results obtained here show that GSK-3 inhibition led to decreased cell viability in a cancer cell line agreeing with previous studies that have found GSK-3 inhibition to be toxic in other cancer cell lines. ${ }^{71-73}$ In addition to identifying proteins likely to be involved in the response mechanism, the approach presented also revealed changes in protein roles that are suggestive of cell death progression. For example, significant 
plasma membrane degradation was only observed following $40 \mathrm{~min}$ exposure to TDZD- 8 at the 50 and $100 \mu \mathrm{M}$ doses. ERK1/2 radiality showed a decreasing trend as the dose of TDZD-8 increased. ERK1/2 radiality was the $2^{\text {nd }}$ highest of all proteins at the $10 \mu \mathrm{M}$ dose, and at the 40 , 50, and $100 \mu \mathrm{M}$ doses, ERK1/2 was the protein with the lowest radiality. Following $10 \mathrm{~h}$ exposure to TDZD-8, significant decreases in plasma membrane degradation were observed for the $30,40,50$, and $100 \mu \mathrm{M}$ doses. Only GSK-3 had a lower summation of radiality values than ERK1/2 across this dosing range. It is well known that ERK1/2 promotes survival under conditions of stress, ${ }^{74-77}$ and the dose-response curves constructed here suggest that a loss in ERK1/2 communication with the network may be a contributing step in the mechanism of toxicity for TDZD-8. JNK radiality tended to increase in response to increasing dose for both 40 min and $10 \mathrm{~h}$ exposure to TDZD-8, and JNK activation is a known death promoting mechanism carried out in cells to counter the effects of ERK1/2 survival signaling. ${ }^{77-79}$ Thus, the increase in JNK radiality and accompanied decrease in ERK1/2 radiality may play contributing roles in the mechanism of toxicity for TDZD-8.

It has been shown that GSK-3 can facilitate phosphorylation of pro-apoptotic proteins to promote apoptosis, or facilitate phosphorylation of anti-apoptotic proteins to neutralize their action as inhibitors of apoptosis. ${ }^{70}$ Following $40 \mathrm{~min}$ exposure, CREB had the $2^{\text {nd }}$ lowest radiality value out of all proteins at the $100 \mu \mathrm{M}$ dose of TDZD-8, and throughout the entire dosing range CREB radiality was one of the lowest of all proteins. It is well established that CREB activation is a survival promoting mechanism in cells exposed to stress. ${ }^{80,81}$ The relative phosphorylation data collected in this study show that CREB activation/phosphorylation is increased at all doses tested, and yet cellular viability is compromised. The radiality values for 
CREB do reflect a loss in communication between CREB and the signaling network as a whole; indicating that the network may not be responsive to CREB's survival promoting efforts.

Following exposure to TDZD-8 for 10 h, p53 (Ser15), JNK, p38, ERK1/2, and GSK-3 radiality values were altered the most out of all of the proteins. GSK-3 radiality showed a decreasing trend as the dose of TDZD-8 increased, reaching levels lower than any other protein, and displaying a different trend from all other proteins, making it clear that TDZD-8 had the greatest impact on GSK-3. This seems obvious knowing that TDZD-8 is a selective GSK-3 inhibitor, but had the identity of TDZD-8 not been known, the same conclusion could be drawn. The presented network analysis approach was able to identify specific proteins affected by the GSK-3 inhibitor following 40 min exposure, and was able to pinpoint GSK-3 itself as the main target following $10 \mathrm{~h}$ exposure. Simple inspection of the phosphorylation dataset alone cannot pinpoint these effects, and identification of protein targets would become even more difficult if the number of proteins monitored were to increase (which is necessary to be able to determine the mechanism of action for any unknown compound).

The approach presented here, as well as any study on signaling network responses, is limited by the number of proteins for which data is available. ${ }^{11}$ Here the phosphorylation or activation of 20 proteins was measured. In future studies, this data driven approach can easily be applied to significantly larger data sets containing 100's of proteins/nodes to provide a more comprehensive and integrated description of toxic MOAs for virtually any xenobiotic without reliance on any preexisting information. The presented approach is unique in that it describes toxic MOAs from a network perspective, and reflects the coordinated cellular response to exposure. It is advantageous in comparison to currently used approaches that utilize reference compounds and libraries, because it can be used to evaluate any compound of interest. 
Approaches relying on reference compounds are limited when it comes to characterizing MOAs that involve multiple signaling pathways, as reference compounds are commonly designed to be selective for specific pathways, and therefore, do not affect the network as broadly as other xenobiotics. The results obtained here reflect the specific response of cells to a specific set of experimental conditions. The utility of the presented approach in identifying the primary target of a xenobiotic and identifying proteins involved in the xenobiotics MOA has been demonstrated. This approach represents an attractive complimentary technique to those currently used to determine MOAs that is simple, comparatively low cost, high-throughput, and can be used to obtain toxicological information directly pertinent to human systems. 


\subsection{Bibliography}

(1) Scardoni, G., and Laudanna, C. (2012) Centralities Based Analysis of Complex Networks. New Frontiers in Graph Theory, 323-348. Dr. Yagang Zhang (Ed.), InTech, Available from: http://www.intechopen.com/books/new-frontiers-in-graph-theory/centralities-based-analysisof-networks

(2) Judson, R., Richard, A., Dix, D. J., Houck, K., Martin, M., Kavlock, R., Dellarco, V., Henry, T., Holderman, T., Sayre, P., Tan, S., Carpenter, T., and Smith, E. (2009) The toxicity data landscape for environmental chemicals. Environ Health Perspect, 117, 685-695.

(3) Erickson, B. (2013) Getting Real About Chemical Risks, In Chemical \& Engineering News 12-14.

(4) Fischetti, M. (2010) The Great Chemical Unknown, In Scientific American.

(5) National Research Council. (2010) Toxicity Pathway-Based Risk Assessment: Preparing for Paradigm Change: A Symposium Summary. Washington, DC: The National Academies Press.

(6) Carmichael, N., Bausen, M., Boobis, A. R., Cohen, S. M., Embry, M., Fruijtier-Polloth, C., Greim, H., Lewis, R., Bette Meek, M. E., Mellor, H., Vickers, C., and Doe, J. (2011) Using mode of action information to improve regulatory decision-making. Crit Rev Toxicol, 41, 175-186.

(7) Kholodenko, B. N. (2006) Cell signalling dynamics in time and space. Nat Rev Mol Cell Biol, 7, $165-176$.

(8) Newman, R. H., Hu, J., Rho, H. S., Xie, Z., Woodard, C., Neiswinger, J., Cooper, C., Shirley, M., Clark, H. M., Hu, S., Hwang, W., Jeong, J. S., Wu, G., Lin, J., Gao, X., Ni, Q., Goel, R., Xia, S., Ji, H., Dalby, K. N., Birnbaum, M. J., Cole, P. A., Knapp, S., Ryazanov, A. G., Zack, D. J., Blackshaw, S., Pawson, T., Gingras, A. C., Desiderio, S., Pandey, A., Turk, B. E., Zhang, J., Zhu, H., and Qian, J. (2013) Construction of human activity-based phosphorylation networks. Mol Syst Biol, 9, 655667. 
(9) Zhao, S., and lyengar, R. (2012) Systems pharmacology: network analysis to identify multiscale mechanisms of drug action. Annu Rev Pharmacol Toxicol, 52, 505-521.

(10) Csermely, P., Korcsmaros, T., Kiss, H. J., London, G., and Nussinov, R. (2013) Structure and dynamics of molecular networks: a novel paradigm of drug discovery: a comprehensive review. Pharmacol Ther, 138, 333-408.

(11) Mestres, J., Gregori-Puigjane, E., Valverde, S., and Sole, R. V. (2008) Data completeness--the Achilles heel of drug-target networks. Nat Biotechnol, 26, 983-984.

(12) Azzaoui, K., Hamon, J., Faller, B., Whitebread, S., Jacoby, E., Bender, A., Jenkins, J. L., and Urban, L. (2007) Modeling promiscuity based on in vitro safety pharmacology profiling data. Med Chem, $2,874-880$.

(13) Sonich-Mullin, C., Fielder, R., Wiltse, J., Baetcke, K., Dempsey, J., Fenner-Crisp, P., Grant, D., Hartley, M., Knaap, A., Kroese, D., Mangelsdorf, I., Meek, E., Rice, J. M., and Younes, M. (2001) IPCS conceptual framework for evaluating a mode of action for chemical carcinogenesis. Regul Toxicol Pharmacol, 34, 146-152.

(14) Boobis, A. R., Doe, J. E., Heinrich-Hirsch, B., Meek, M. E., Munn, S., Ruchirawat, M., Schlatter, J., Seed, J., and Vickers, C. (2008) IPCS framework for analyzing the relevance of a noncancer mode of action for humans. Crit Rev Toxicol, 38, 87-96.

(15) Meek, M. E. (2008) Recent developments in frameworks to consider human relevance of hypothesized modes of action for tumours in animals. Environ Mol Mutagen, 49, 110-116.

(16) Seed, J., Carney, E. W., Corley, R. A., Crofton, K. M., DeSesso, J. M., Foster, P. M., Kavlock, R., Kimmel, G., Klaunig, J., Meek, M. E., Preston, R. J., Slikker, W., Jr., Tabacova, S., Williams, G. M., Wiltse, J., Zoeller, R. T., Fenner-Crisp, P., and Patton, D. E. (2005) Overview: Using mode of action and life stage information to evaluate the human relevance of animal toxicity data. Crit Rev Toxicol, 35, 664-672. 
(17) Thompson, C. M., Haws, L. C., Harris, M. A., Gatto, N. M., and Proctor, D. M. (2011) Application of the U.S. EPA mode of action Framework for purposes of guiding future research: a case study involving the oral carcinogenicity of hexavalent chromium. Toxicol Sci, 119, 20-40.

(18) Berg, E. L., Yang, J., Melrose, J., Nguyen, D., Privat, S., Rosler, E., Kunkel, E. J., and Ekins, S. (2010) Chemical target and pathway toxicity mechanisms defined in primary human cell systems. $J$ Pharmacol Toxicol Methods, 61, 3-15.

(19) Houck, K. A., Dix, D. J., Judson, R. S., Kavlock, R. J., Yang, J., and Berg, E. L. (2009) Profiling bioactivity of the ToxCast chemical library using BioMAP primary human cell systems. J Biomol Screen, 14, 1054-1066.

(20) Nakatsu, N., Nakamura, T., Yamazaki, K., Sadahiro, S., Makuuchi, H., Kanno, J., and Yamori, T. (2007) Evaluation of action mechanisms of toxic chemicals using JFCR39, a panel of human cancer cell lines. Mol Pharmacol, 72, 1171-1180.

(21) U.S. EPA (2005) Guidelines for carcinogen risk assessment. Washington, DC EPA/630/P603/001F.

(22) Kunkel, E. J., Dea, M., Ebens, A., Hytopoulos, E., Melrose, J., Nguyen, D., Ota, K. S., Plavec, I., Wang, Y., Watson, S. R., Butcher, E. C., and Berg, E. L. (2004) An integrative biology approach for analysis of drug action in models of human vascular inflammation. Faseb J, 18, 1279-1281.

(23) Bender, A., Scheiber, J., Glick, M., Davies, J. W., Azzaoui, K., Hamon, J., Urban, L., Whitebread, S., and Jenkins, J. L. (2007) Analysis of pharmacology data and the prediction of adverse drug reactions and off-target effects from chemical structure. Med Chem, 2, 861-873.

(24) Hansch, C., and Selassie, C. (2007) Quantitative structure-activity relationship a historical perspective and the future. Comprehensive Medicinal Chemistry II. Vol. 4, Elsevier, Oxford.

(25) Paolini, G. V., Shapland, R. H., van Hoorn, W. P., Mason, J. S., and Hopkins, A. L. (2006) Global mapping of pharmacological space. Nat Biotechnol, 24, 805-815. 
(26) Fliri, A. F., Loging, W. T., Thadeio, P. F., and Volkmann, R. A. (2005) Biospectra analysis: model proteome characterizations for linking molecular structure and biological response. J Med Chem, $48,6918-6925$.

(27) Schultz, T. W., Cronin, M. T. D., Walker, J. D., and Aptula, A. O. (2003) Quantitative structureactivity relationships (QSARs) in toxicology: a historical perspective. J Mol Struct Theochem, 622, $1-22$.

(28) Kauvar, L. M., Villar, H. O., Sportsman, J. R., Higgins, D. L., and Schmidt, D. E., Jr. (1998) Protein affinity map of chemical space. J Chromatogr B Biomed Sci Appl, 715, 93-102.

(29) Bhalla, U. S., and lyengar, R. (1999) Emergent properties of networks of biological signaling pathways. Science, 283, 381-387.

(30) Jope, R. S., and Johnson, G. V. (2004) The glamour and gloom of glycogen synthase kinase-3. Trends Biochem Sci, 29, 95-102.

(31) Vene, R., and Tosetti, F. (2010) The role of glycogen synthase kinase-3 in the decision between cell survival and cell death. Emerging Signaling Pathways in Tumor Biology.

(32) Kaidanovich-Beilin, O., and Woodgett, J. R. (2011) GSK-3: Functional Insights from Cell Biology and Animal Models. Front Mol Neurosci, 4, 40-65.

(33) Jacobs, K. M., Bhave, S. R., Ferraro, D. J., Jaboin, J. J., Hallahan, D. E., and Thotala, D. (2012) GSK3beta: A Bifunctional Role in Cell Death Pathways. Int J Cell Biol, 2012, 930710.

(34) Verhees, K. J. P. (2013) No time to waste: a glycogen synthase kinase-3ß-glucocorticoid receptor signaling axis illuminated, In Graduate School VLAG p287, Wageningen University, the Netherlands.

(35) Cohen, P., and Goedert, M. (2004) GSK3 inhibitors: development and therapeutic potential. Nat Rev Drug Discov, 3, 479-487. 
(36) Collino, M., Thiemermann, C., Mastrocola, R., Gallicchio, M., Benetti, E., Miglio, G., Castiglia, S., Danni, O., Murch, O., Dianzani, C., Aragno, M., and Fantozzi, R. (2008) Treatment with the glycogen synthase kinase-3beta inhibitor, TDZD-8, affects transient cerebral ischemia/reperfusion injury in the rat hippocampus. Shock, 30, 299-307.

(37) Dajani, R., Fraser, E., Roe, S. M., Young, N., Good, V., Dale, T. C., and Pearl, L. H. (2001) Crystal structure of glycogen synthase kinase 3 beta: structural basis for phosphate-primed substrate specificity and autoinhibition. Cell, 105, 721-732.

(38) Doble, B. W., and Woodgett, J. R. (2003) GSK-3: tricks of the trade for a multi-tasking kinase. J. Cell Sci, 116, 1175-1186.

(39) Breen, E. C., Reynolds, S. M., Cox, C., Jacobson, L. P., Magpantay, L., Mulder, C. B., Dibben, O., Margolick, J. B., Bream, J. H., Sambrano, E., Martinez-Maza, O., Sinclair, E., Borrow, P., Landay, A. L., Rinaldo, C. R., and Norris, P. J. (2011) Multisite Comparison of High-Sensitivity Multiplex Cytokine Assays $\nabla \dagger$. Clin Vaccine Immunol, 18, 1229-1242.

(40) Medina, M., and Avila, J. (2010) Glycogen synthase kinase-3 (GSK-3) inhibitors for the treatment of Alzheimer's disease. Curr Pharm Des, 16, 2790-2798.

(41) Avila, J., and Hernandez, F. (2007) GSK-3 inhibitors for Alzheimer's disease. Expert Rev Neurothr, 7, 1527-1533.

(42) Klamer, G., Song, E., Ko, K. H., O'Brien, T. A., and Dolnikov, A. (2010) Using small molecule GSK3beta inhibitors to treat inflammation. Curr Med Chem, 17, 2873-2881.

(43) Phukan, S., Babu, V. S., Kannoji, A., Hariharan, R., and Balaji, V. N. (2010) GSK3beta: role in therapeutic landscape and development of modulators. Br J Pharmacol, 160, 1-19.

(44) Manning, G., Whyte, D. B., Martinez, R., Hunter, T., and Sudarsanam, S. (2002) The protein kinase complement of the human genome. Science, 298, 1912-1934. 
(45) Pavlopoulos, G., Secrier, M., Moschopoulos, C., Soldatos, T., Kossida, S., Aerts, J., Schneider, R., and Bagos, P. (2011) Using graph theory to analyze biological networks. BioData Min., 4, 10-37.

(46) Pržulj, N. (2007) Biological network comparison using graphlet degree distribution. Bioinformatics, 23, e177-e183.

(47) Pržulj, N., Corneil, D. G., and Jurisica, I. (2004) Modeling interactome: scale-free or geometric? Bioinformatics, 20, 3508-3515.

(48) Albert, R., and Barabasi, A.-L. (2002) Statistical mechanics of complex networks. Rev Mod Phys, 74, 47-97.

(49) Newman, M. E. J. (2003) The Structure and Function of Complex Networks. SIAM Rev, 45, 167256.

(50) Christensen, C., and Albert, R. (2007) Using Graph Concepts to Understand the Organization of Complex Systems. Int. J. Bifurcation Chaos, 17, 2201-2214.

(51) Bollobas, B. (1979) Graph Theory: An Introductory Course. Springer-Verlag, NY.

(52) Boccaletti, S., Latora, V., Moreno, Y., Chavez, M., and Hwang, D. U. (2006) Complex networks: Structure and dynamics. Phys Rep, 424, 175-308.

(53) Jeong, H., Tombor, B., Albert, R., Oltvai, Z. N., and Barabasi, A. L. (2000) The large-scale organization of metabolic networks. Nature, 407, 651-654.

(54) Grindrod, P., and Kibble, M. (2004) Review of uses of network and graph theory concepts within proteomics. Expert Rev Proteomics, 1, 229-238.

(55) Barabasi, A. L., and Oltvai, Z. N. (2004) Network biology: understanding the cell's functional organization. Nat Rev Genet., 5, 101-113.

(56) Albert, R. (2005) Scale-free networks in cell biology. J Cell Sci, 118, 4947-4957.

(57) Huber, W., Carey, V. J., Long, L., Falcon, S., and Gentleman, R. (2007) Graphs in molecular biology. BMC Bioinf, 8, s8. 
(58) Koschutzki, D., and Schreiber, F. (2008) Centrality Analysis Methods for Biological Networks and Their Application to Gene Regulatory Networks. Gene Regul Syst Biol, 2, 193-201.

(59) Ma'ayan, A. (2009) Insights into the Organization of Biochemical Regulatory Networks Using Graph Theory Analyses. J Biol Chem, 284, 5451-5455.

(60) Brandes, U. (2001) A faster algorithm for betweenness centrality. J Math Soc, 25, 163-177.

(61) Valente, T., W, and Foreman, R., K. (1998) Integration and radiality: Measuring the extent of an individual's connectedness and reachability in a network. Social Networks, 20, 89-105.

(62) Borgatti, S. P., and Everett, M. G. (2006) A Graph-theoretic perspective on centrality. Social Networks, 28, 466-484.

(63) Scardoni, G., Petterlini, M., and Laudanna, C. (2009) Analyzing biological network parameters with CentiScaPe. Bioinformatics, 25, 2857-2859.

(64) Boyd, J., Vrana, J. A., and Williams, H. N. (2012) In vitro approach to predict post-translational phosphorylation response to mixtures. Toxicology, 313, 113-121.

(65) Kim, S. D., Yang, S.-I. Y., Kim, H.-C., Shin, C. Y., and Ho Ko, K. (2007) Inhibition of GSK-3 $\beta$ mediates expression of MMP-9 through ERK1/2 activation and translocation of NF-KB in rat primary astrocyte. $1186,12-20$.

(66) Ngok-Ngam, P., Watcharasit, P., Thiantanawat, A., and Satayavivad, J. (2013) Pharmacological inhibition of GSK3 attenuates DNA damage-induced apoptosis via reduction of p53 mitochondrial translocation and Bax oligomerization in neuroblastoma SH-SY5Y cells. Cell Mol Biol Lett, 18, 58-74.

(67) Gotschel, F., Kern, C., Lang, S., Sparna, T., Markmann, C., Schwager, J., McNelly, S., von Weizsacker, F., Laufer, S., Hecht, A., and Merfort, I. (2008) Inhibition of GSK3 differentially modulates NF-kappaB, CREB, AP-1 and beta-catenin signaling in hepatocytes, but fails to promote TNF-alpha-induced apoptosis. Exp Cell Res, 314, 1351-1366. 
(68) Wang, Q., Zhou, Y., Wang, X., and Evers, B. M. (2006) Glycogen synthase kinase-3 is a negative regulator of extracellular signal-regulated kinase. Oncogene, 25, 43-50.

(69) Takada, Y., Fang, X., Jamaluddin, M. S., Boyd, D. D., and Aggarwal, B. B. (2004) Genetic deletion of glycogen synthase kinase-3beta abrogates activation of IkappaBalpha kinase, JNK, Akt, and p44/p42 MAPK but potentiates apoptosis induced by tumor necrosis factor. J Biol Chem, 279, 39541-39554.

(70) Beurel, E., and Jope, R. S. (2006) The paradoxical pro- and anti-apoptotic actions of GSK3 in the intrinsic and extrinsic apoptosis signaling pathways. Prog Neurobiol, 79, 173-189.

(71) Dickey, A., Schleicher, S., Leahy, K., Hu, R., Hallahan, D., and Thotala, D. K. (2011) GSK-3beta inhibition promotes cell death, apoptosis, and in vivo tumor growth delay in neuroblastoma Neuro-2A cell line. J Neurooncol, 104, 145-153.

(72) Korur, S., Huber, R. M., Sivasankaran, B., Petrich, M., Morin, P., Jr., Hemmings, B. A., Merlo, A., and Lino, M. M. (2009) GSK3beta regulates differentiation and growth arrest in glioblastoma. PLoS One, 4, e7443.

(73) Kotliarova, S., Pastorino, S., Kovell, L. C., Kotliarov, Y., Song, H., Zhang, W., Bailey, R., Maric, D., Zenklusen, J. C., Lee, J., and Fine, H. A. (2008) Glycogen synthase kinase-3 inhibition induces glioma cell death through c-MYC, nuclear factor-kappaB, and glucose regulation. Cancer Res, 68, 6643-6651.

(74) Blank, J. L., Gerwins, P., Elliott, E. M., Sather, S., and Johnson, G. L. (1996) Molecular cloning of mitogen-activated protein/ERK kinase kinases (MEKK) 2 and 3. Regulation of sequential phosphorylation pathways involving mitogen-activated protein kinase and c-Jun kinase. J Biol Chem, 271, 5361-5368.

(75) Karandikar, M., Xu, S., and Cobb, M. H. (2000) MEKK1 binds raf-1 and the ERK2 cascade components. J Biol Chem, 275, 40120-40127. 
(76) Waetzig, V., and Herdegen, T. (2005) MEKK1 controls neurite regrowth after experimental injury by balancing ERK1/2 and JNK2 signaling. Mol Cell Neurosci, 30, 67-78.

(77) Cargnello, M., and Roux, P. P. (2011) Activation and Function of the MAPKs and Their Substrates, the MAPK-Activated Protein Kinases. Microbiol Mol Biol Rev, 75, 50-83.

(78) Xia, Z., Dickens, M., Raingeaud, J., Davis, R. J., and Greenberg, M. E. (1995) Opposing effects of ERK and JNK-p38 MAP kinases on apoptosis. Science, 270, 1326-1331.

(79) Junttila, M. R., Li, S.-P., and Westermarck, J. (2008) Phosphatase-mediated crosstalk between MAPK signaling pathways in the regulation of cell survival. FASEB J, 22, 954-965.

(80) Ballif, B. A., and Blenis, J. (2001) Molecular mechanisms mediating mammalian mitogenactivated protein kinase (MAPK) kinase (MEK)-MAPK cell survival signals. Cell Growth Differ, 12, 397-408.

(81) Mayr, B., and Montminy, M. (2001) Transcriptional regulation by the phosphorylationdependent factor CREB. Nat Rev Mol Cell Biol, 2, 599-609. 


\section{Chapter 3}

Characterization of Network Perturbations in Response to Inhibitor Induced Mitochondrial Stress 


\subsection{Introduction}

Historically, toxicological assessments have been based on common endpoint evaluations such as death, cancer, or an increase or decrease in levels of molecules associated with particular biological processes. Modern toxicological evaluations have evolved to consider toxicity as a perturbation of biological pathways or networks. ${ }^{1-4}$ As such, toxicity testing approaches are shifting from common endpoint evaluations to pathway based approaches, where the degree of

perturbation of select biological pathways is monitored. ${ }^{3,4}$ Pathway based approaches represent a significant improvement to current toxicological assessments, but methods of analysis to determine the inter-relationships between potentially affected pathways are needed to fully understand the consequences of toxic exposure. This chapter demonstrates the utility of graph theoretical analyses in assessing the toxicological effects of chemical exposure from a network perspective. The network approach presented in chapter 2 is used to characterize the effects of the mitochondrial complex I inhibitor, deguelin, on HepG2 cells on a dose-to-dose basis. The approach depicts low dose perturbations in the balance between MAPK signaling pathways, providing an informative and sensitive means of assessing toxicological effects on biological systems.

\subsubsection{Mitogen Activated Protein Kinase Crosstalk and Cell Death}

The signaling proteins that have been most closely associated with chemical exposure stress are the mitogen-activated protein kinases (MAPKs). ${ }^{3,5-9}$ There are seven known separate families of mammalian MAPKs, and the most extensively studied of these groups are the ERK1/2, JNK, and p38 kinase pathways. ${ }^{10}$ ERK1/2 pathway activation is generally associated with cell growth, proliferation, and survival, while p38 and JNK signaling pathways are typically associated with stress responses and apoptosis. ${ }^{10}$ These three MAPK families have generally 
been thought to be involved in separate linear signaling cascades, since each MAPK is activated by different upstream kinases, which are directed by distinct extracellular stimuli. ${ }^{9,11}$ However, it has been shown that crosstalk between these MAPK pathways does exist, and that the crosstalk between these pathways may be crucial to their ability to coordinate certain cellular stress responses including survival and inflammation. ${ }^{11-13}$ A network depicting the modular organization of MAPK signaling is shown in Figure 3-1. It has been suggested that under normal physiological conditions MAPK signaling pathways are linear with no crosstalk, but that pathological conditions and stresses, such as exposure to xenobiotics, may drive crosstalk between these pathways. ${ }^{14-17}$ The balance between ERK1/2, p38, and JNK signaling pathways can push cells towards life or death. ${ }^{9,15,18}$ The ability to appropriately capture exposure-induced alterations in the balance between distinct MAPK signaling pathways provides a unique view of pathway based toxicity testing that can yield valuable insight into the underlying biological response to chemical insults.
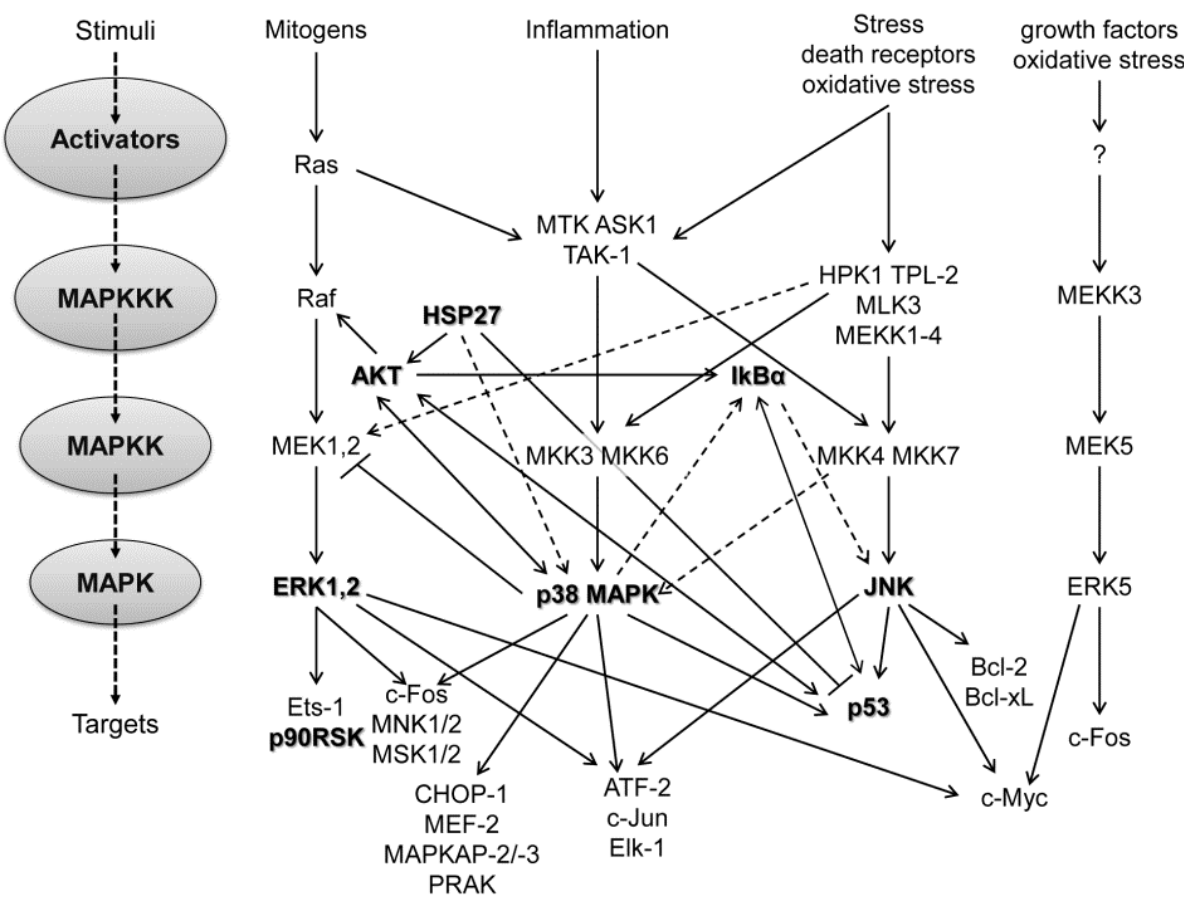

Figure 3-1. Modular organization of MAPK signaling pathways. 


\subsubsection{Graph Theoretical Identification of Low-dose Network Perturbations}

While advances in high-throughput data collection techniques have led to a rapid increase in the application of graph theory analyses to the study of biological signaling networks, it has yet find application in the field of toxicology, and specifically has never been applied to dose. The determination of concentration ranges that lead to adverse health effects over time is extremely important to toxicological assessments. ${ }^{19}$ This requires approaches that are capable of assessing the effects that chemical exposures have on entire networks on a dose-to-dose basis. Ideally, toxic effects should be identified before gross toxicological effects (with irreversible consequences) are manifested. To do this, subtle alterations in normal cellular functioning caused by low-dose exposures must be identified. Changes in the networked relationships between signaling proteins (specifically MAPKs that influence cell life and death) are likely to precede more direct toxic manifestations and represent an attractive early descriptor of the toxic effects of exposure. The graph theoretical approach presented in this work can be used to describe these networked relationships following exposure to low doses of toxic compounds.

In this chapter, HepG2 cells were exposed to increasing doses of the mitochondrial inhibitor deguelin. ${ }^{20}$ Graph theory was used to describe the networked protein response at each dose based on the levels of phosphorylation of 8 proteins (ERK1/2, JNK, p38, AKT, HSP27, I $\mathrm{B} \alpha, \mathrm{p} 53$, and $\mathrm{p} 90 \mathrm{RSK})$ related to mitochondrial stress. Two secondary inhibitors, SB202190 and SB202474 were dosed in combination with deguelin to further probe the network response. The work presented in this chapter has been published in the American Chemical Society journal Chemical Research in Toxicology. ${ }^{21}$ 


\subsubsection{Deguelin, SB202190, and SB202474}

Deguelin was chosen as the inhibitor of interest in this study due to its ability to induce mitochondrial stress, which is an important focus of many toxicity assays. Deguelin inhibits Nicotinamide Adenine Dinucleotide (NADH): oxidoreductase (complex I of the electron transport chain), AKT, and HSP90, and exhibits anticancer activity. ${ }^{22-26}$ Mitochondria have a significant impact on cellular physiology due to their roles in ATP production, reactive oxygen species (ROS) generation, $\mathrm{Ca}^{2+}$ homeostasis, and cell death. ${ }^{27,} 28$ Alterations in the signaling networks involved in the regulation of these mitochondrial functions have been implicated as contributing factors to many diseases including cancer. ${ }^{28-30}$ Further, mitochondria integrate a multitude of signaling events, contribute to cellular death via mitochondrial membrane permeability, and are common targets of both therapeutic and environmental xenobiotics. ${ }^{31-36}$

SB202190 and SB202474 were selected as secondary inhibitors (each dosed in combination with deguelin) to further investigate the balance between the MAPK signaling pathways following chemically induced stress. SB202190 inhibits both JNK and p38, while SB20474 inhibits JNK but not p38. Both compounds exert the same effects on all of the other proteins studied, and were chosen to allow comparison of just partial JNK inhibition to partial JNK and p38 inhibition during deguelin exposure. The structures of deguelin, SB202190, and SB202474 are shown in Figure 3-2. 

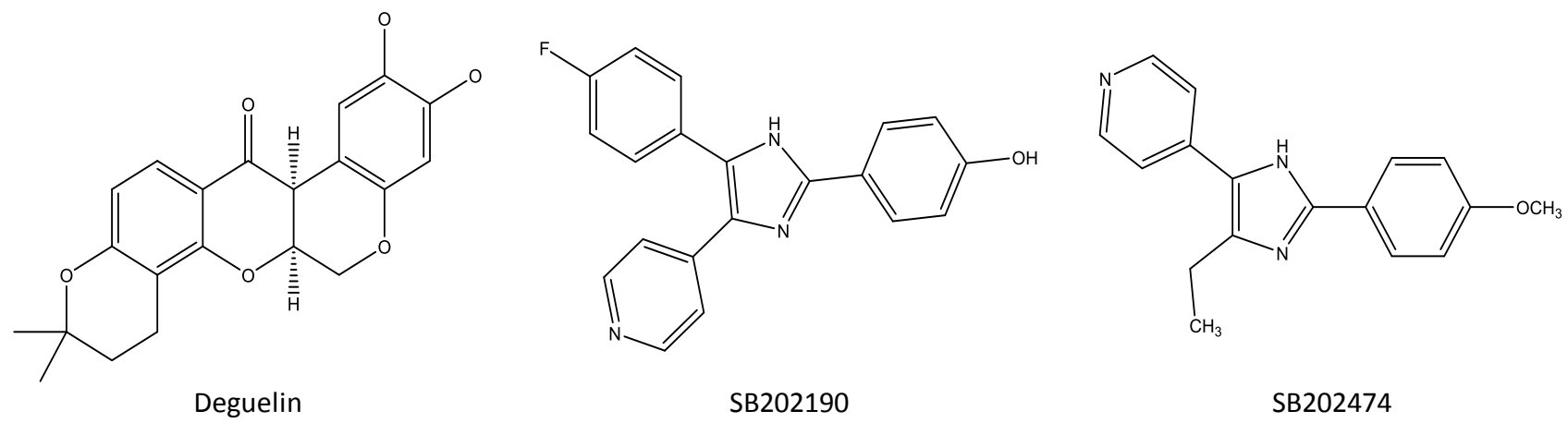

Figure 3-2. Deguelin, SB202190, and SB202474

\subsection{Experimental Procedures}

\subsubsection{Materials}

Deguelin was obtained from Sigma Aldrich (St. Louis, MO). SB202190, and SB202474 were obtained from Calbiochem (San Diego, California). RPMI-1640, sodium pyruvate, HEPES, L-glutamine, fetal bovine serum, and penicillin-streptomycin were obtained from Invitrogen (Carlsbad, CA). HepG2 cells were obtained from American Type Culture Collection (Manassas, VA). Bio-plex beads, lysis buffer, antibodies, and reagents necessary for determination of relative phosphorylation were obtained from Bio-Rad (Hercules, CA).

\subsubsection{Cell culture.}

HepG2 cells were cultured in RPMI-1640, supplemented with $1 \mathrm{mM}$ sodium pyruvate, 5 mM HEPES, 2 mM L-Glutamine, 10\% fetal bovine serum (FBS), $100 \mathrm{U} / \mathrm{ml}$ penicillin, and 100 $\mu \mathrm{g} / \mathrm{ml}$ streptomycin. Cells were maintained in a humidified atmosphere at $37^{\circ} \mathrm{C}, 5 \% \mathrm{CO}_{2}$ and passaged at $80 \%$ confluence.

\subsubsection{Dosing.}

Cells were seeded into clear-bottom, black-sided 96-well plates at a density of $4 \times 10^{4}$ cells per well and allowed to grow for $24 \mathrm{~h}$. Media was then aspirated from wells, and cells were challenged with varying concentrations of single and mixed compounds in fresh media. 
Deguelin was prepared in dimethyl sulfoxide (DMSO) to concentrations of $0.001 \mu \mathrm{M}$ to $100 \mu \mathrm{M}$. SB202190 and SB202474 were prepared in DMSO to their manufacturer specified $\mathrm{IC}_{50}$ concentrations of $350 \mathrm{nM}$ and $34 \mathrm{nM}$, respectively.

\subsubsection{MTT Viability Assay.}

After 400 min of exposure to single compounds or mixtures of compounds, cell viability was determined using the 3-(4,5-dimethylthiazolyl-2)-2,5-diphenyltetrazolium bromide (MTT) assay. This 400 min time point was chosen based on a previous study showing that changes in cellular oxygen consumption point to critical signaling events occurring at $400 \mathrm{~min}$ of exposure to deguelin. ${ }^{37}$ The MTT assay was performed according to the manufacturer's protocol. The assay is based on the reduction of tetrazolium MTT to colored formazan by metabolically active

cells, in part by the action of dehydrogenase enzymes, to generate reducing equivalents such as NADH and Nicotinamide Adenine Dinucleotide Phosphate (NADPH). MTT reagent was added to the wells of the microplate, and after two hours of incubation at $37^{\circ} \mathrm{C}$, intracellular formazan crystals were solubilized with the provided detergent solution. Absorbance values were obtained using the Safire2 microplate reader (Tecan US, Raleigh, NC) with a measurement wavelength of $570 \mathrm{~nm}$ and a reference wavelength of $700 \mathrm{~nm}$, read from the bottom. From this, relative viability was determined by subtracting the absorbance of blank wells (consisting of cell culture media, the highest dose of the inhibitor being tested, all MTT reagents, but no cells) from all treatments and controls. The ratio of the blank-corrected absorbance values for wells containing treated cells relative to the wells containing controls (consisting of everything in the treated wells, except the inhibitor) was determined. 


\subsubsection{Bio-plex Multiplex Immunoassay}

HepG2 cells were plated at 500,000 cells per well in 12-well tissue culture plates in Dulbecco's Modified Eagle Medium (DMEM) growth media (10\% heat-inactivated FBS) supplemented with $0.1 \%$ fatty acid-free bovine serum albumin (BSA) (Sigma, St. Louis, MO) for $24 \mathrm{~h}$. Cells were then treated with increasing doses of deguelin $(0.001,0.01,0.1,1.0,10,100$ $\mu \mathrm{M})$ in $1 \%$ DMSO alone, or in binary combination with the $\mathrm{IC}_{50}$ concentrations of $350 \mathrm{nM}$ SB202190 or 34nM SB20474. After an incubation period of $400 \mathrm{~min}$, cells were lysed with lysis buffer (Bio-Rad, Hercules, CA) supplemented with $500 \mu \mathrm{M}$ phenylmethanesulfonylfluoride (PMSF) (Sigma, St. Louis, MO) and phosphatase inhibitors (Bio-Rad, Hercules, CA). Total protein concentration was determined using the DC Protein Assay (Bio-Rad, Hercules, CA) according to the manufacturer's instructions. Beads containing antibodies against phosphorylated ERK1/2 (Thr202/Tyr204, Thr185/Tyr187), AKT (Ser473), HSP27 (Ser78), IкB $\alpha$ (Ser32/Ser36), JNK (Thr183/Tyr185), p38MAPK (Thr180/Tyr182), p53 (Ser15), and p90RSK (Thr359/Ser363) were obtained from Bio-Rad (Hercules, CA). Relative protein phosphorylation was determined using the multiplexed bead-based assays and the Bio-plex suspension array system according to the manufacturer's instructions (Bio-Rad, Hercules, CA). Relative fluorescence values were calculated from the raw fluorescence levels after subtracting background (no cells) and normalizing to control cells receiving only 1\% DMSO. All experiments were performed in duplicate and error bars reflect the standard error of the mean (SEM). 


\subsubsection{Data Analysis.}

Two-way ANOVA's with Bonferroni's post tests were performed using GraphPad Prism version 5.03 for Windows, GraphPad Software (San Diego, California). Euclidean distances $(E)$ were determined with SAS JMP (Carey, NC) using the equation

$$
E(v, \omega)=\sqrt{\sum_{i=1}^{n}\left(\left(\text { Relative Phosphorylation } v_{i}\right)-\left(\text { Relative Phosphorylation } \omega_{i}\right)\right)^{2}}
$$

Where $v$ and $\omega$ represent the 2 protein nodes for which the distance between is being calculated, and $n$ represents the number of replicates for which phosphorylation data was collected. All Euclidean distances were multiplied by 100 to facilitate network parameter calculations in Cytoscape. There was no set threshold value for the existence of an edge, and thus all distance values were used in the calculation of radiality. The node centrality parameter, radiality $\left(C_{\text {rad }}(v)\right)$, is calculated using the equation

$$
C_{\text {rad }}(v)=\frac{\sum_{\omega \in N}\left(\Delta_{G}+1-\operatorname{dist}(v, \omega)\right)}{n-1}
$$

where $\Delta_{\mathrm{G}}$ is the network diameter (longest path length connecting any 2 nodes in the network), $n$ is the number of nodes in the network, and $\operatorname{dist}(v, \omega)$ is the shortest possible summation of Euclidean distance weighted edge lengths connecting node $v$ to node $\omega . \Delta_{\mathrm{G}}$ represents the compactness of the network, and can be interpreted as a relative estimate of protein communication. To interpret centrality from $C_{\text {rad }}(v)$ : if the radiality of a node, $v$, is high, then 
the node is generally close to all other nodes in the network, and therefore is considered central. ${ }^{38}$ Centrality parameters can be interpreted in various ways, and the interpretation often depends on the specific application of the analysis. ${ }^{39}$ Radiality as calculated here, concisely and simultaneously describes each protein's phosphorylation mediated relation to all other proteins. Mechanistically, this means that a protein/node with low radiality is not highly integrated within the network, and is not highly central to network communication. Therefore the other proteins are more central to this networks response for a given exposure condition. Radiality parameters were normalized to the average radiality values for all proteins in the network, making proteins with a radiality higher than 1 , more central than average, and proteins with radiality lower than 1 , less central than average.

\subsection{Results}

\subsubsection{Phosphorylation Response and Viability.}

The relative phosphorylation levels (normalized to control cells that received less than 1\% DMSO) for ERK1/2, JNK, p38, AKT, HSP27, IкB $\alpha, \mathrm{p} 53$, and p90RSK were measured following $400 \mathrm{~min}$ exposure to increasing doses $(0.001,0.01,0.1,1,10$, and $100 \mu \mathrm{M})$ of deguelin alone (Figure 3-3A). The most exaggerated response to treatment with deguelin alone was observed for $\mathrm{p} 38$ at the $10 \mu \mathrm{M}$ dose. At the $10 \mu \mathrm{M}$ dose of deguelin, p38 levels were $4.9 \pm$ 0.1 times greater than controls. SB202190, a combination p38 and JNK inhibitor, was examined and the relative phosphorylation levels for the 8 proteins were determined in cells treated with its single $\mathrm{IC}_{50}$ concentration of $350 \mathrm{nM}$ (Figure 3-3B). SB202190 was chosen due to the availability of a negative control, SB202474. SB202474 is shown to exert the same effects as SB202190 on cellular signaling, except for inhibition of p38..$^{40-42}$ The $350 \mathrm{nM}$ dose of SB202190 reduced p38 
phosphorylation to $0.61 \pm 0.02$ times that of control cells. Cells were exposed to the $\mathrm{IC}_{50}$ dose of SB202474 (34 nM), and relative p38 phosphorylation levels were $1.2 \pm 0.1$ times that of control cells (Figure 3-3B). Two-way ANOVA comparing the 8 proteins' relative phosphorylation responses to the $\mathrm{IC}_{50}$ doses of SB202190 and SB202474 shows that the only statistical difference at $p<0.01$ was for $\mathrm{p} 38$. The $\mathrm{IC}_{50}$ doses of SB202190 and SB202474 decreased relative JNK phosphorylation to $0.416 \pm 0.004$ and $0.519 \pm 0.004$, respectively. Relative IkB $\alpha$ phosphorylation decreased to $0.33 \pm 0.03$ following exposure to the $\mathrm{IC}_{50}$ dose of SB202190 and to $0.329 \pm 0.001$ following exposure to $\mathrm{SB} 202474$ at its $\mathrm{IC}_{50}$ concentration. 
A
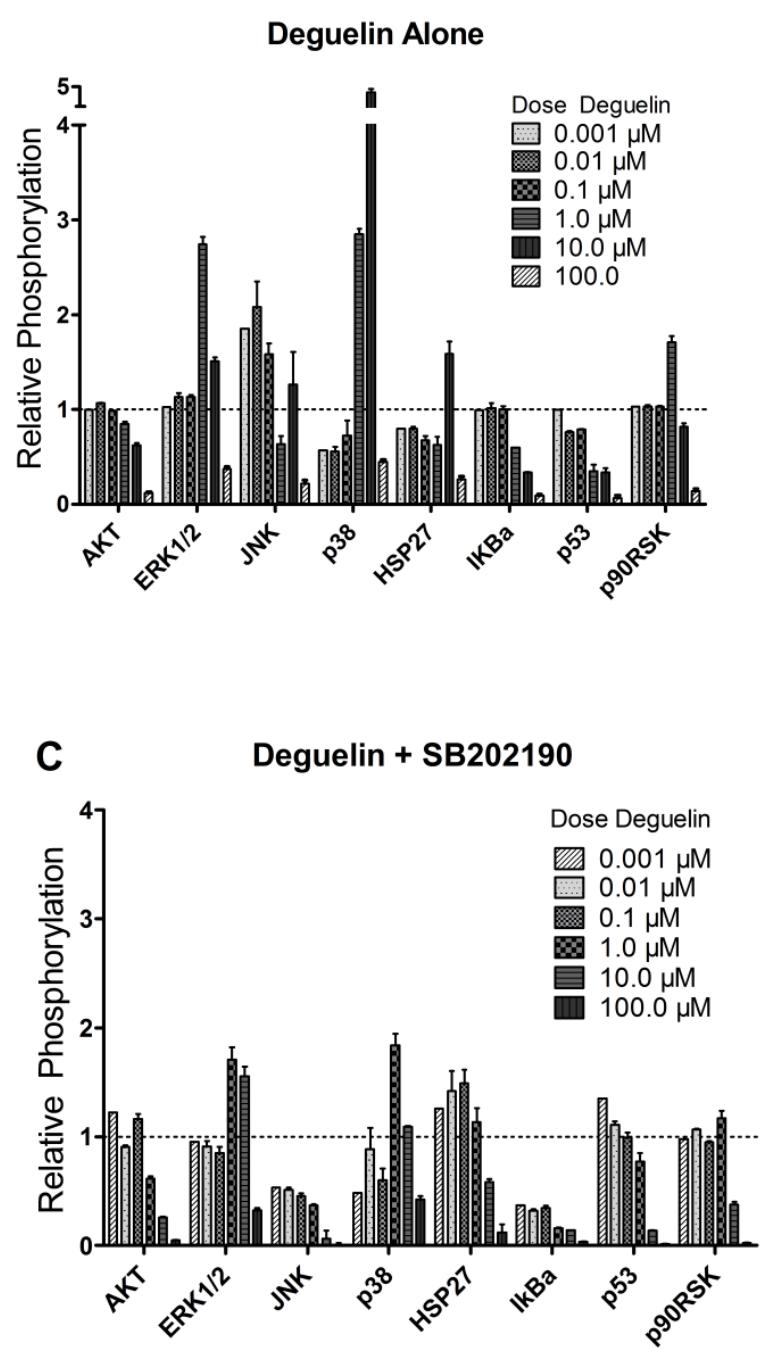

B

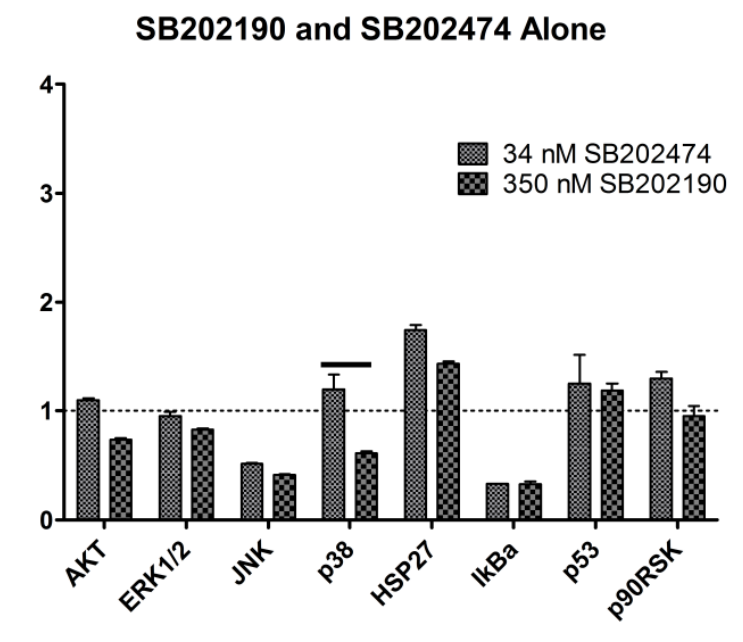

D Deguelin + SB202474

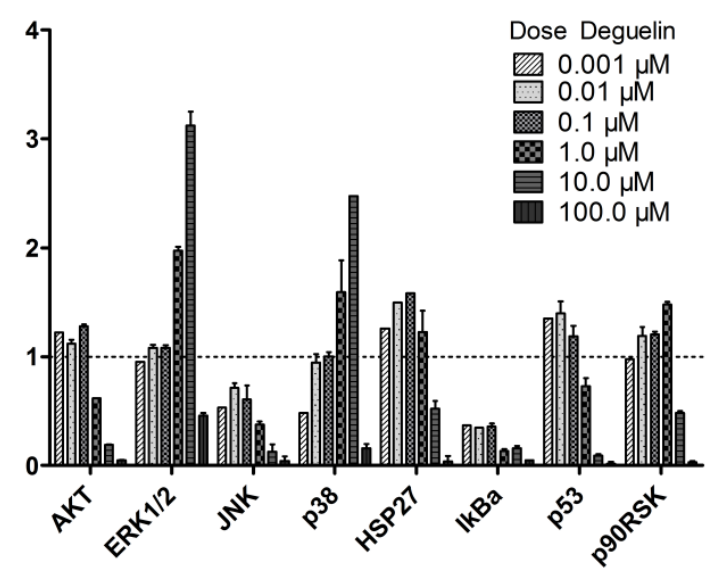

Figure 3-3. Phosphorylation response to inhibitor treatments. HepG2 cells were exposed to inhibitor treatments for $400 \mathrm{~min}$. Shown are the phosphorylation levels relative to the vehicle control (1\% DMSO) as determined by multi-plexed bead immunoassay. Observed data points represent the average of two replicates. Error bars represent the standard error of the mean. Bars marked with $(-)$ on figure B represent significantly different $(p<0.01)$ responses when comparing SB202190 at its IC50 concentration to SB202474 at its IC50 concentration.

To probe the network response to deguelin, cells were exposed to increasing doses of deguelin in combination with the $350 \mathrm{nM}$ dose of SB202190, and relative protein phosphorylation was determined (Figure 3-3C). The increased p38 phosphorylation observed in response to deguelin alone was inhibited by SB202190 (Figure 3-3C) with the level of p38 
phosphorylation at the $10 \mu \mathrm{M}$ dose of deguelin only reaching $1.1 \pm 0.01$ times that of control treated cells. Cells were then exposed to increasing doses of deguelin in combination with 34 nM SB202474, and relative protein phosphorylation was measured (Figure 3-3D). In cells treated with $10 \mu \mathrm{M}$ deguelin in combination with $34 \mathrm{nM}$ SB202474, the measured level of phosphorylated p38 was $2.5 \pm 0.1$ times greater than control cells. Statistically significant differences in protein phosphorylation responses of cells treated with each inhibitor combination are listed in Table 3-1.

Table 3-1. Significant Differences in Phosphorylation Response ${ }^{a}$

\begin{tabular}{|c|c|c|c|c|c|c|}
\hline \multirow[b]{2}{*}{$\begin{array}{l}\text { Deguelin vs Deguelin + } 350 \mathrm{nM} \\
\text { SB202190 }\end{array}$} & \multicolumn{6}{|c|}{ Dose Deguelin $(\mu \mathrm{M})$} \\
\hline & 0.001 & 0.01 & 0.1 & 1 & 10 & 100 \\
\hline AKT & $\star \star \star \star$ & $\star \star \star \star *$ & $\star \star \star \star$ & $\star \star \star \star$ & $\star \star \star \star$ & \\
\hline ERK1/2 & ** & ** & & $* * *$ & $* *$ & \\
\hline JNK & $\star \star \star *$ & $\star \star \star *$ & ** & & 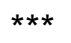 & \\
\hline p38 & & & & $\star * *$ & $\star \star \star *$ & \\
\hline HSP27 & & ** & & & $* \star *$ & \\
\hline $\begin{array}{l}\text { IkBa } \\
\text { p53 }\end{array}$ & $* \star \star$ & $\star * *$ & *** & $* * *$ & ** & \\
\hline p90RSK & & & & $\star \star \star$ & & \\
\hline \multicolumn{7}{|l|}{$\begin{array}{l}\text { Deguelin vs Deguelin + } 34 \mathrm{nM} \\
\text { SB202474 }\end{array}$} \\
\hline AKT & $\star \star \star \star$ & $\star \star \star \star$ & 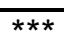 & 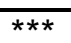 & $\star \star \star \star$ & \\
\hline ERK $1 / 2$ & $\star *$ & & & $\star \star \star *$ & *** & \\
\hline JNK & ** & & & & $* * *$ & \\
\hline p38 & & & & $\star \star \star *$ & 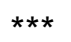 & \\
\hline HSP27 & $* * *$ & $* \star *$ & & & $* \star *$ & \\
\hline lkBa & $\star \star \star \star$ & $* * *$ & $\star \star \star *$ & $* * *$ & & \\
\hline $\begin{array}{l}\text { p53 } \\
\text { p90RSK }\end{array}$ & & & $\star \star * *$ & $\star * *$ & & \\
\hline \multicolumn{7}{|l|}{$\begin{array}{l}\text { Deguelin + } 350 \mathrm{nM} \text { SB202190 vs } \\
\text { Deguelin + } 34 \mathrm{nM} \text { SB202474 }\end{array}$} \\
\hline AKT & & $\star \star \star \star$ & ** & & & \\
\hline ERK $1 / 2$ & & & & & $\star \star \star *$ & \\
\hline JNK & & & & & & \\
\hline p38 & & & & & $* * *$ & \\
\hline HSP27 & & & & & & \\
\hline IkBa & & & & & & \\
\hline p53 & & & & & & \\
\hline p90RSK & ** & & & ** & $* * *$ & \\
\hline
\end{tabular}

(a) Statistically significant differences in relative protein phosphorylation when comparing different inhibitor treatments. (***) indicates $p<0.001,(* *)$ indicates $p<0.01$, and $(*)$ indicates $p<0.05$. 
Figure 3-4 depicts relative viability (normalized to control cells that received less than $1 \%$ DMSO) for cells treated with the inhibitors for $400 \mathrm{~min}$. The only treatments that led to statistically significant $(p<0.01)$ changes in relative viability in comparison to controls were for cells treated with $34 \mathrm{nM}$ SB202474 in combination with 10 or $100 \mu \mathrm{M}$ doses of deguelin. HepG2 relative viability was reduced to $62 \pm 3 \%$ when treated with $10 \mu \mathrm{M}$ deguelin in combination with $34 \mathrm{nM}$ SB202474 and $34 \pm 3 \%$ when treated with $100 \mu \mathrm{M}$ deguelin in combination with 34 nM SB202474.
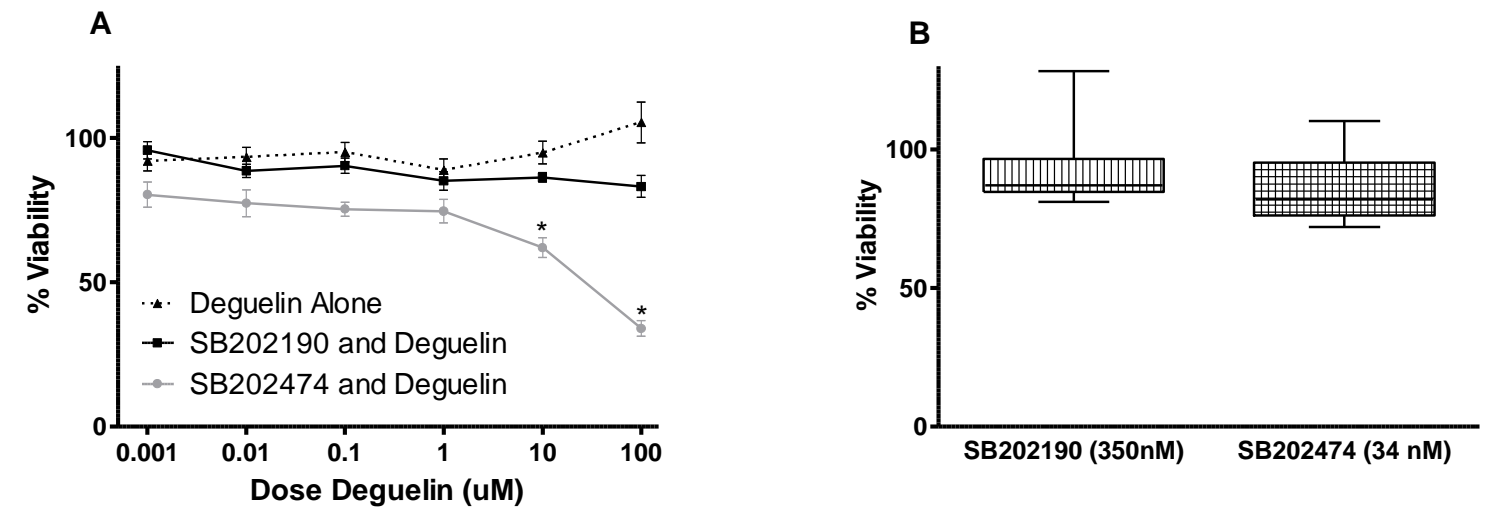

Figure 3-4. Relative viability at 400 min as determined by MTT assay. (A) HepG2 cells were treated with increasing doses of deguelin alone, increasing doses of deguelin in combination with a constant $350 \mathrm{nM}$ dose of SB202190, or increasing doses of deguelin in combination with a constant 34 nM dose SB202474. Data are presented in terms of percent viability of the vehicle control (1\% DMSO). Observed data points represent the average of three independent experiments. Error bars represent the standard error of the mean. Statistically significant $(p<0.01)$ changes in viability in comparison to control treated cells are marked with $\left(^{*}\right)$. (B) Box-and-whisker plots for cells treated with SB202190 at its single IC ${ }_{50}$ dose, $350 \mathrm{nM}$ or SB202474 at its $\mathrm{IC}_{50}$ value, $34 \mathrm{nM}$. Whisker ends represent the minimum and maximum values. 


\subsubsection{Euclidean Distances and Radiality}

\subsubsection{Deguelin Alone.}

To obtain network edge weight values for graph theory analysis, Euclidean distances between each pair of proteins were calculated based on their relative phosphorylation responses (normalized to control cells that received less than $1 \%$ DMSO) to each individual dose of deguelin. Table 3-2 shows the Euclidean distance values (in order of shortest distance to longest), calculated based on the phosphorylation levels of proteins treated with increasing doses of deguelin alone. A low distance value means that the protein responses to the specific conditions are similar, providing evidence of a connection or relationship between the two proteins. Conversely, a high distance value means that the protein responses are not similar, and that there is little evidence of a relationship between the two proteins. The distances between ERK1/2, p38, and JNK were all relatively high (all distances being greater than the average distance value) at the $0.001,0.01$, and $0.1 \mu \mathrm{M}$ doses of deguelin. At the $1.0 \mu \mathrm{M}$ dose of deguelin, the distance between p38 and ERK1/2 decreased to less than the average protein distance for all proteins, while the p38-JNK and JNK-ERK1/2 distances were well above average. At the $10 \mu \mathrm{M}$ dose of deguelin the distance between p38 and ERK1/2 increased and was much greater than average, while the distance between JNK and ERK1/2 decreased to below average. The p38-JNK distance remained high at the $10 \mu \mathrm{M}$ dose. At the $100 \mu \mathrm{M}$ dose of deguelin, the distance between p38 and ERK1/2 decreased to less than average; the JNK-ERK1/2 distance was near average; and the p38-JNK distance was above average. Three graphs depicting the characteristics of the different network responses to the 3 inhibitor treatments at the $10 \mu \mathrm{M}$ dose of deguelin are shown in Figure 3-5. 


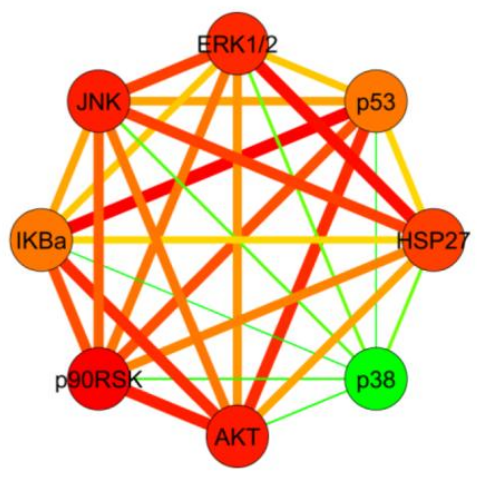

$10 \mu \mathrm{M}$ Deguelin

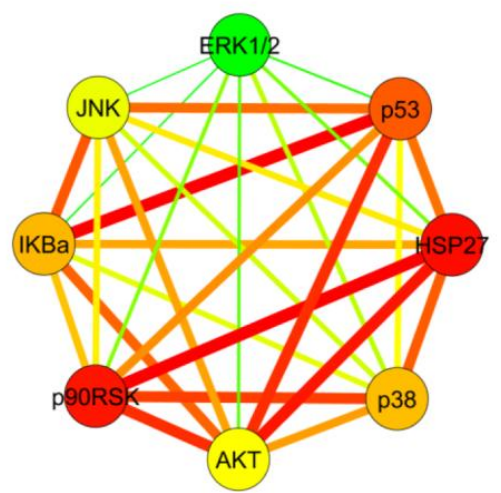

$10 \mu \mathrm{M}$ Deguelin $+350 \mathrm{nM}$ SB202190

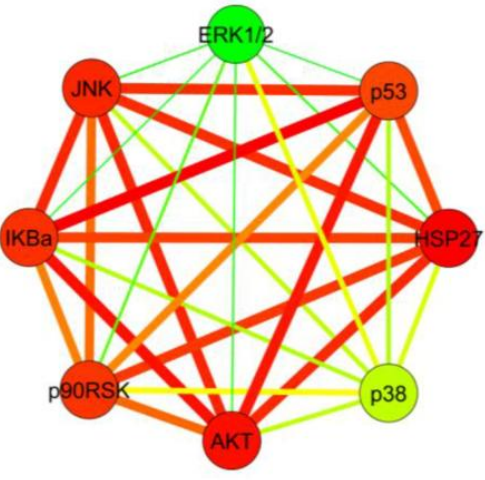

$10 \mu \mathrm{M}$ Deguelin + $34 \mathrm{nM}$ SB202474

Figure 3-5. Network graphs following exposure to a $10 \mu \mathrm{M}$ deguelin dose of deguelin alone, in combination with SB202190, and in combination with SB202474. Edge color and thickness are scaled to represent the Euclidean distance between proteins. Red thick lines correspond to short distances and green thin lines correspond to long distances. Node colors are scaled to represent the calculated radiality for each node. Nodes with high radiality values are shown in red and nodes with low radiality values are shown in green.

\section{Table. 3-2 Deguelin Alone Euclidean Distances}

\begin{tabular}{|c|c|c|c|c|c|c|c|c|c|c|c|c|c|c|c|c|c|}
\hline \multicolumn{3}{|c|}{$0.001 \mu \mathrm{M}$} & \multicolumn{3}{|l|}{$0.01 \mu \mathrm{M}$} & \multicolumn{3}{|l|}{$\underline{0.1 \mu \mathrm{M}}$} & \multicolumn{3}{|l|}{$1 \mu \mathrm{M}$} & \multicolumn{3}{|l|}{$10 \mu \mathrm{M}$} & \multicolumn{3}{|l|}{$100 \mu \mathrm{M}$} \\
\hline Node 1 & Node 2 & Distance & Node 1 & Node 2 & Distance & Node 1 & Node 2 & Distance & Node 1 & Node 2 & Distance & Node 1 & Node 2 & Distance & Node 1 & Node 2 & Distance \\
\hline p90RSK & ERK1/2 & 4.5 & p53 & HSP27 & 10.9 & $\mathrm{IKBa}$ & AKT & 18.2 & HSP27 & JNK & 1.5 & p53 & $\mathrm{IKBa}$ & 4.0 & p90RSK & AKT & 26.6 \\
\hline p53 & AKT & 12.8 & p90RSK & AKT & 11.2 & p90RSK & $\mathrm{IKBa}$ & 20.0 & $\mathrm{IKBa}$ & HSP27 & 13.3 & HSP27 & ERK1/2 & 11.2 & $\mathrm{IKBa}$ & AKT & 28.2 \\
\hline p53 & $\mathrm{IKBa}$ & 15.7 & p90RSK & $\mathrm{IKBa}$ & 11.4 & p90RSK & AKT & 22.6 & $\mathrm{IKBa}$ & JNK & 13.4 & p90RSK & AKT & 19.7 & p53 & $\mathrm{IKBa}$ & 30.2 \\
\hline $\mathrm{IKBa}$ & ERK1/2 & 23.3 & $\mathrm{IKBa}$ & AKT & 19.7 & p90RSK & ERK $1 / 2$ & 48.4 & p38 & ERK1/2 & 14.8 & $\mathrm{IKBa}$ & AKT & 27.4 & p90RSK & $\mathrm{IKBa}$ & 53.6 \\
\hline p90RSK & $\mathrm{IKBa}$ & 27.8 & ERK1/2 & AKT & 19.8 & $\mathrm{IKBa}$ & ERK1/2 & 61.2 & JNK & AKT & 30.3 & p53 & AKT & 27.6 & p53 & $\mathrm{AKT}$ & 56.6 \\
\hline $\mathrm{IKBa}$ & AKT & 28.5 & p90RSK & ERK1/2 & 30.9 & p53 & HSP27 & 66.2 & HSP27 & AKT & 31.8 & JNK & ERK1/2 & 37.4 & p90RSK & p53 & 79.2 \\
\hline p53 & ERK $1 / 2$ & 37.7 & $\mathrm{IKBa}$ & ERK1/2 & 39.0 & ERK1/2 & AKT & 70.9 & $\mathrm{IKBa}$ & AKT & 35.6 & HSP27 & JNK & 37.7 & p38 & ERK $1 / 2$ & 82.6 \\
\hline p90RSK & p53 & 42.1 & p53 & p38 & 61.6 & p53 & p38 & 79.8 & p53 & $\mathrm{IKBa}$ & 36.2 & p90RSK & $\mathrm{IKBa}$ & 46.3 & HSP27 & JNK & 92.2 \\
\hline ERK1/2 & AKT & 50.1 & HSP27 & p38 & 72.4 & p53 & AKT & 97.8 & p53 & HSP27 & 45.4 & p90RSK & p53 & 47.0 & p90RSK & JNK & 92.8 \\
\hline p90RSK & AKT & 54.4 & p90RSK & HSP27 & 83.0 & HSP27 & p38 & 112.3 & p53 & JNK & 46.4 & p90RSK & JNK & 55.5 & JNK & AKT & 107.1 \\
\hline p53 & HSP27 & 75.5 & $\mathrm{IKBa}$ & HSP27 & 84.3 & p53 & $\mathrm{IKBa}$ & 112.5 & p53 & AKT & 71.6 & p90RSK & ERK1/2 & 66.4 & HSP27 & ERK $1 / 2$ & 111.8 \\
\hline HSP27 & AKT & 76.7 & HSP27 & AKT & 92.4 & p90RSK & p53 & 119.5 & p90RSK & AKT & 120.6 & JNK & AKT & 67.8 & p90RSK & HSP27 & 124.5 \\
\hline $\mathrm{IKBa}$ & HSP27 & 77.0 & p90RSK & p53 & 93.1 & p38 & AKT & 129.0 & p90RSK & ERK1/2 & 144.7 & p90RSK & HSP27 & 75.2 & $\mathrm{IKBa}$ & JNK & 132.7 \\
\hline HSP27 & p38 & 93.0 & p53 & $\mathrm{IKBa}$ & 94.0 & $\mathrm{IKBa}$ & p38 & 134.2 & p90RSK & JNK & 150.4 & ERK1/2 & AKT & 84.7 & HSP27 & AKT & 150.1 \\
\hline HSP27 & ERK1/2 & 94.0 & p53 & AKT & 102.8 & p90RSK & p38 & 150.5 & p90RSK & HSP27 & 151.9 & p53 & JNK & 92.5 & p53 & JNK & 162.7 \\
\hline p90RSK & HSP27 & 97.7 & HSP27 & ERK1/2 & 108.1 & HSP27 & AKT & 163.1 & p90RSK & $\mathrm{IKBa}$ & 155.7 & HSP27 & AKT & 92.8 & $\mathrm{IKBa}$ & HSP27 & 178.0 \\
\hline IKBa & p38 & 162.4 & p53 & ERK1/2 & 118.8 & p53 & ERK $1 / 2$ & 167.7 & p90RSK & p38 & 159.3 & $\mathrm{IKBa}$ & JNK & 93.6 & JNK & ERK $1 / 2$ & 179.4 \\
\hline p53 & p38 & 165.2 & $\mathrm{IKBa}$ & p38 & 150.8 & $\mathrm{IKBa}$ & HSP27 & 178.5 & p90RSK & p53 & 190.5 & $\mathrm{IKBa}$ & ERK1/2 & 112.1 & HSP27 & p38 & 186.6 \\
\hline p38 & AKT & 168.6 & p90RSK & p38 & 152.0 & p90RSK & HSP27 & 184.0 & ERK1/2 & AKT & 265.0 & p53 & ERK1/2 & 112.2 & p53 & HSP27 & 203.0 \\
\hline p38 & ERK $1 / 2$ & 172.6 & p38 & $\mathrm{AKT}$ & 162.3 & p38 & ERK $1 / 2$ & 195.3 & p38 & AKT & 279.5 & p53 & HSP27 & 120.0 & p38 & JNK & 233.6 \\
\hline p90RSK & p38 & 175.1 & p38 & ERK1/2 & 179.3 & JNK & ERK $1 / 2$ & 214.5 & JNK & ERK1/2 & 294.6 & $\mathrm{IKBa}$ & HSP27 & 120.2 & p90RSK & ERK $1 / 2$ & 235.5 \\
\hline p90RSK & JNK & 314.8 & JNK & ERK $1 / 2$ & 289.6 & HSP27 & ERK1/2 & 231.7 & HSP27 & ERK1/2 & 296.0 & HSP27 & p38 & 312.6 & ERK $1 / 2$ & $\mathrm{AKT}$ & 260.5 \\
\hline JNK & ERK $1 / 2$ & 316.9 & JNK & AKT & 307.9 & p90RSK & JNK & 262.8 & $\mathrm{IKBa}$ & ERK1/2 & 300.3 & p38 & ERK1/2 & 320.2 & $\mathrm{IKBa}$ & ERK $1 / 2$ & 288.6 \\
\hline p53 & JNK & 327.0 & p90RSK & JNK & 318.9 & $\mathrm{IKBa}$ & JNK & 274.8 & p38 & JNK & 309.0 & p38 & JNK & 345.1 & p90RSK & p38 & 305.1 \\
\hline $\mathrm{IKBa}$ & JNK & 327.2 & $\mathrm{IKBa}$ & JNK & 323.1 & JNK & $\mathrm{AKT}$ & 285.3 & HSP27 & p38 & 310.4 & p90RSK & p38 & 386.2 & p53 & ERK1/2 & 314.5 \\
\hline JNK & AKT & 327.4 & HSP27 & JNK & 396.1 & p53 & JNK & 381.0 & $\mathrm{IKBa}$ & p38 & 314.9 & p38 & AKT & 404.8 & p38 & AKT & 328.2 \\
\hline HSP27 & JNK & 402.4 & p53 & JNK & 407.0 & p38 & JNK & 406.5 & p53 & ERK1/2 & 335.2 & $\mathrm{IKBa}$ & p38 & 432.2 & $\mathrm{IKBa}$ & p38 & 356.4 \\
\hline p38 & JNK & 489.0 & p38 & JNK & 468.3 & HSP27 & JNK & 442.6 & p53 & p38 & 349.8 & p53 & p38 & 432.3 & p53 & p38 & 384.1 \\
\hline
\end{tabular}


Normalized radiality parameters for p38, ERK1/2, and JNK responses to deguelin alone are depicted in Figure 3-6. Radiality concisely describes each protein's simultaneous phosphorylation mediated relation to all other proteins in the network, and was calculated as described in the methods section (above). Fluctuations in radiality values indicate that the inhibitor treatment is causing the network relationships between signaling proteins to change. Radiality remains relatively constant in the dosing range of 0.001 to $0.1 \mu \mathrm{M}$ for JNK, p38, and ERK1/2 MAPKs, suggesting that these proteins signaling activities are not altered in this dosing range. JNK radiality is lower than both p38 and ERK1/2 in the 0.001 to $0.1 \mu \mathrm{M}$ range. At doses of deguelin $1 \mu \mathrm{M}$ and above, the normalized radiality parameters for p38, ERK1/2, and JNK change: JNK radiality is increased while p38 and ERK1/2 radiality is decreased at the $1 \mu \mathrm{M}$ dose; JNK radiality remains high at the 10 and $100 \mu \mathrm{M}$ doses, while p38 radiality remains low at the 10 and $100 \mu \mathrm{M}$ doses. ERK1/2 radiality is increased at the $10 \mu \mathrm{M}$ dose to a level close to that of JNK, and is slightly decreased at the $100 \mu \mathrm{M}$ dose. The normalized radiality parameters for AKT, I $\kappa \mathrm{B} \alpha, \mathrm{p} 53$, p90RSK, and HSP27 remain relatively constant across the entire dosing range (Figure 3-7). 


\section{Deguelin}

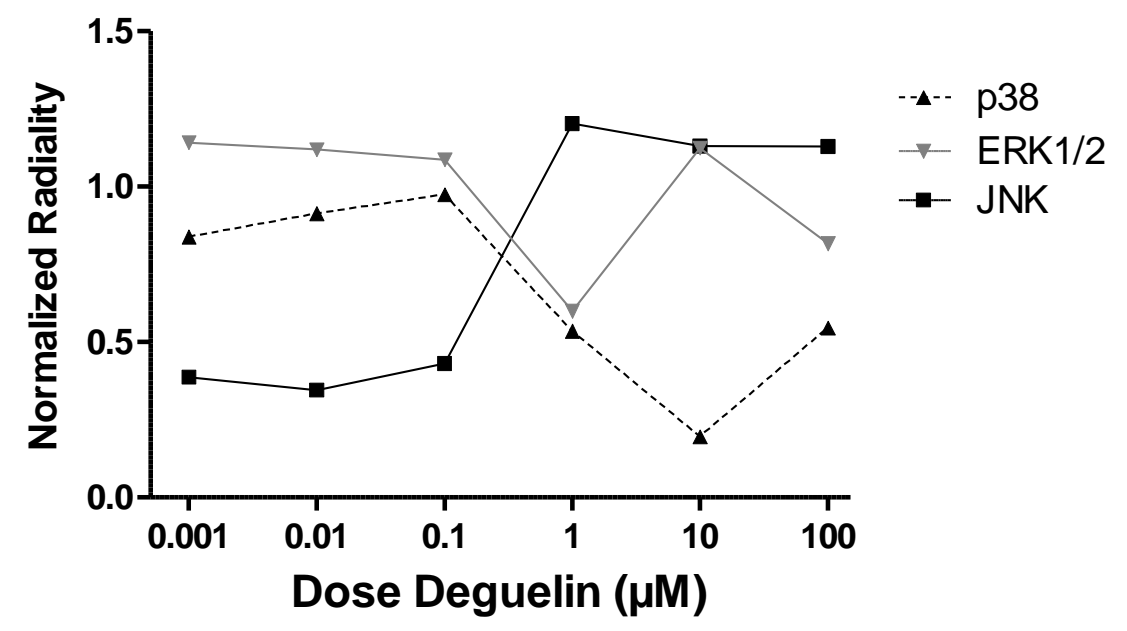

Figure 3-6. Normalized radiality dose-response curves for JNK, ERK1/2, and p38 responses following exposure to deguelin alone. Radiality parameters are normalized to the sum of radiality parameters for all measured proteins.

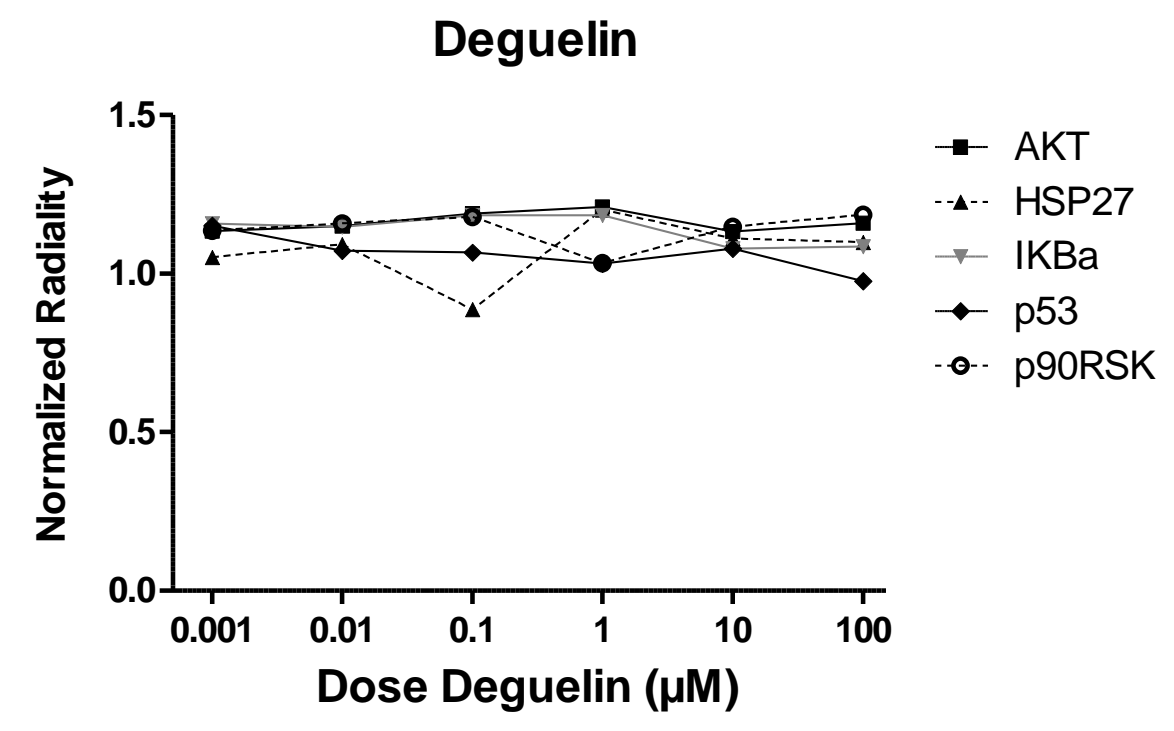

Figure 3-7: Normalized radiality dose-response curves for all other protein responses following exposure to deguelin alone. Radiality parameters are normalized to the sum of radiality parameters for all measured proteins. 


\subsubsection{Deguelin in Combination with SB202190.}

Cells were treated with SB202190 in combination with deguelin to further probe the alterations in p38, ERK1/2, and JNK network centrality observed in response to treatment with deguelin alone, as initial results show that SB202190 partially inhibits both p38 and JNK (Figure 3-3B). Table 3-3 shows the calculated distance values for each pair of proteins in response to treatment with deguelin in combination with SB202190. When cells were dosed with $350 \mathrm{nM}$ SB202190 in combination with the lower doses of deguelin $(0.001-0.1 \mu \mathrm{M})$ the p38-ERK1/2, JNK-ERK1/2, and p38-JNK distances were shorter in comparison to when cells were treated with deguelin alone. At the $1 \mu \mathrm{M}$ dose of deguelin, the distance between p38 and JNK was the shortest distance between any of the 3 MAPKs (JNK, p38, and ERK1/2). At the $10 \mu \mathrm{M}$ dose the distances between all three MAPKs increased. At the $100 \mu \mathrm{M}$ dose of deguelin, the distance between p38 and ERK1/2 was much shorter in comparison to the JNK-ERK1/2 and p38-JNK distances. 
Table. 3-3 Deguelin + 350 nM SB202190 Euclidean Distances

\begin{tabular}{|c|c|c|c|c|c|c|c|c|c|c|c|c|c|c|c|c|c|}
\hline \multicolumn{18}{|c|}{ Dose Deguelin } \\
\hline \multicolumn{3}{|c|}{$0.001 \mu \mathrm{M}$} & \multicolumn{3}{|l|}{$0.01 \mu \mathrm{M}$} & \multicolumn{3}{|l|}{$\underline{0.1 \mu \mathrm{M}}$} & \multicolumn{3}{|l|}{$1 \mu \mathrm{M}$} & \multicolumn{3}{|l|}{$10 \mu \mathrm{M}$} & \multicolumn{3}{|l|}{$100 \mu \mathrm{M}$} \\
\hline Node 1 & Node 2 & Distance & Node 1 & Node 2 & Distance & Node 1 & Node 2 & Distance & Node 1 & Node 2 & Distance & Node 1 & Node 2 & Distance & Node 1 & Node 2 & Distance \\
\hline p90RSK & JNK & 6.3 & p90RSK & AKT & 27.5 & p90RSK & HSP27 & 27.0 & JNK & AKT & 18.3 & p90RSK & HSP27 & 26.6 & p90RSK & $\mathrm{IKBa}$ & 16.5 \\
\hline $\mathrm{IKBa}$ & p38 & 39.2 & JNK & AKT & 27.7 & $\mathrm{IKBa}$ & p38 & 32.9 & HSP27 & JNK & 26.6 & p53 & $\mathrm{IKBa}$ & 27.8 & p90RSK & p53 & 29.4 \\
\hline $\mathrm{IKBa}$ & ERK $1 / 2$ & 53.2 & HSP27 & JNK & 33.8 & p53 & AKT & 49.9 & HSP27 & AKT & 42.8 & HSP27 & AKT & 38.6 & p53 & $\mathrm{IKBa}$ & 35.7 \\
\hline HSP27 & JNK & 66.4 & $\mathrm{IKBa}$ & p38 & 40.4 & p90RSK & JNK & 52.4 & HSP27 & p38 & 60.4 & p90RSK & AKT & 50.8 & $\mathrm{IKBa}$ & AKT & 38.2 \\
\hline p90RSK & HSP27 & 67.2 & p38 & ERK $1 / 2$ & 51.6 & HSP27 & JNK & 53.3 & p38 & JNK & 69.7 & p53 & AKT & 50.8 & p38 & ERK $1 / 2$ & 45.0 \\
\hline HSP27 & ERK $1 / 2$ & 67.4 & p90RSK & JNK & 55.1 & $\mathrm{IKBa}$ & ERK $1 / 2$ & 61.1 & p90RSK & ERK $1 / 2$ & 70.8 & p90RSK & p38 & 59.9 & p90RSK & AKT & 53.1 \\
\hline p38 & ERK $1 / 2$ & 91.8 & HSP27 & AKT & 60.0 & p38 & ERK $1 / 2$ & 80.1 & p38 & AKT & 70.9 & $\mathrm{IKBa}$ & JNK & 71.8 & p53 & AKT & 72.3 \\
\hline p90RSK & AKT & 95.5 & $\mathrm{IKBa}$ & ERK $1 / 2$ & 78.3 & JNK & ERK $1 / 2$ & 109.2 & p53 & ERK $1 / 2$ & 81.5 & HSP27 & p38 & 74.6 & HSP27 & AKT & 78.2 \\
\hline JNK & AKT & 99.8 & p90RSK & HSP27 & 86.2 & p90RSK & AKT & 113.7 & p90RSK & p53 & 87.2 & $\mathrm{IKBa}$ & AKT & 78.2 & p90RSK & JNK & 86.4 \\
\hline JNK & ERK $1 / 2$ & 104.3 & HSP27 & ERK $1 / 2$ & 119.4 & HSP27 & AKT & 129.0 & p38 & ERK1/2 & 93.6 & p53 & JNK & 83.0 & IKBa & HSP27 & 97.2 \\
\hline p90RSK & ERK $1 / 2$ & 108.8 & JNK & ERK $1 / 2$ & 134.8 & HSP27 & ERK $1 / 2$ & 138.5 & p53 & p38 & 111.0 & p53 & HSP27 & 86.5 & $\mathrm{IKBa}$ & JNK & 100.7 \\
\hline $\mathrm{IKBa}$ & HSP27 & 112.3 & ERK $1 / 2$ & AKT & 159.2 & p90RSK & p53 & 144.9 & ERK $1 / 2$ & AKT & 143.9 & p90RSK & p53 & 101.6 & p53 & JNK & 102.1 \\
\hline p53 & AKT & 137.3 & p90RSK & p53 & 163.8 & p90RSK & ERK $1 / 2$ & 153.9 & HSP27 & ERK1/2 & 151.4 & p38 & AKT & 109.3 & p53 & HSP27 & 112.5 \\
\hline HSP27 & p38 & 145.5 & HSP27 & p38 & 170.9 & $\mathrm{IKBa}$ & JNK & 163.8 & JNK & ERK $1 / 2$ & 151.8 & JNK & AKT & 111.9 & p90RSK & HSP27 & 112.9 \\
\hline HSP27 & AKT & 156.3 & p90RSK & ERK $1 / 2$ & 185.5 & JNK & $\mathrm{AKT}$ & 164.7 & p90RSK & p38 & 158.1 & $\mathrm{IKBa}$ & HSP27 & 114.2 & JNK & AKT & 125.1 \\
\hline $\mathrm{IKBa}$ & JNK & 157.4 & p38 & JNK & 185.5 & p53 & HSP27 & 165.6 & p53 & HSP27 & 167.8 & p90RSK & $\mathrm{IKBa}$ & 128.8 & HSP27 & JNK & 197.3 \\
\hline p90RSK & IKBa & 161.7 & p53 & AKT & 190.9 & p38 & JNK & 188.5 & p53 & JNK & 180.6 & HSP27 & JNK & 149.6 & HSP27 & p38 & 221.7 \\
\hline p38 & JNK & 195.4 & $\mathrm{IKBa}$ & HSP27 & 191.5 & p53 & JNK & 190.9 & p53 & AKT & 180.8 & p90RSK & JNK & 152.0 & p38 & AKT & 236.3 \\
\hline p90RSK & p38 & 199.4 & p38 & AKT & 209.1 & $\mathrm{IKBa}$ & HSP27 & 198.6 & p90RSK & AKT & 214.4 & p53 & p38 & 159.7 & HSP27 & ERK $1 / 2$ & 246.0 \\
\hline ERK1/2 & AKT & 204.1 & $\mathrm{IKBa}$ & JNK & 211.2 & p90RSK & $\mathrm{IKBa}$ & 212.1 & p90RSK & HSP27 & 218.1 & $\mathrm{IKBa}$ & p38 & 187.3 & ERK $1 / 2$ & AKT & 246.9 \\
\hline p90RSK & p53 & 232.4 & p53 & JNK & 217.9 & HSP27 & p38 & 217.8 & p90RSK & JNK & 221.3 & p38 & JNK & 210.8 & $\mathrm{IKBa}$ & p38 & 274.5 \\
\hline p53 & JNK & 237.0 & p90RSK & p38 & 234.7 & p90RSK & p38 & 234.0 & IKBa & JNK & 222.9 & p38 & ERK $1 / 2$ & 231.8 & $\mathrm{IKBa}$ & ERK1/2 & 284.8 \\
\hline $\mathrm{IKBa}$ & AKT & 257.1 & $\mathrm{IKBa}$ & AKT & 236.6 & ERK1/2 & AKT & 266.6 & $\mathrm{IKBa}$ & AKT & 227.2 & p90RSK & ERK $1 / 2$ & 289.9 & p90RSK & p38 & 289.0 \\
\hline p53 & HSP27 & 289.2 & p53 & HSP27 & 245.3 & p53 & ERK $1 / 2$ & 298.2 & $\mathrm{IKBa}$ & HSP27 & 234.8 & HSP27 & ERK $1 / 2$ & 306.3 & p90RSK & ERK $1 / 2$ & 298.0 \\
\hline p38 & AKT & 294.8 & p90RSK & IKBa & 263.3 & $\mathrm{IKBa}$ & AKT & 325.5 & $\mathrm{IKBa}$ & p38 & 292.1 & ERK $1 / 2$ & AKT & 340.4 & p53 & p38 & 307.0 \\
\hline p53 & ERK $1 / 2$ & 341.2 & p53 & ERK $1 / 2$ & 348.6 & p38 & AKT & 346.4 & $\mathrm{IKBa}$ & ERK $1 / 2$ & 370.6 & p53 & ERK $1 / 2$ & 391.1 & p53 & ERK $1 / 2$ & 319.2 \\
\hline p53 & $\mathrm{IKBa}$ & 394.0 & p53 & p38 & 396.6 & p53 & $\mathrm{IKBa}$ & 354.6 & p53 & $\mathrm{IKBa}$ & 402.3 & $\mathrm{IKBa}$ & ERK $1 / 2$ & 418.6 & JNK & ERK1/2 & 339.2 \\
\hline p53 & p38 & 431.2 & p53 & $\mathrm{IKBa}$ & 426.7 & p53 & p38 & 378.2 & p90RSK & $\mathrm{IKBa}$ & 441.3 & JNK & ERK $1 / 2$ & 431.9 & p38 & JNK & 340.6 \\
\hline
\end{tabular}

Figure 3-8 shows the normalized radiality dose-response curve for p38, ERK1/2, and JNK responses in cells treated with deguelin in combination with SB202190. The normalized radiality values observed for deguelin plus SB202190 were relatively constant at the lower doses of deguelin $(0.001-0.1 \mu \mathrm{M})$. ERK1/2 and JNK radiality values remained relatively constant (ERK1/2: $1.06-1.12$; JNK: 1.17.-1.20) from 0.001 to $1 \mu \mathrm{M}$ and decreased at 10 and $100 \mu \mathrm{M}$. At $10 \mu \mathrm{M}$ deguelin ERK1/2 radiality was 0.32 , and at $100 \mu \mathrm{M}$ it was 0.50 . JNK radiality was 0.95 and 0.91 at the 10 and $100 \mu \mathrm{M}$ doses, respectively. p38 radiality remained relatively constant (0.82-0.94) from 0.001 to $0.1 \mu \mathrm{M}$ deguelin with values less than both ERK1/2 and JNK. At the 1 $\mu \mathrm{M}$ dose of deguelin, $\mathrm{p} 38$ radiality increased to 1.17 , a value greater than both ERK1/2 and JNK 
(1.06 and 1.15, respectively). At the 10 and $100 \mu \mathrm{M}$ doses of deguelin, p38 radiality was 1.04 and 0.56, respectively. Figure 3-9 shows the normalized radiality dose-response curve for all other protein responses in cells treated with deguelin in combination with SB202190.

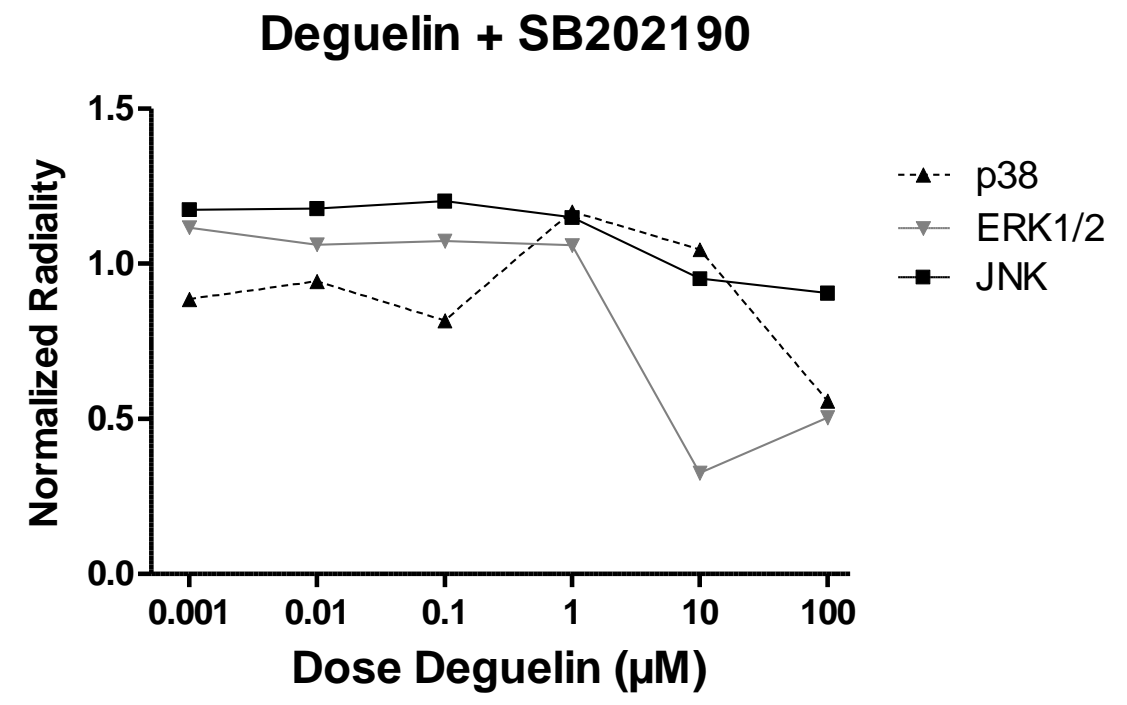

Figure 3-8. Normalized radiality dose-response curves for JNK, ERK1/2, and p38 responses following exposure to deguelin in combination with 350 nM SB202190. Radiality parameters are normalized to the sum of radiality parameters for all measured proteins.

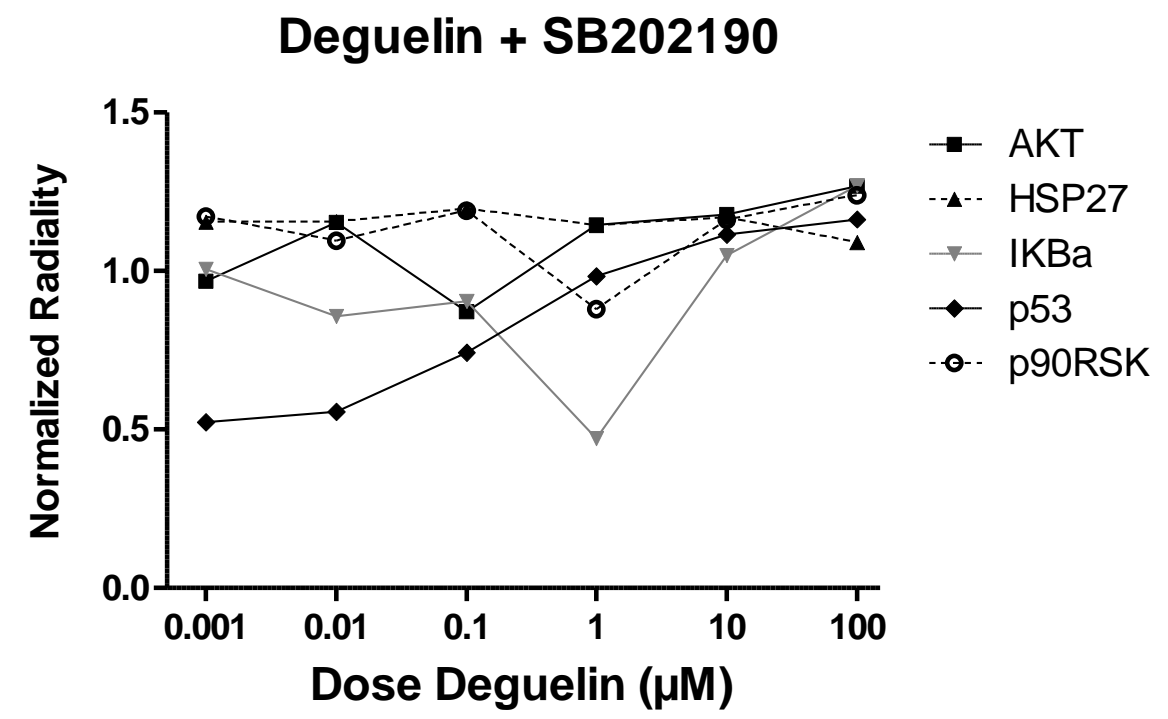

Figure 3-9. Normalized radiality dose-response curves for AKT, HSP27, IkB $\alpha, \mathrm{p} 53$, and p90RSK responses following exposure to deguelin in combination with 350 nM SB202190. Radiality parameters are normalized to the sum of radiality parameters for all measured proteins. 


\subsubsection{Deguelin in Combination with SB202474.}

Cells were treated with SB202474 in combination with deguelin to further probe the alterations in p38, ERK1/2, and JNK network centrality, as initial results show that SB202474 partially inhibits JNK, but not p38 (Figure 3-3B). Table 3-4 shows the distance values for each pair of proteins in response to treatment with deguelin in combination with SB202474. The distances between p38 and ERK1/2 were shorter than the JNK-ERK1/2 and p38-JNK distances for the $0.001,0.01$, and $0.1 \mu \mathrm{M}$ doses. The p38-ERK1/2 distance increased dramatically at the 1 $\mu \mathrm{M}$ dose of deguelin while the p38-JNK distance decreased dramatically. At the $1 \mu \mathrm{M}$ dose of deguelin, the p38-JNK distance was much less than the p38-ERK1/2 distance and the JNKERK1/2 distance. At the 10 and $100 \mu \mathrm{M}$ doses, the distances between all three MAPK's were relatively high. 
Table 3-4 Deguelin + 34 nM SB202474 Euclidean Distances

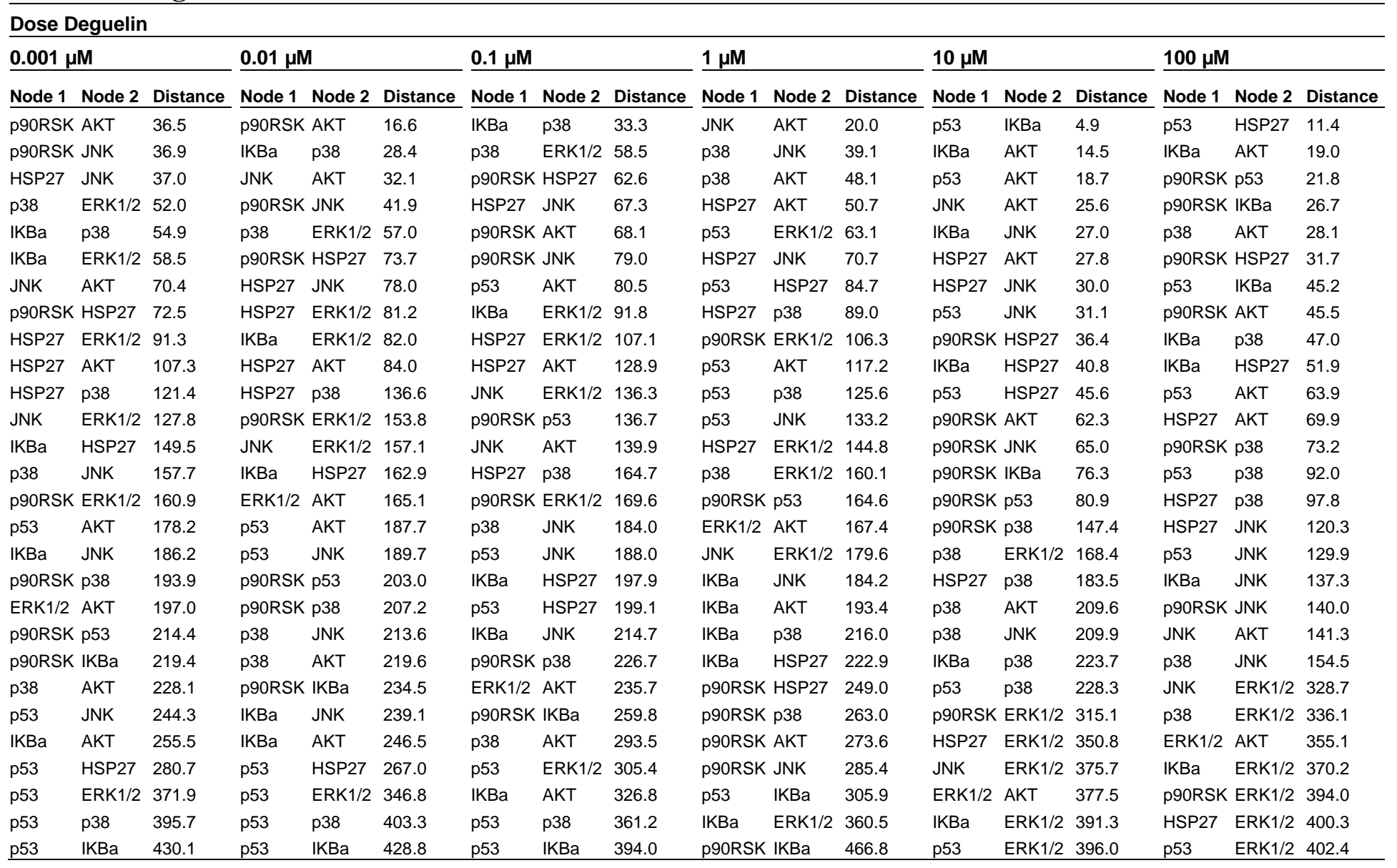

Figure 3-10 shows the normalized radiality dose-response curve for p38, ERK1/2, and JNK responses following treatment with deguelin in combination with SB202474. There is little change in the radiality values for any signaling proteins for the $0.001,0.01$, and $0.1 \mu \mathrm{M}$ doses of deguelin (JNK: 1.13-1.18; ERK1/2: 1.07-1.08; p38: 0.94-0.99). JNK radiality remains relatively constant all the way up to the $10 \mu \mathrm{M}$ dose. At the 10 and $100 \mu \mathrm{M}$ doses of deguelin JNK radiality was 1.15 and 0.93 , respectively. The $1 \mu \mathrm{M}$ dose of deguelin indicates a point of slight change for ERK1/2 and p38. At the 10 and $100 \mu \mathrm{M}$ doses of deguelin, ERK1/2 radiality decreased to 0.23 and 0.13 , respectively. p38 radiality was 0.81 at the $10 \mu \mathrm{M}$ dose of deguelin and 1.11 at the $100 \mu \mathrm{M}$ dose. Overall, the radiality curves for deguelin in combination with 
SB202474 are similar to the radiality curves for deguelin in combination with SB202190. The curves do differ in the p38 and ERK1/2 radiality parameters at the 10 and $100 \mu \mathrm{M}$ doses of deguelin. This coincides with the viability curves for the 2 treatments, showing that treatment with deguelin in combination with SB202474 leads to significantly decreased $(p<0.01)$ viability at the 10 and $100 \mu \mathrm{M}$ dose of deguelin while treatment with deguelin in combination with SB202190 does not lead to significantly decreased viability at any doses tested. Figure 3-11 shows the normalized radiality dose-response curve for all other protein responses in cells treated with deguelin in combination with SB202474.

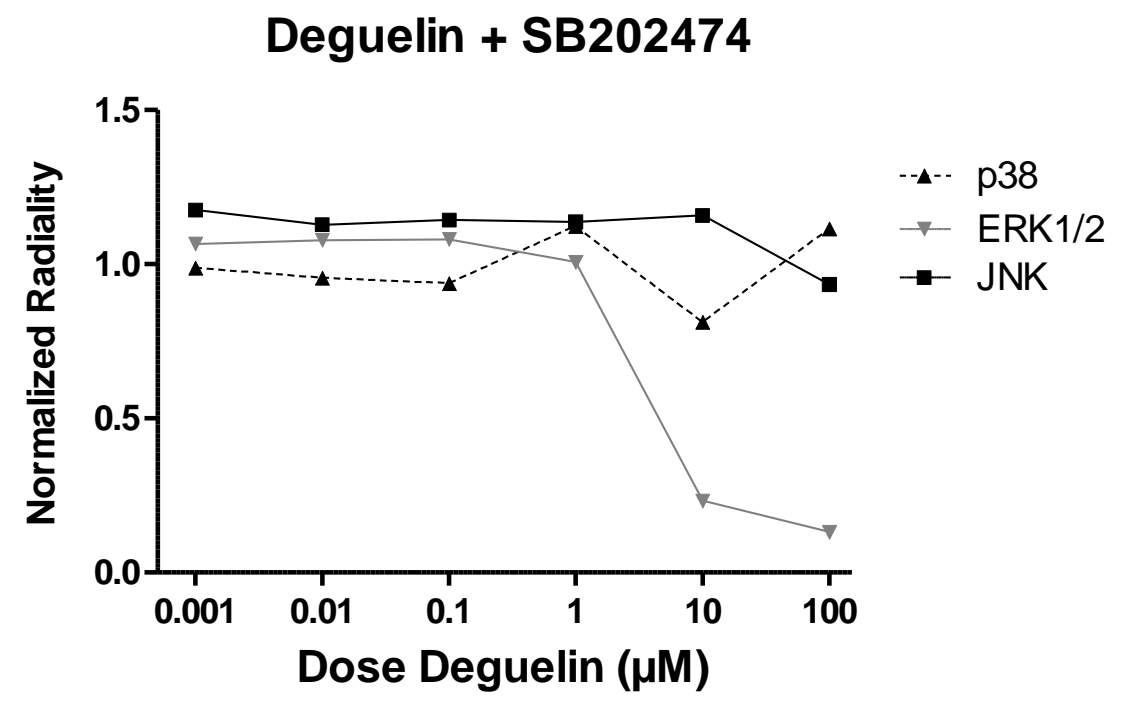

Figure 3-10. Normalized radiality dose-response curves for JNK, ERK1/2, and p38 responses following exposure to deguelin in combination with 34 nM SB202474. Radiality parameters are normalized to the sum of radiality parameters for all measured proteins. 


\section{Deguelin + SB202474}

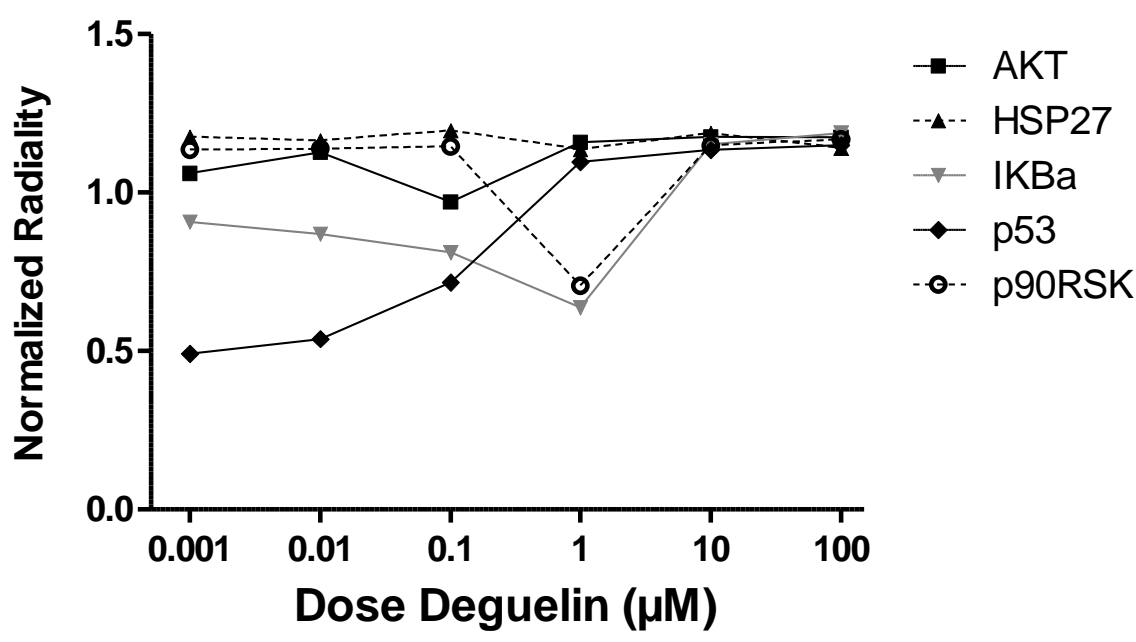

Figure 3-11. Normalized radiality dose-response curves for AKT, HSP27, IkB $\alpha$, p53, and p90RSK responses following exposure to deguelin in combination with 34 nM SB202474. Radiality parameters are normalized to the sum of radiality parameters for all measured proteins.

\subsection{Discussion}

With regard to toxicity testing, there is a current call to action for the modernization of approaches that are used to quantitatively assess the health risks associated with chemical exposure. $^{1,3,43}$ Specifically, there is support for the integration of pathway based toxicity assays into current methods to provide a better understanding of how even low-dose exposures perturb biological processes in human cells and tissues. ${ }^{4}$ The major focus of this modernization has been on monitoring the up regulation of proteins in specific pre-defined pathways. ${ }^{1,4,19,43-45}$ It is now understood that protein pathways are members of larger dynamic networks, that are highly interconnected, and not always linear. ${ }^{46}$ One way of integrating pathway-based methods of monitoring toxicity is to incorporate quantitative graph theory parameters that describe the relevance or centrality of proteins within the network framework following chemical exposure. Here, a simple data-driven approach was used to obtain such parameters, describing MAPK signaling network perturbations that occur following chemically induced mitochondrial stress. 
Importantly, network parameters were assessed at each individual inhibitor dose, which allows for a clear association between dose and perturbation response.

Graph theory was used to describe the balance between the traditionally distinct (i.e. linear) ERK1/2, p38, and JNK MAPK pathways. Dose-response curves were constructed for each protein where radiality was the response. Changes in the normalized radiality of MAPKs indicate a shift in the balance of these MAPK pathways. The dose-response curves allowed identification of shifts in individual protein centralities that accompany toxic exposures. This approach is advantageous in comparison to other methods that investigate signaling, because it is capable of revealing network perturbations that are induced by individual doses. An additional advantage of this approach is that centrality parameters can be calculated based on weighted edge values rather than the binary existence or absence of an edge that is typical of network analyses, eliminating the need for set threshold values.

The results provide an integrated view of how low doses of deguelin perturb normal MAPK signaling, and show that between the 0.1 and $1 \mu \mathrm{M}$ doses of deguelin, there exists a likely threshold dose where perturbation occurs. This is easily visualized by the dose-response curves for p38, ERK1/2, and JNK radiality following exposure to deguelin (Figure 3-6). The MAPK centralities remain relatively constant for doses lower than $1 \mu \mathrm{M}$, but at doses $1 \mu \mathrm{M}$ and higher there is a switch where JNK radiality becomes higher, p38 radiality decreases, and ERK1/2 radiality varies. The ranked order of $\mathrm{p} 38, \mathrm{JNK}$, and ERK1/2 radiality values remains the same for the $0.001,0.01$, and $0.1 \mu \mathrm{M}$ doses of deguelin, indicating that these doses do not lead to changes in the relationships between the signaling proteins. The observed trends in the dose-response radiality curves provide "snap shots" of signaling pathway alterations that occur when cells perceive that an applied stress has reached a level in which a change in the 
mechanism of response to the stress is necessary. For all proteins other than ERK1/2, JNK, and p38, radiality remained relatively constant across the entire dosing range (Figure 3-7), thus supporting the concept of this type of analysis for the identification of perturbations to normal network signaling. It is important to note that none of the doses of deguelin tested significantly reduced HepG2 viability after 400 min of exposure (Figure 3-4A). Exposure to deguelin for $24 \mathrm{~h}$ at doses higher than $1 \mu \mathrm{M}$ does however lead to significantly decreased HepG2 viability. Therefore, the analysis of the 400 min data revealed alterations in network signaling induced by deguelin at a point where toxicity as determined by the MTT assay was not yet observed. This demonstrates the utility of such data driven methods for assessing biological effects on a dose-todose basis, and determining significant doses capable of altering network signaling before viability is compromised. The critical doses identified in this study may capture points where cells are teetering between initiating death and promoting survival, and the application of graph theory helps to illuminate shifts in the balance between MAPK pathways.

SB202190 and SB202474 were selected as secondary inhibitors (dosed in combination with deguelin) to further investigate the balance between the MAPK signaling pathways following chemically induced stress. Figure 3-3B confirms that SB202190 and SB20474 exert the same effects on all of the proteins studied, except p38. Both compounds inhibit JNK, thus allowing comparison of partial JNK inhibition to partial JNK and p38 inhibition during deguelin exposure. Comparison of the Euclidean distances and radiality parameters obtained for cells treated with deguelin in combination with SB202190 (Table 3-3) to cells treated with deguelin in combination with SB202474 (Table 3-4) show similar results up to the $1 \mu \mathrm{M}$ dose of deguelin. Under both sets of conditions JNK radiality remains relatively high across the entire dosing range (Figure 3-8 and Figure 3-10). At the 10 and $100 \mu \mathrm{M}$ doses of deguelin, partial inhibition 
of p38 by SB202190 leads to a decrease in p38 radiality while SB202474 does not have this same effect. ERK1/2 radiality decreases for both sets of conditions at these doses, but more so with deguelin in combination with SB202474. The only significant decreases in viability observed for any of the treatments in this study were observed for the 10 and $100 \mu \mathrm{M}$ doses of deguelin in combination with SB202474 (Figure 3-4A). Comparison of the dose-response radiality curves for all three treatments (Figure 3-6, Figure 3-8, and Figure 3-10) shows that the unique feature for deguelin in combination with SB202474 is that ERK1/2 radiality is extremely low (effectively removed from the network) while p38 radiality is high. Treatment with deguelin alone, or in combination with SB202190, leads to decreased radiality for p38 and comparatively higher radiality values for ERK1/2. This indicates that for cellular viability to be decreased in the cells tested; both p38 and JNK must maintain a central role within the network, while ERK1/2's centrality is simultaneously diminished. ERK1/2 has previously been shown to promote survival under conditions of stress when apoptosis is not favorable. ${ }^{47-49}$ When apoptosis is favorable, both JNK and p38 signaling suppress the survival promoting activity of ERK1/2 via crosstalk, thereby inducing apoptosis. ${ }^{15,} 18,50-52$ The dynamic balance between these three pathways has been shown to be a key determinant as to whether cells survive a threat or die. ${ }^{9,15}$, 18 Specifically, the sustained activation of JNK and p38 signaling along with ERK1/2 pathway inhibition has been shown to be required for the induction of apoptosis, ${ }^{18}$ and the results of this study clearly support this notion. In addition to allowing the visualization of p38, ERK1/2 and JNK signaling dynamics, the analysis also captured the p53 response to stress; p53 radiality increases in a dose dependent manner in cells exposed to deguelin in combination with SB202190 or SB202474 (Figure 3-9 and Figure 3-11, respectively). It is well known that p53 is activated following a variety of cellular stresses playing a pivotal role in the stress response, ${ }^{53}$ 
which provides further validity to the proposed analysis approach to characterize networked cellular responses.

\subsection{Conclusions}

In agreement with previous studies, the results show that crosstalk between p38, ERK1/2, and JNK signaling pathways is a complex and dynamic process. ${ }^{12,15,54,55}$ The approach presented was able to extract information reflecting the dynamic nature of MAPK signaling and the central influence that the balance between ERK1/2, JNK, and p38 signaling crosstalk has on whether or not cells maintain viability following chemical insult. Simple inspection of the relative phosphorylation responses to each set of inhibitor treatments in Figure 3-2, as well as the significant differences between these responses in Table 3-1, cannot provide such an integrated description, thus demonstrating the utility of graph theory based methods for assessing toxicological actions more broadly by reflecting a networked response. The work presented here is well suited for identifying xenobiotic doses that induce changes in protein networks before typical apical toxicological endpoints are reached. The calculated graph theory parameters provide an integrated way of describing the degree of perturbation induced by specific doses of toxic compounds in an in vitro human model by providing an informative and sensitive means of assessing chemical effects on biological systems. These types of approaches have the ability to highlight low-dose exposure induced changes at the molecular level, and may provide an enhanced understanding of toxicological actions. In the future, the method presented in this study could be expanded to include multiple exposure times, in addition to multiple doses. The

analysis of network changes over a sequential time series could also provide a means to monitor network responses to xenobiotic exposures that contribute to adaptation. Additionally, network 
responses that lead to recovery/adaptation could be compared to responses that lead to death with this approach. This along with an increased number of protein targets could provide a rich depiction of the cells response to xenobiotic exposure, and represents a potential enhancement to current methods used to study toxic mechanisms of action. 


\subsection{Bibliography}

(1) Collins, F. S., Gray, G. M., and Bucher, J. R. (2008) Toxicology. Transforming environmental health protection. Science, 319, 906-907.

(2) Andersen, M. E., and Dennison, J. E. (2004) Mechanistic approaches for mixture risk assessments - present capabilities with simple mixtures and future directions., 16, 1-11.

(3) Bhattacharya, S., Zhang, Q., Carmichael, P. L., Boekelheide, K., and Andersen, M. E. (2011) Toxicity testing in the 21st century: defining new risk assessment approaches based on perturbation of intracellular toxicity pathways. PLoS One, 6, e20887.

(4) Judson, R. S., Kavlock, R. J., Setzer, R. W., Hubal, E. A., Martin, M. T., Knudsen, T. B., Houck, K. A., Thomas, R. S., Wetmore, B. A., and Dix, D. J. (2011) Estimating toxicity-related biological pathway altering doses for high-throughput chemical risk assessment. Chem Res Toxicol, 24, 451-462.

(5) Kong, A. N., Yu, R., Lei, W., Mandlekar, S., Tan, T. H., and Ucker, D. S. (1998) Differential activation of MAPK and ICE/Ced-3 protease in chemical-induced apoptosis. The role of oxidative stress in the regulation of mitogen-activated protein kinases (MAPKs) leading to gene expression and survival or activation of caspases leading to apoptosis. Restor Neurol Neurosci, 12, 63-70.

(6) Kultz, D. (2005) Molecular and evolutionary basis of the cellular stress response. Annu Rev Physiol, 67, 225-257.

(7) Kong, A. N., Yu, R., Chen, C., Mandlekar, S., and Primiano, T. (2000) Signal transduction events elicited by natural products: role of MAPK and caspase pathways in homeostatic response and induction of apoptosis. Arch Pharm Res, 23, 1-16.

(8) Owuor, E. D., and Kong, A. N. (2002) Antioxidants and oxidants regulated signal transduction pathways. Biochem Pharmacol, 64, 765-770.

(9) Matsuzawa, A., and Ichijo, H. (2005) Stress-responsive protein kinases in redox-regulated apoptosis signaling. Antioxid Redox Signal, 7, 472-481. 
(10) Cargnello, M., and Roux, P. P. (2011) Activation and Function of the MAPKs and Their Substrates, the MAPK-Activated Protein Kinases. Microbiol Mol Biol Rev, 75, 50-83.

(11) Singh, R. P., Dhawan, P., Golden, C., Kapoor, G. S., and Mehta, K. D. (1999) One-way cross-talk between $\mathrm{p38}(\mathrm{MAPK})$ and $\mathrm{p} 42 / 44(\mathrm{MAPK})$. Inhibition of $\mathrm{p38}$ (MAPK) induces low density lipoprotein receptor expression through activation of the p42/44(MAPK) cascade. J Biol Chem, 274, 19593-19600.

(12) Shen, Y. H., Godlewski, J., Zhu, J., Sathyanarayana, P., Leaner, V., Birrer, M. J., Rana, A., and Tzivion, G. (2003) Cross-talk between JNK/SAPK and ERK/MAPK pathways: sustained activation of JNK blocks ERK activation by mitogenic factors. J Biol Chem, 278, 26715-26721.

(13) Xiao, Y. Q., Malcolm, K., Worthen, G. S., Gardai, S., Schiemann, W. P., Fadok, V. A., Bratton, D. L., and Henson, P. M. (2002) Cross-talk between ERK and p38 MAPK mediates selective suppression of pro-inflammatory cytokines by transforming growth factor-beta. J Biol Chem, 277, 1488414893.

(14) Noselli, S., and Perrimon, N. (2000) Signal transduction. Are there close encounters between signaling pathways? Science, 290, 68-69.

(15) Junttila, M. R., Li, S.-P., and Westermarck, J. (2008) Phosphatase-mediated crosstalk between MAPK signaling pathways in the regulation of cell survival. FASEB J, 22, 954-965.

(16) Zhang, H., Shi, X., Hampong, M., Blanis, L., and Pelech, S. (2001) Stress-induced inhibition of ERK1 and ERK2 by direct interaction with p38 MAP kinase. J Biol Chem, 276, 6905-6908.

(17) Wang, Z., Yang, H., Tachado, S. D., Capó-Aponte, J. E., Bildin, V. N., Koziel, H., and Reinach, P. S. (2006) Phosphatase-Mediated Crosstalk Control of ERK and p38 MAPK Signaling in Corneal Epithelial Cells. Invest Ophthalmol Vis Sci, 47, 5267-5275.

(18) Xia, Z., Dickens, M., Raingeaud, J., Davis, R. J., and Greenberg, M. E. (1995) Opposing effects of ERK and JNK-p38 MAP kinases on apoptosis. Science, 270, 1326-1331. 
(19) Bhattacharya, S., Zhang, Q., Carmichael, P. L., Boekelheide, K., and Andersen, M. E. (2011) Toxicity testing in the 21 century: defining new risk assessment approaches based on perturbation of intracellular toxicity pathways. PLoS One, 6, e20887.

(20) Kim, W. Y., Chang, D. J., Hennessy, B., Kang, H. J., Yoo, J., Han, S. H., Kim, Y. S., Park, H. J., Seo, S. Y., Mills, G., Kim, K. W., Hong, W. K., Suh, Y. G., and Lee, H. Y. (2008) A novel derivative of the natural agent deguelin for cancer chemoprevention and therapy. Cancer Prev Res (Phila), 1, 577 587.

(21) Currie, H. N., Vrana, J. A., Han, A. A., Scardoni, G., Boggs, N., and Boyd, J. (2014) An approach to investigate intracellular protein network responses. Chem Res Tox, 27, 17-26.

(22) Okun, J. G., Lümmen, P., and Brandt, U. (1999) Three Classes of Inhibitors Share a Common Binding Domain in Mitochondrial Complex I (NADH:Ubiquinone Oxidoreductase). J Biol Chem, $274,2625-2630$.

(23) Murai, M., Ishihara, A., Nishioka, T., Takao Yagi, a., and Miyoshi, H. (2007) The ND1 Subunit Constructs the Inhibitor Binding Domain in Bovine Heart Mitochondrial Complex I. Biochemistry, $46,6409-6416$.

(24) Schuler, F., and Casida, J. E. (2001) Functional coupling of PSST and ND1 subunits in NADH:ubiquinone oxidoreductase established by photoaffinity labeling. Biochim Biophys Acta, $1506,79-87$.

(25) Degli Esposti, M. (1998) Inhibitors of NADH-ubiquinone reductase: an overview. Biochim Biophys Acta, $1364,222-235$.

(26) Degli Esposti, M., Ghelli, A., Ratta, M., Cortes, D., and Estornell, E. (1994) Natural substances (acetogenins) from the family Annonaceae are powerful inhibitors of mitochondrial NADH dehydrogenase (Complex I). Biochem J, 301, 161-167. 
(27) Brand, M. D., and Nicholls, D. G. (2011) Assessing mitochondrial dysfunction in cells. Biochem J, $435,297-312$.

(28) Gogvadze, V., Orrenius, S., and Zhivotovsky, B. (2008) Mitochondria in cancer cells: what is so special about them? Trends Cell Biol, 18, 165-173.

(29) Modica-Napolitano, J. S., and Singh, K. K. (2002) Mitochondria as targets for detection and treatment of cancer. Expert Rev Mol Med, 4, 1-19.

(30) Kroemer, G. (2006) Mitochondria in cancer. Oncogene, 25, 4630-4632.

(31) Boyd, J., Saksena, A., Patrone, J. B., Williams, H. N., Boggs, N., Le, H., and Theodore, M. (2011) Exploring the boundaries of additivity: mixtures of NADH: quinone oxidoreductase inhibitors. Chem Res Toxicol, 24, 1242-1250.

(32) Schmidt, C. W. (2010) Unraveling Environmental Effects on Mitochondria. Environ Health Perspect, 118, A292-A297.

(33) Scatena, R., Bottoni, P., Botta, G., Martorana, G. E., and Giardina, B. (2007) The role of mitochondria in pharmacotoxicology: a reevaluation of an old, newly emerging topic. Am J Physiol Cell Physiol, 293, C12-21.

(34) Sherer, T. B., Richardson, J. R., Testa, C. M., Seo, B. B., Panov, A. V., Yagi, T., Matsuno-Yagi, A., Miller, G. W., and Greenamyre, J. T. (2007) Mechanism of toxicity of pesticides acting at complex I: relevance to environmental etiologies of Parkinson's disease. J Neurochem, 100, 1469-1479.

(35) Green, D. R., and Kroemer, G. (2004) The Pathophysiology of Mitochondrial Cell Death. Science, $305,626-629$.

(36) Wallace, K. B., and Starkov, A. A. (2000) Mitochondrial targets of drug toxicity. Annu Rev Pharmacol Toxicol, 40, 353-388.

(37) Boyd, J., Vrana, J. A., and Williams, H. N. (2012) In vitro approach to predict post-translational phosphorylation response to mixtures. Toxicology, 313, 113-121. 
(38) Scardoni, G., and Laudanna, C. (2012) Centralities Based Analysis of Complex Networks. New Frontiers in Graph Theory, 323-348. Dr. Yagang Zhang (Ed.), InTech

(39) Borgatti, S. P., and Everett, M. G. (2006) A Graph-theoretic perspective on centrality. Social Networks, 28, 466-484.

(40) Lee, J. C., Laydon, J. T., McDonnell, P. C., Gallagher, T. F., Kumar, S., Green, D., McNulty, D., Blumenthal, M. J., Keys, J. R., Land vatter, S. W., Strickler, J. E., McLaughlin, M. M., Siemens, I. R., Fisher, S. M., Livi, G. P., White, J. R., Adams, J. L., and Young, P. R. (1994) A protein kinase involved in the regulation of inflammatory cytokine biosynthesis. Nature, 372, 739-746.

(41) Ho, A. K., Price, L., Mackova, M., and Chik, C. L. (2001) Potentiation of cyclic AMP and cyclic GMP accumulation by p38 mitogen-activated protein kinase (p38MAPK) inhibitors in rat pinealocytes. Biochem Pharmacol, 62, 1605-1611.

(42) Karahashi, H., Nagata, K., Ishii, K., and Amano, F. (2000) A selective inhibitor of p38 MAP kinase, SB202190, induced apoptotic cell death of a lipopolysaccharide-treated macrophage-like cell line, J774.1. Biochim Biophys Acta, 1502, 207-223.

(43) Krewski, D., Acosta, D., Jr., Andersen, M., Anderson, H., Bailar, J. C., 3rd, Boekelheide, K., Brent, R., Charnley, G., Cheung, V. G., Green, S., Jr., Kelsey, K. T., Kerkvliet, N. I., Li, A. A., McCray, L., Meyer, O., Patterson, R. D., Pennie, W., Scala, R. A., Solomon, G. M., Stephens, M., Yager, J., and Zeise, L. (2010) Toxicity testing in the 21st century: a vision and a strategy. J Toxicol Environ Health B Crit Rev, 13, 51-138.

(44) NRC. (2007) Toxicity Testing in the 21st Century: A Vision and a Strategy., Washington, DC.

(45) Simmons, S. O., Fan, C. Y., and Ramabhadran, R. (2009) Cellular stress response pathway system as a sentinel ensemble in toxicological screening. Toxicol Sci, 111, 202-225.

(46) Albert, R. (2005) Scale-free networks in cell biology. J Cell Sci, 118, 4947-4957. 
(47) Blank, J. L., Gerwins, P., Elliott, E. M., Sather, S., and Johnson, G. L. (1996) Molecular cloning of mitogen-activated protein/ERK kinase kinases (MEKK) 2 and 3. Regulation of sequential phosphorylation pathways involving mitogen-activated protein kinase and c-Jun kinase. J Biol Chem, 271, 5361-5368.

(48) Karandikar, M., Xu, S., and Cobb, M. H. (2000) MEKK1 binds raf-1 and the ERK2 cascade components. J Biol Chem, 275, 40120-40127.

(49) Waetzig, V., and Herdegen, T. (2005) MEKK1 controls neurite regrowth after experimental injury by balancing ERK1/2 and JNK2 signaling. Mol Cell Neurosci, 30, 67-78.

(50) Junttila, M. R., Ala-aho, R., Jokilehto, T., Peltonen, J., Kallajoki, M., Grenman, R., Jaakkola, P., and Westermarck, J. (2007) p38alpha and p38delta mitogen-activated protein kinase isoforms regulate invasion and growth of head and neck squamous carcinoma cells. Oncogene, 26, 52675279.

(51) Li, S. P., Junttila, M. R., Han, J., Kahari, V. M., and Westermarck, J. (2003) p38 Mitogen-activated protein kinase pathway suppresses cell survival by inducing dephosphorylation of mitogenactivated protein/extracellular signal-regulated kinase kinase1,2. Cancer Res, 63, 3473-3477.

(52) Liu, Q., and Hofmann, P. A. (2004) Protein phosphatase 2A-mediated cross-talk between p38 MAPK and ERK in apoptosis of cardiac myocytes. Am J Physiol Heart Circ Physiol, 286, H22042212.

(53) Murray-Zmijewski, F., Slee, E. A., and Lu, X. (2008) A complex barcode underlies the heterogeneous response of p53 to stress. Nat Rev Mol Cell Biol, 9, 702-712.

(54) Black, E. J., Walker, M., Clark, W., MacLaren, A., and Gillespie, D. A. (2002) Cell transformation by v-Jun deactivates ERK MAP kinase signalling. Oncogene, 21, 6540-6548.

(55) Friedman, A., and Perrimon, N. (2006) A functional RNAi screen for regulators of receptor tyrosine kinase and ERK signalling. Nature, 444, 230-234. 


\section{Chapter 4}

Tissue Trauma and Wound Healing Signaling Networks 


\subsection{Introduction}

In previous chapters, the network analysis approach developed in this body of work was focused on investigating intracellular signaling. In this chapter the boundaries of the studied network are expanded to encompass both extracellular and intracellular signaling providing a wider biological scope. The analysis was used to investigate the networked response of muscle tissue to traumatic injury. The tissue response to traumatic injury is extremely complex, involving coordinated efforts of many intracellular and extracellular proteins. Understanding this response is a difficult task, and one that requires the use of approaches capable of integrating multiple individual protein responses into easily interpreted biological meaning that describes their collective functioning as a network.

In this chapter, signaling networks were investigated in a rat model of traumatic injury where rats were subjected to a femur fracture. A dynamic network consisting of a wide variety of signaling molecules regulates the muscle healing process. It is known that proteins within networks that regulate the regeneration of muscle are spatiotemporally induced or repressed following muscle injury. ${ }^{l} \quad$ Yet, current understanding with regard to spatial differences in molecular responses to tissue injury (especially networked responses) is limited, and so the investigation was performed on rat muscle tissue samples collected from 3 different spatial locations with regard to the injury site. As an initial investigation, spatial comparisons between the levels of both extracellular and intracellular proteins at the 3 locations were made, and proteins displaying spatial concentration relationships to the injury site were identified. The presented network analysis approach was then used to describe the networked relationships of the signaling proteins at each location. Cytokines were chosen as extracellular signaling proteins to investigate due to their known roles as regulators of injury responses and inflammation. Several intracellular proteins including MAPKs were also investigated. 


\subsubsection{Cytokines}

Cytokines are low molecular weight water-soluble signaling proteins that are secreted by cells during inflammatory responses. ${ }^{2,3}$ Once secreted, cytokines bind to specific receptors on target cells to bring about change in the cell's function. Cytokines can affect nearly any biological process, and all cells, with the exception of red blood cells, can both produce and respond to cytokines. ${ }^{4}$ Cytokine actions can be autocrine, paracrine, or endocrince by acting on the cells that secreted them, on nearby cells, or on distant cells, respectively. Cytokines are produced as needed, and their mRNAs are short lived. ${ }^{5}$ Pleiotropism is a hallmark of cytokines; which have extremely variable and often contradictory activities ranging from inducing the production of inflammatory mediators (e.g. ROS, nitric oxide, prostaglandins, and other cytokines), cell recruitment, promotion of coagulant activity, assistance in antigen presentation, initiating cell death, or inducing growth and repair. ${ }^{6}$ Following inflammation inducing events such as infection or traumatic injury, an assortment of different cytokines are released by a variety of cell types. Each cytokine binds to a specific cell surface receptor initiating intracellular signaling cascades (including MAPK signaling) that can affect gene expression. ${ }^{4}$ Some cytokines can also act as transcription factors that bind directly to DNA or function as integral membrane proteins. ${ }^{4}$ Over the past 30 years cytokines have been investigated as diagnostic, prognostic, and therapeutic agents with some success, ${ }^{4}$ but the ways in which these diverse factors regulate complex cellular behavior remains poorly understood.

All inflammatory responses involve the production of a myriad of cytokines that can last for periods of hours to weeks. Differing patterns of cytokine expression provide different gene products responsible for specific cellular responses. ${ }^{5}$ Multiple cytokines can influence the same signaling pathways. Some cytokines can signal through multiple receptors, and sometimes 
different cytokines can both act through the same receptor. ${ }^{7}$ The pleiotropic nature of individual cytokines, overlapping actions of different cytokines, multiple cell types involved in cytokine signaling, and the many different signaling pathways that emanate from single cytokine receptors makes it difficult to understand how cytokines tailor their signaling to produce specific desired responses. ${ }^{5,8}$ Cytokines are potent, have high affinities for their receptors, and their receptors are expressed in very low numbers per cell. ${ }^{2}$ It is likely that the number of different cytokine receptors on a cell and the connectivity of its internal signaling networks are regulatory mechanisms that contribute to the variable responses induced by cytokines. Different cell types may respond differently to the same cytokine. Cytokines are important players in signaling networks that span multiple cells making them essential to cell to cell communication under periods of stress and injury. The diversity in cytokine signaling makes it difficult to understand how cells and the tissues they make up function as dynamic networks capable of appropriately responding to various environmental stresses.

\subsubsection{Tissue Trauma, Wound Healing, and Cytokines}

Cytokines act to promote tissue repair and homeostasis under periods of stress or injury. ${ }^{9}$ Wound healing is an evolutionarily conserved, sophisticated process involving the coordinated effort of several cell types. The stages of wound healing include inflammation, formation of new connective tissue and blood vessels, reepithelialization, matrix formation, and tissue

remodeling. ${ }^{10}$ This complex process is executed and regulated by the spatiotemporal actions of cytokines and the complex signaling networks they form through their receptors and downstream intracellular proteins. $^{9-11}$

Cytokines may have local effects or initiate systemic changes following injury. 3,12 Immediately following tissue injury the local production of cytokines increases drastically. 
Cytokines are produced by both infiltrating and resident cells. ${ }^{13}$ Injured cells release cytokines to achieve various outcomes including the recruitment of other cell types to the injury location. ${ }^{10,}$ ${ }^{14}$ Cells such as keratinocytes, firbroblasts, endothelial cells, macrophages, and platelets migrate and infiltrate the wound area where they proceed to proliferate and differentiate aiding in the removal of bacteria and tissue debri, the formation of new tissue, and the closing of the wound. ${ }^{10}$, 11, 14 Some cytokines serve as growth factors promoting the proliferation and differentiation of cells. Numerous studies have shown that the tissue response to injury involves variable fluctuations in heterogeneous populations of cytokines in both space and time. ${ }^{3,10,12,14-25}$ The success of wound healing depends on the complex integration of the extracellular signal inputs from cytokines. ${ }^{9} 10$ How these variations in cytokine concentrations cause cells to respond in a specifically regulated manner to carry out the wound healing process remains to be elucidated.

\subsubsection{Muscle Tissue Injury}

Skeletal muscle plays a central role in both motility and whole-body metabolism. Skeletal muscle is the most abundant tissue in the human body, ${ }^{26}$ and is primarily composed of elongated, parallel-running, multinucleated contractile muscle cells referred to as myofibers. ${ }^{27}$ Myofibers are heterogeneous with respect to size, metabolism, and contractile function. ${ }^{26,28}$ Uninjured postnatal muscle cells are remarkably adaptable, constantly sensing and responding to changes in the metabolic and functional demands of the organism. ${ }^{16}$ The number of myofibers can increase or decrease throughout life with muscle growth, hypertrophy, or atrophy. ${ }^{27}$ In response to physiological and pathological signals, muscle tissue remodels the biochemical, morphological, and physiological states of individual muscle cells through the activation of multiple intracellular signaling pathways that culminate in the activation or repression of a 
myriad of genes. ${ }^{16}$ The plasticity of muscle tissue is exemplified by the loss in muscle mass that occurs under periods of disuse, the ability to regenerate this mass when normal activity is resumed, and the ability of muscle to grow as functional demand increases. ${ }^{29}$ Skeletal muscle cells can actively participate in local immune reactions. ${ }^{30}$ Myofibers and myoblasts express cytokine receptors, and are capable of producing cytokines influencing the course of inflammation. $^{13}, 23,31,32$ A growing body of evidence suggests that, in addition to immune/inflammatory responses, cytokines also influence normal physiologic functions of skeletal muscle cells including anabolic and catabolic processes and apoptosis. ${ }^{11,13}$ Therefore, cytokines play an important role in muscle homeostasis, disease pathogenesis, and injury responses. Despite the importance of cytokines as regulators of skeletal muscle function, there are many gaps in our understanding of how cytokines affect muscle function under normal, injured, and pathogenic circumstances. ${ }^{11}$ Understanding the mechanisms involved in modulating skeletal muscle phenotypes is necessary to improve the treatment of muscular diseases, and to understand the changes that muscle tissue undergoes in response to traumatic injury.

Inflammatory responses can be induced by a wide spectrum of injury severities in muscle tissue ranging from overexertion to traumatic injury. Adult skeletal muscle has a remarkable and unique capacity for regeneration following injury. ${ }^{25}$ Traumatic muscle injury initiates an extensive and rapid repair process consisting of degeneration, inflammation, regeneration, and fibrosis that takes place at the tissue, cellular, and molecular levels. ${ }^{24,25,33}$ Muscle healing is a delicate balance between regeneration of normal muscle and formation of scar tissue, where the inflammatory response can be both beneficial and damaging. If fibrotic tissue develops during the healing process, the muscle's function may become limited. ${ }^{24}$ Only a small number of immune cells reside within intact skeletal muscle. ${ }^{33}$ Muscle injury causes the release of normally 
cytosolic molecules which can initiate the release of cytokines and rapid invasion by inflammatory cell populations that can last for days to weeks while repair, regeneration, and growth occur. $^{11,31,33}$

Muscle growth and injury-induced regeneration in muscle is dependent on postnatal muscle stem cells called satellite cells. ${ }^{25,}{ }^{34}$ Satellite cells reside in an isolated microenvironment between the thin sheet of membrane lining the surface of muscle (basement membrane) and the cell membranes (sarcolemma) of individual myofibers. ${ }^{35}$ Following tissue damage the normally quiescent satellite cells become active and migrate, proliferate, and differentiate to aid in repair and regeneration. ${ }^{24,33}$ Satellite cells differentiate to form mononucleated myoblasts which then fuse together to form multinucleated myofibers. ${ }^{33}$ In addition, satellite cells can also differentiate to form bone or fat cells. ${ }^{33}$ The regeneration process is heavily reliant on the dynamic interplay between satellite cells and the structural and biochemical cues emanating from their local molecular environments. ${ }^{36,} 37$ Immune cells and soluble factors such as cytokines recruited to injured muscle tissue constitute a transient local environment for satellite cells. ${ }^{38}$ Satellite cells and immune cells can attract each other through cytokine secretion. Satellite cells have been demonstrated to secret cytokines such as IL-6 and TNF- $\alpha$ to facilitate immune cell infiltration and function. ${ }^{39}$ Immune cells in turn secrete factors that help satellite cells escape from their sequestered compartments and promote satellite cell proliferation. ${ }^{40}$ Throughout regeneration, the collaborative behaviors of satellite cells and other cells are heavily influenced by changes to their local molecular environments. ${ }^{25,31,33}$ It is clear that these spatial differences in the molecular environment of the various cells involved in the wound healing process is a key aspect of wound healing process, warranting further investigation until a complete spatial 
mechanistic understanding of the complex molecular mechanisms that underlie skeletal muscle healing is achieved.

As stated before, the response of skeletal muscle to traumatic injury is a complex, highly coordinated process that relies on precise spatial and temporal regulation. Cytokine responses have been extensively studied as important mediators of the response to trauma, ${ }^{20,41}$ and MAPKs are recognized as being key to stress responses influenced by cytokine signals. ${ }^{5}$ The understanding that inflammatory cytokine responses can be both damaging and beneficial has led to the use of both cytokine suppressing agents and cytokines themselves as pharmacological treatments even without a thorough mechanistic understanding of cytokine signaling. ${ }^{12}$ The immediate challenge is to advance the current understanding of the networked relationships between the heterogeneous cell populations and molecules that coordinate the injury response in complex and dynamic in vivo environments. In this chapter, the network analysis approach developed in this body of work was used to integrate experimental data obtained from injured muscle tissue, and map the complex network responses involved in the tissue response to trauma.

\subsection{Experimental Procedures}

\subsubsection{Animals}

Adult male Sprague-Dawley rats were housed individually with a 12:12 light/dark cycle with ad libitum access to standard rat chow and water. Four time points were studied with 3 replicates each $(\mathrm{N}=3)$ for a total of 12 rats for the study. All procedures were performed under the guidelines approved by the West Virginia University Animal Care and Use Committee. 


\subsubsection{Femur Fracture}

After adequate anesthesia all animals were subjected to a standardized femur fracture on one leg using a custom designed tool in which a weight is dropped in a consistent fashion onto the mid-shaft of the rat's thigh. ${ }^{42}$ This tool delivers a calculated force of 104.80 Newtons, generating a reproducible femur fracture and associated soft tissue injury. ${ }^{42}$ Buprenorphine SR was pre-operatively administered subcutaneously as an analgesic providing 72 hour pain relief. Rats were anesthetized intraperitoneally with Ketamine $(80-90 \mathrm{mg} / \mathrm{kg})$ and Xylazine (10-15 $\mathrm{mg} / \mathrm{kg}$ ). This combination of analgesic and anesthetics has previously been identified as the best combination for avoiding significant modulation of cytokine responses in a rat model. ${ }^{43}$ Additionally, analgesics and anesthetics were injected into the scruff on the back of the rat's neck, thus there was an equal distance between the injection and both legs. An incision was made to visualize the fracture. A hole was drilled into the proximal femur to allow a 0.045 inch Kirschner wire (K-wire) to be inserted down the intramedullary canal to fix the fracture. The incision was closed starting with the fascia and then using a stainless steel suture on the skin. Rats were subcutaneously administered Yohimbine $(2 \mathrm{mg} / \mathrm{kg}$ ) post-operatively to reverse the Xylazine and were closely observed during recovery for signs of distress.

\subsubsection{Sample Preparation}

Three rats were sacrificed at each of 4 time points $(0,6,24$, and $168 \mathrm{~h}$ post-fracture). Rats were anesthetized intraperitoneally with Ketamine $(80-90 \mathrm{mg} / \mathrm{kg})$ and Xylazine (10-15 $\mathrm{mg} / \mathrm{kg}$ ). One cc of Euthasol was then administered via intracardiac puncture. Muscle tissue was then harvested from the following 3 locations: at the site of the fracture, $1.0 \pm 0.2 \mathrm{~cm}$ away from the site of fracture, and from the leg opposite to the fractured leg. Figure 4-1 illustrates the sampling locations. Samples were immediately rinsed with ice cold phosphate buffered saline 
(PBS), snap frozen, and stored at $-80^{\circ} \mathrm{C}$. Protein extraction was achieved using methods adapted from those of Hulse et al. ${ }^{44}$ Samples were ground cryogenically and then lyophilized. For analyses, 2-3 mg of lyophilized tissue sample was thawed for $10 \mathrm{~min}$ at $4^{\circ} \mathrm{C}$ in cell lysis buffer (Bio-Rad, Hercules, CA) containing $20 \mathrm{mM}$ phenylmethylsulfonyl fluoride (Sigma, St. Louis, MO). Thawed samples were then vortexed for $1-3 \mathrm{sec}$, and homogenized with 3 rapid pulses using an ultrasonic dismembrator. Samples were vortexed again for 1-3 sec and centrifuged at $5,000 \mathrm{x} \mathrm{g}$ for $5 \mathrm{~min}$ at $4^{\circ} \mathrm{C}$. The supernatant was collected and total protein concentration was determined using the RCDC protein assay (Bio-Rad, Hercules, CA) according to the manufacturer's instructions. Absorbance values were determined using an Infinite M1000 plate reader (Tecan, Raleigh, NC).

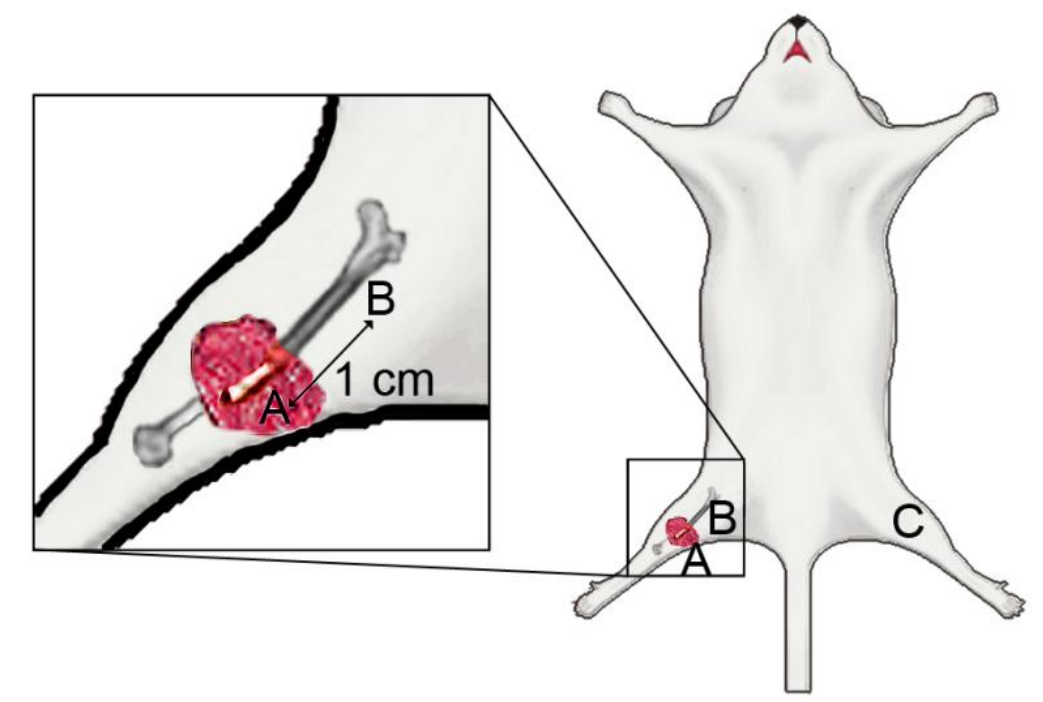

Figure 4-3. Sampling locations following femur fracture. Muscle and vessel samples were obtained from three different locations: (A) directly at the fracture site (B) $1 \mathrm{~cm}$ away from the fracture site (C) from the opposite un-injured leg.

\subsubsection{Analyte Measurement}

Sample homogenates were diluted to a total protein concentration of $900 \mu \mathrm{g} / \mathrm{ml}$ with sample diluent (Bio-Rad, Hercules, CA), and assayed for the cytokine targets, GM-CSF, IL-1a, 
IL-1ß, IL-2, IL-6, MIP-1a, and TNF-a, using the Bio-Plex Pro multiplexed magnetic bead-based immunoassay reagent kit containing magnetic antibody coated beads with the Bio-Plex 200 suspension array system and Pro II Wash Station (Bio-Rad, Hercules, CA) according to the manufacturer's instructions. The cytokine targets VCAM and ICAM were assayed using the Procarta multiplexed magnetic bead-based immunoassay reagent kit with the Bio-Plex 200 suspension array system and Pro II Wash Station (Bio-Rad, Hercules, CA) according to the manufacturer's instructions. Phosphoproteins were assayed using the Bio-plex phosphoprotein kit containing polystyrene, non-magnetic antibody coated beads specific for the following targets phosphorylated at the indicated amino acid residues: Akt (Ser472), c-Jun (Ser63), CREB (Ser133), ERK1/2 (Thr202/Tyr204, Thr185/Tyr187), GSK-3a/ß (Ser21/Ser9), HSP27(Ser78), JNK (Thr183/Tyr185), MEK1 (Ser217/Ser221), NF-kB (Ser536) p38 (Thr180/Tyr182), p70S6 (Thr421/Ser424), p90RSK (Thr359/Ser363), STAT2 (Tyr689), STAT3 (Tyr705). The relative abundance of total protein was determined using a Bio-plex kit containing polystyrene, nonmagnetic antibody coated beads specific for Akt, c-Jun, CREB, ERK1/2, HSP27, JNK, MEK1, p38, and p90RSK.

\subsubsection{Statistical Analysis.}

Data were analyzed using Prism 5 (GraphPad, San Diego, CA). Protein abundances were compared based on the blank subtracted intensity of the relative fluorescence intensity (RFI) measured for each antibody-coated bead. Two-way analysis of variance (ANOVA) with Bonferroni's post test was used to determine significant differences between each sampling location and between time points. All fluorescence measurements were performed in duplicate. Data are expressed as the mean \pm standard error of the mean (SEM). 


\subsubsection{Network Analysis}

Radiality values were calculated using Cytoscape and the CentiScape plugin. To account for the variable sensitivity in the detection of the different targets, relative fluorescent intensity signals were normalized for each individual protein target based on the minimum and maximum values observed for the particular target across all samples to range from 0 to $100 \%$ using Prism 5. The Euclidean distances between these normalized values were used as distance values for the radiality calculations performed using Cytoscape as described in chapter 2.

\subsection{Results}

\subsubsection{Spatial Relative Fluorescence Intensity Comparisons}

The spatial cytokine response data for IL-6, IL-1 $\alpha$, IL-1ß, IL-2, GM-CSF, TNF- $\alpha$, and MIP-1 $\alpha$ have been published in the journal Cytokine. ${ }^{45}$

\subsubsection{Cytokine Spatial Response Comparisons}

Figure 4-2 shows the relative fluorescence intensity (RFI) values obtained for the assayed cytokines at each sampling location at each of the four time points. Comparisons between the RFI for each individual analyte at different locations were performed using 2-way ANOVA with Bonferroni's post test, and significant differences are indicated on Figure 4-2. Statistically significant $(p<0.05)$ differences in RFI values when comparing the different sampling locations were observed in samples taken immediately following the femur fracture $(0 \mathrm{~h}$ time point $)$ for IL-2, TNF-a, MIP-1a, and ICAM. For samples taken $6 \mathrm{~h}$ following the injury statistically significant differences in RFI values at the different physical locations were observed for IL-1ß and IL-6. For samples taken $24 \mathrm{~h}$ after the femur fracture, the only significant difference 
between the sampling locations was in IL- $1 \beta$ associated RFI. The only statistically significant difference in RFI values for samples taken $168 \mathrm{~h}$ after the femur fracture was in VCAM levels. 


\section{Cytokines}
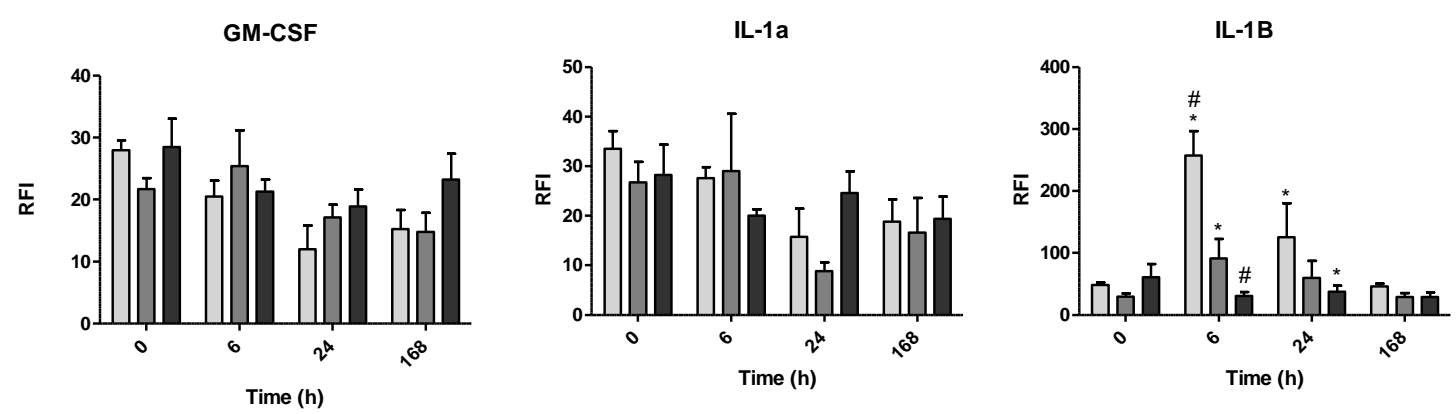

IL-2
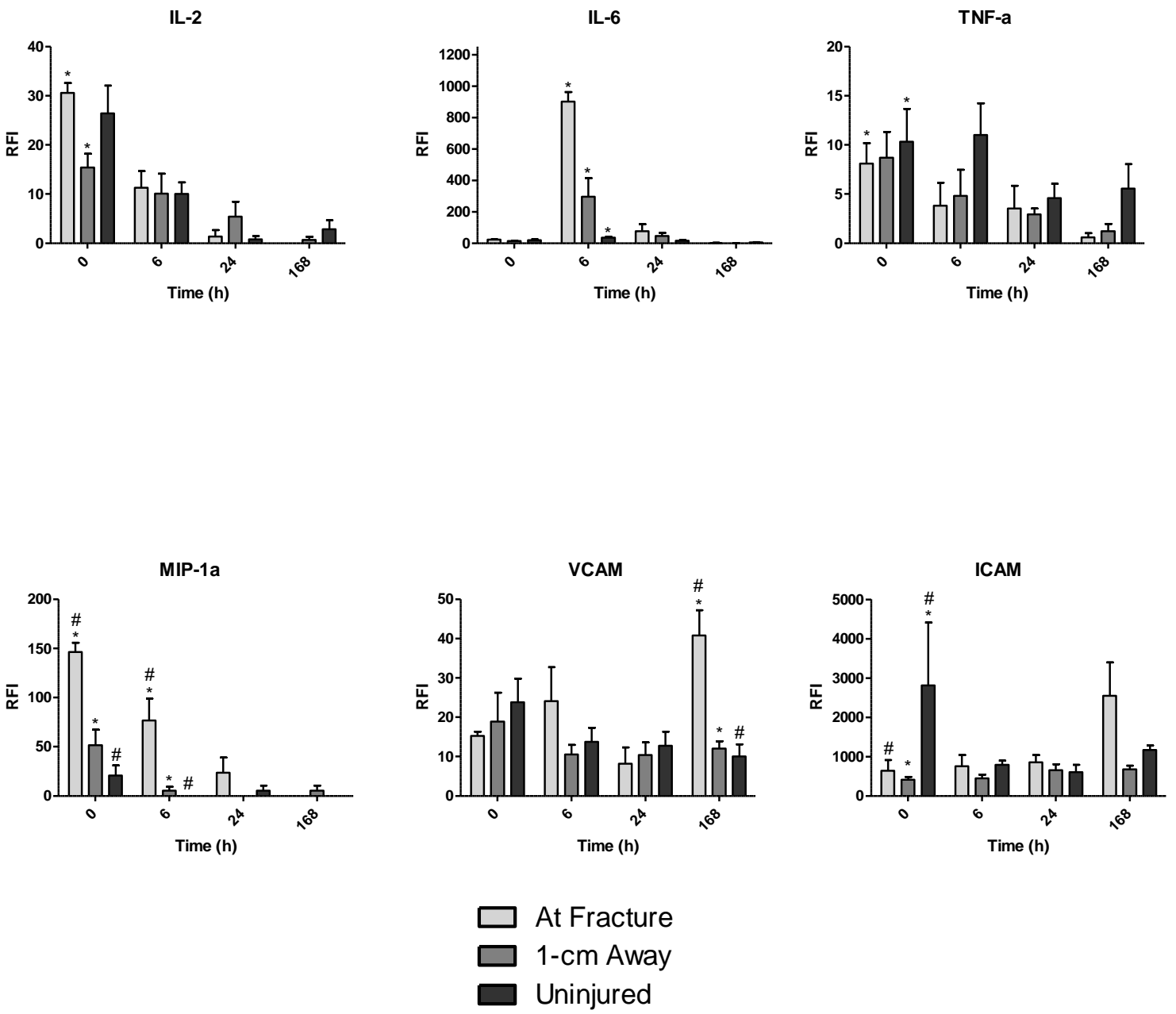

Figure 4-2. Cytokine associated RFI following femur fracture. 


\subsubsection{Phosphoprotein Spatial Response Comparisons}

Figures 4-3 and 4-4 show RFI values obtained for the assayed phosphoproteins at each sampling location and at each time point. Statistically significant differences $(p<0.05)$ in RFI values were observed across the 3 locations sampled for many of the assayed proteins, and these significant differences are indicated in Figures 4-3 and 4-4. For samples collected immediately following the fracture $(0 \mathrm{~h}$ time point $)$, RFI was significantly higher in the uninjured tissue samples than in tissue samples taken directly from the fracture site and tissue taken $1 \mathrm{~cm}$ away from the fracture site for phosphorylated Akt, c-Jun, ERK1/2, GSK-3 $\alpha / \beta$, HSP27, and p70S6. Phosphorylated CREB associated RFI values were also significantly different immediately following femur fracture, but only when comparing samples from the uninjured leg to samples taken from $1 \mathrm{~cm}$ away from the fracture. Significant differences in RFI values between sampling locations were observed at the $6 \mathrm{~h}$ time point for phosphorylated Akt, GSK-3 $\alpha / \beta$, and MEK1. Significant differences in RFI values between sampling locations were observed at the $24 \mathrm{~h}$ time point for phosphorylated p38 and GSK-3 $\alpha / \beta$. Significant differences in RFI values between sampling locations were observed at the $168 \mathrm{~h}$ time point for phosphorylated p38, p70S6, and MEK1. No statistically significant differences in RFI for phosphorylated JNK, NFkB, p90RSK, or STAT2 were observed when comparing any of the sampling locations at any time point. 


\section{Phosphoproteins}
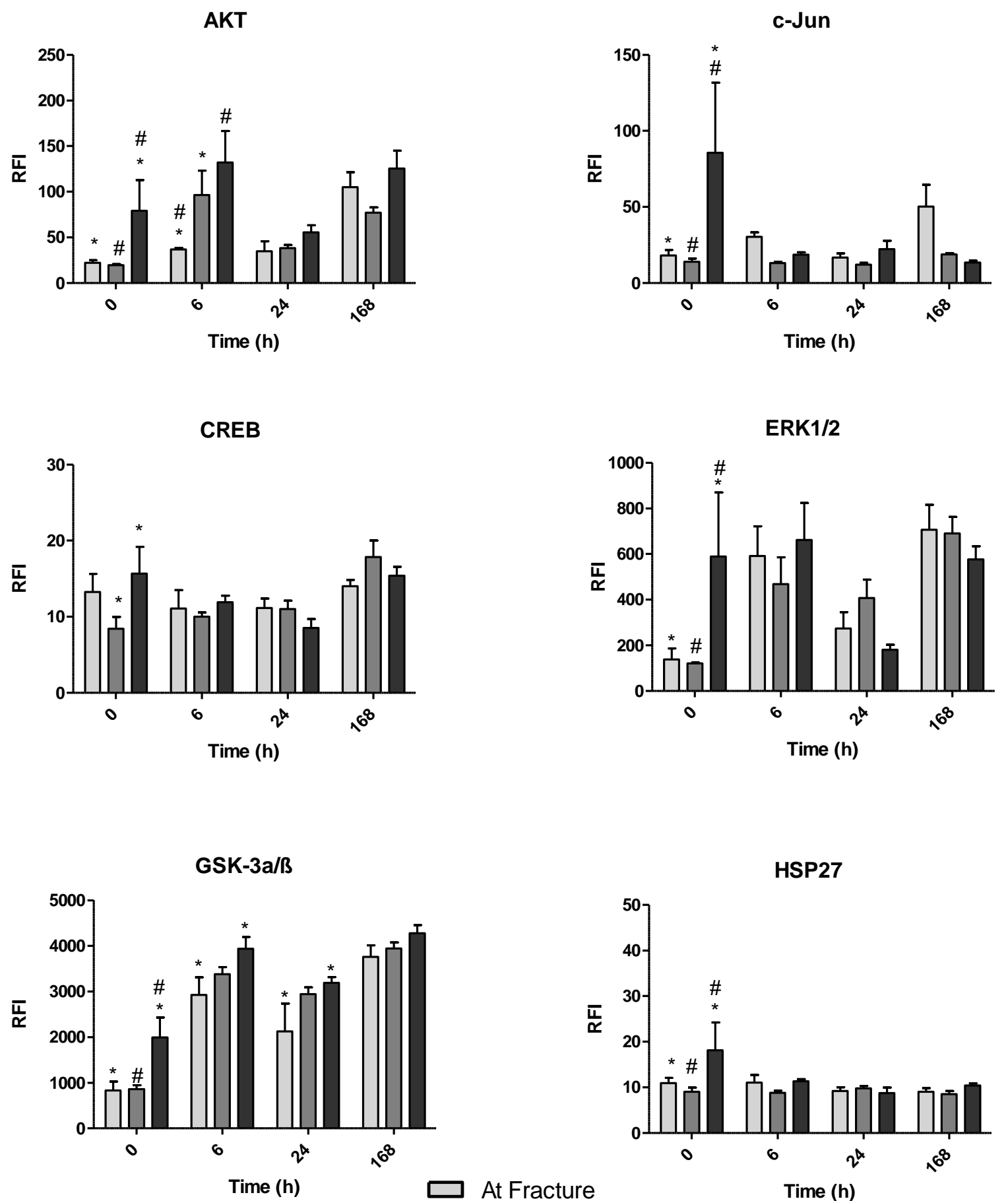

At Fracture

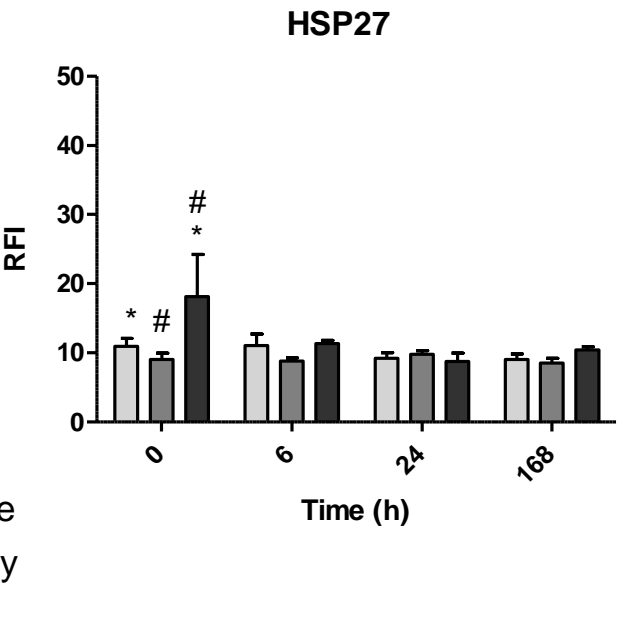

Figure 4-3. Phosphoprotein associated RFI following femur fracture. 

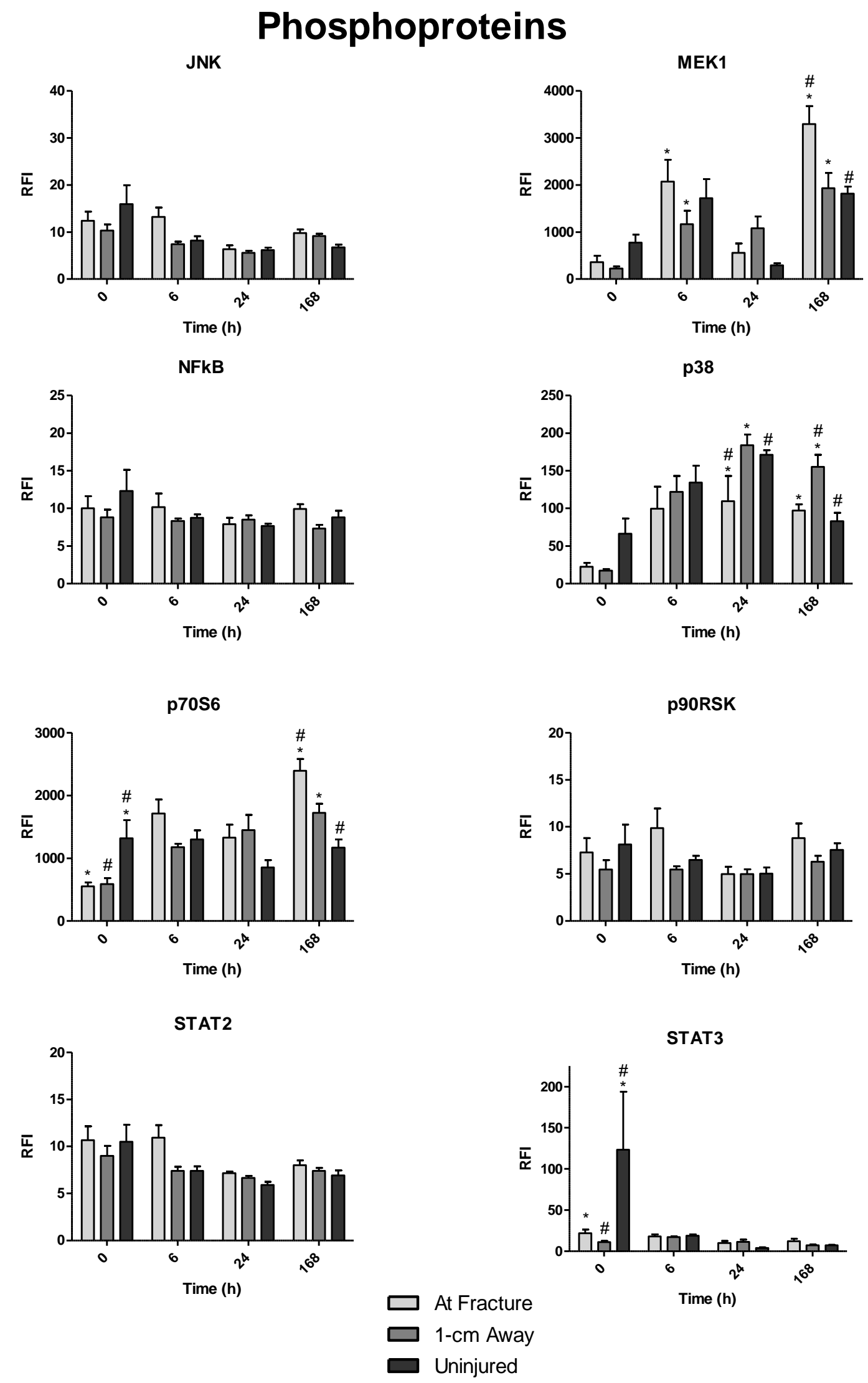

Figure 4-4. Phosphoprotein associated RFI following femur fracture. 


\subsubsection{Total Protein Spatial Response Comparisons}

Figure 4-5 shows total protein associated RFI values assayed at each sampling location and at each time point. Statistically significant $(p<0.05)$ differences in total protein RFI values were observed when comparing the different sampling locations for all of the studied time points, and are depicted in Figure 4-5. In samples taken immediately following the femur fraction, significant differences in Akt and ERK1/2 total protein associated RFI values were found when comparing the different sampling locations. For samples taken $6 \mathrm{~h}$ following the fracture, p38 was the only protein with significantly different RFI values between sampling locations. Significant spatial differences for total MEK1 and p38 RFI values were observed in samples taken $24 \mathrm{~h}$ after the femur fracture. The most significant differences between different sampling locations for total protein associated RFI values were observed in samples taken $168 \mathrm{~h}$ after the femur fracture. Akt, c-Jun, ERK1/2, JNK, and p38 all had significant spatial differences in RFI values at this time point. 


\section{Total Protein}
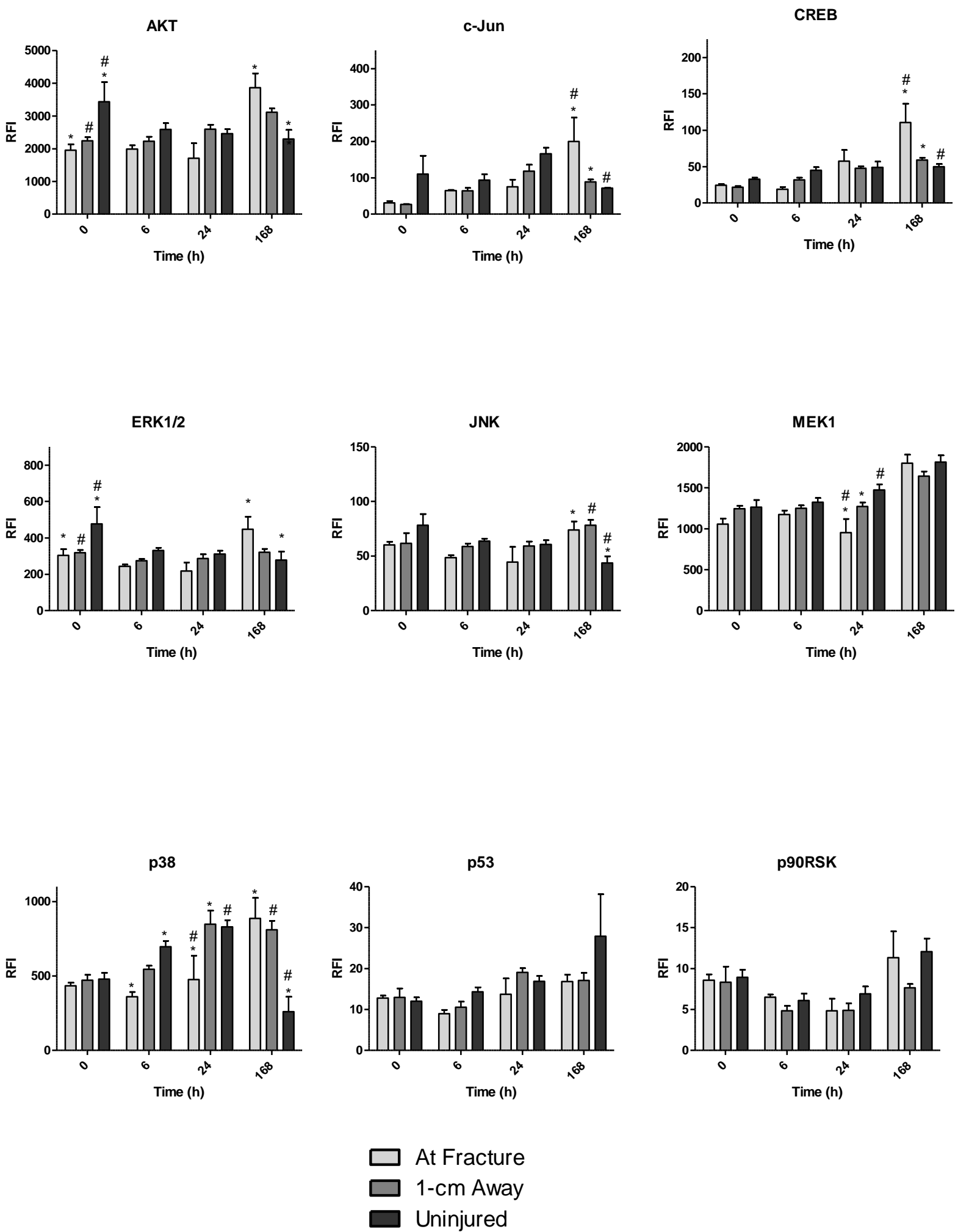

Figure 4-5. Total protein associated RFI following femur fracture. 


\subsubsection{Network Analysis}

Radiality parameters were calculated for each of the 3 rats separately, and a mean radiality values was calculated for all 3 rats. Radiality values were calculated separately for each individual time point and location. Graphs depicting changes in mean radiality values over time were constructed for each location. Two additional analyses were performed, the first incorporated protein responses at all 3 locations into the radiality calculation to give one radiality value for the entire rat at each time point. This analysis is referred to as the across locations analysis. The second analysis incorporated protein responses from all 4 time points into the radiality calculation to give one radiality value for each location. This analysis is referred to as the across time analysis. For all analyses, mean radiality values for each individual protein target were compared to the mean radiality value obtained for all proteins targets \pm the standard deviation (SD). Two-way ANOVA with Bonferroni's post test was used to determine protein targets with significantly different $(\mathrm{p}<0.05)$ radiality values at the same time points and locations.

\subsubsection{Radiality Comparisons at Fracture}

Figure 4-6 depicts protein radiality values at the fracture site. For the network analysis performed based on the response at the fracture site, immediately following the injury, IL-2's radiality was significantly lower than 14 other proteins. IL-2 was the only protein with a radiality value that was significantly different from other proteins at this location and time point. Immediately following femur fracture IL-2, GM-CSF, TNF- $\alpha$, and phosphorylated STAT2 all had radiality values less than the mean radiality value calculated for all proteins minus the SD.

For the network analysis performed based on the response at the fracture site $6 \mathrm{~h}$ following the injury, IL-6's radiality was significantly lower than 6 other proteins. IL-6 was the 
only protein with a radiality value that was significantly different from other proteins at this location and time point. IL-6, VCAM and IL-1ß all had radiality values less than the mean radiality value calculated for all proteins minus the SD.

There were no statistical differences in protein radiality values for the $24 \mathrm{~h}$ time point at the fracture site. Phosphorylated p38, phosphorylated GSK-3 $\alpha / B$, and total JNK all had radiality values less than the mean radiality value calculated for all protein targets minus the SD. There were no statistical differences in protein radiality values for the $168 \mathrm{~h}$ time point at the fracture site. Phosphorylated GSK-3 $\alpha / 3$, VCAM, total MEK1, IL-6, phosphorylated p70S6, and phosphorylated STAT3 all had radiality values less than the mean radiality value calculated for all protein targets minus the SD.

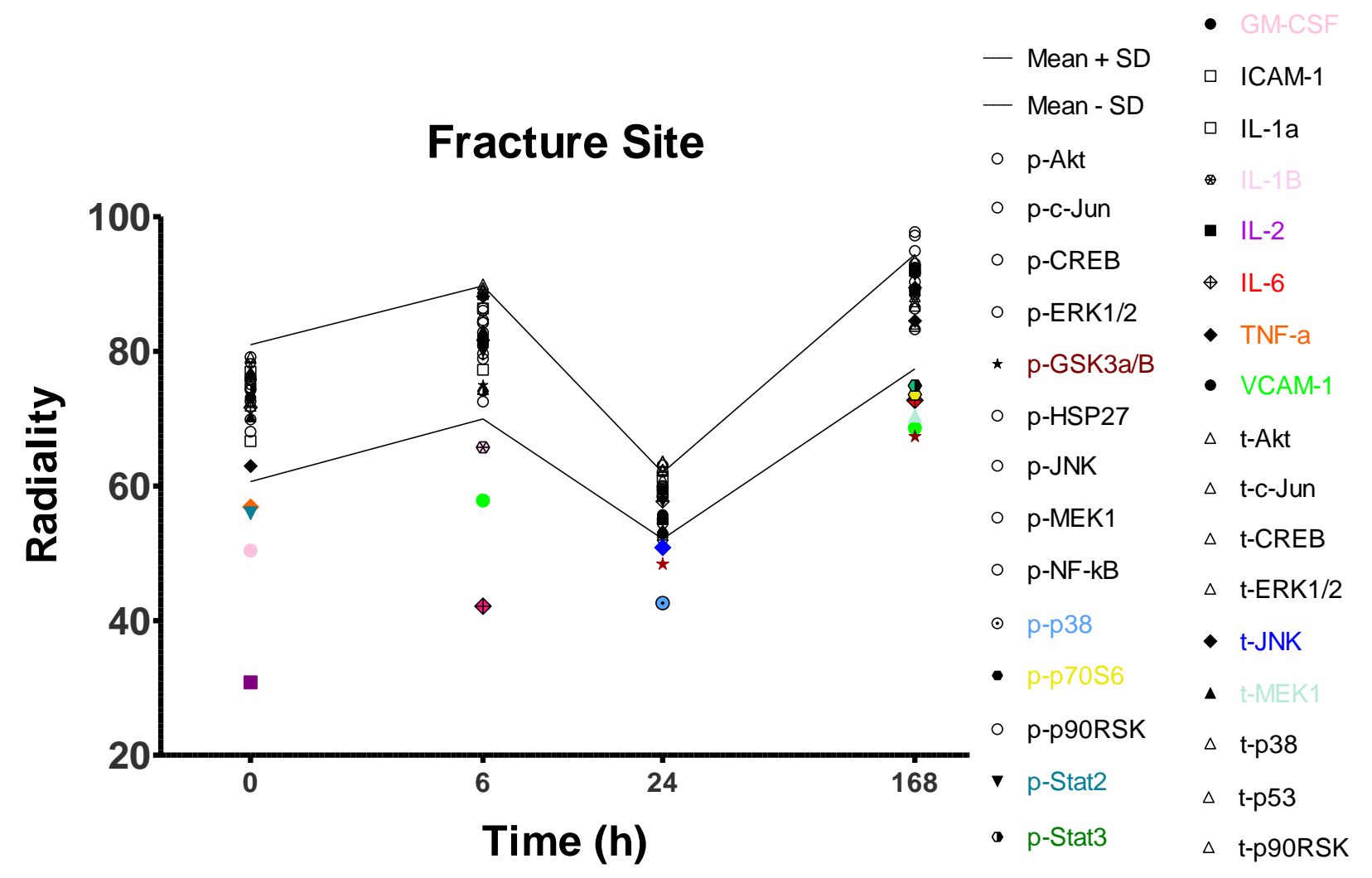

Figure 4-6. Protein radiality values calculated based on protein responses measured in muscle sampled at the fracture site. Proteins with radiality less than the mean observed radiality minus the standard deviation are shown in color. Phosphorylated proteins are indicated with " $p$ ". Total proteins are indicated with " $\mathrm{t}$ ". 


\subsubsection{Radiality Comparisons $1 \mathrm{~cm}$ Away from Injury}

Figure 4-7 depicts protein radiality values $1 \mathrm{~cm}$ away from the fracture. There were no significant differences in protein radiality values at the $0 \mathrm{~h}$ time point for the $1 \mathrm{~cm}$ away network analysis. VCAM, total p38, IL-2, total JNK, and GM-CSF all had radiality values less than the mean radiality value calculated for all protein targets minus the $\mathrm{SD}$ for the $0 \mathrm{~h}$ time point.

GSK-3 $\alpha / \beta$ had significantly lower radiality values in comparison to 9 proteins at the $6 \mathrm{~h}$ time point. Phosphorylated GSK-3 $\alpha / \beta$, GM-CSF, and phosphorylated p38 all had radiality values less than the mean radiality value calculated for all protein targets minus the SD for the 6 $\mathrm{h}$ time point.

Phosphorylated p38 had a significantly lower radiality value in comparison to 27 other proteins at the $24 \mathrm{~h}$ time point. Total p53 radiality was significantly lower in comparison to 2 proteins at the $24 \mathrm{~h}$ time point. Phosphorylated GSK-3 $\alpha / \beta$ had a significantly lower radiality value than 1 protein at the $24 \mathrm{~h}$ time point. Phosphorylated p38, phosphorylated GSK-3 $\alpha / \beta$, and total p53 were the only protein targets with radiality values less than the mean radiality value calculated for all protein targets minus the SD for the $24 \mathrm{~h}$ time point.

GSK-3 $\alpha / \beta$ had a significantly lower radiality value in comparison to 21 proteins at the $168 \mathrm{~h}$ time point. Phosphorylated GSK-3 $\alpha / \beta$, total MEK1, total JNK, and phosphorylated p38 all had radiality values less than the mean radiality value calculated for all protein targets minus the SD for the $168 \mathrm{~h}$ time point. 


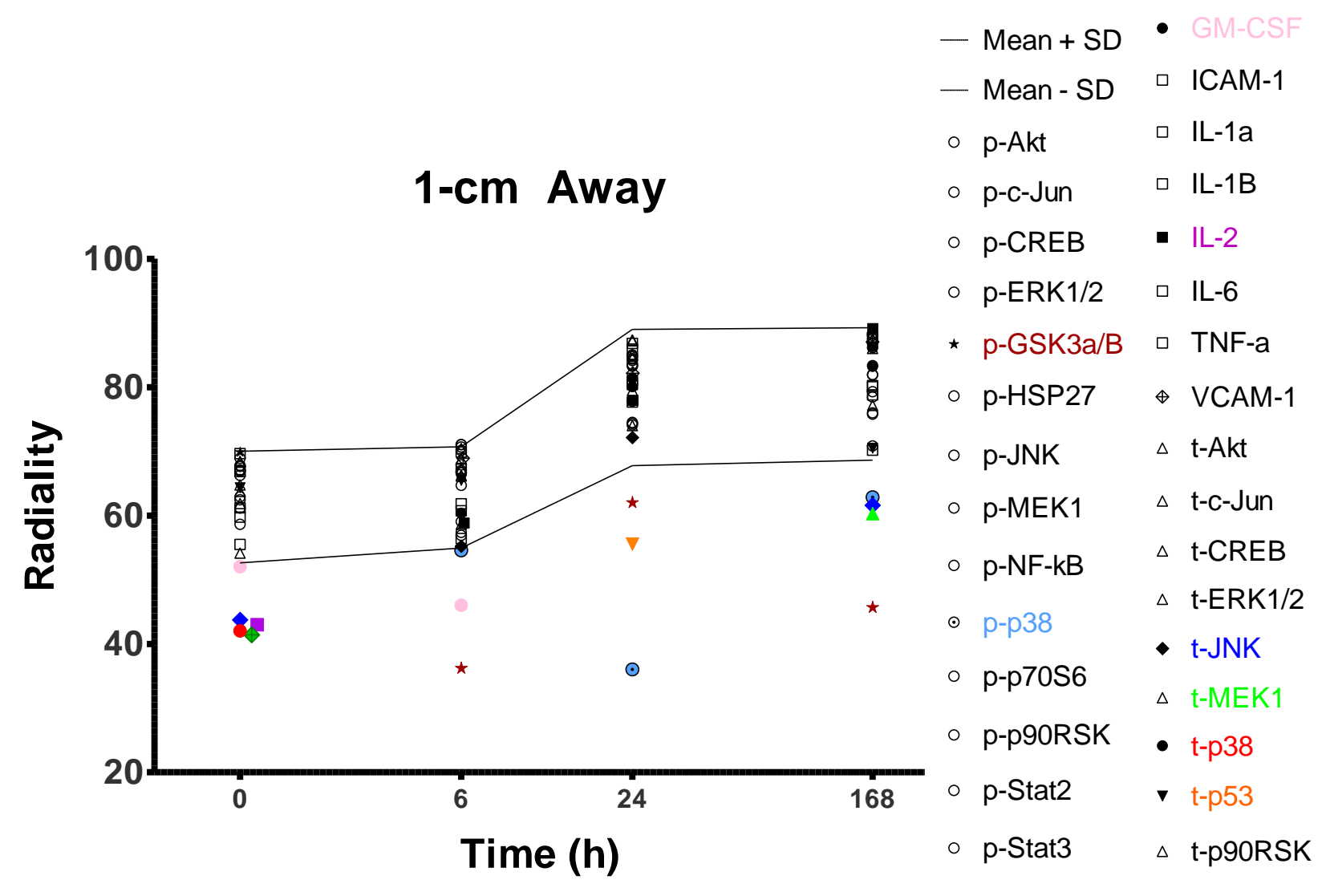

Figure 4-7. Protein radiality values calculated based on protein responses measured in muscle sampled $1 \mathrm{~cm}$ away from the fracture site. Proteins with radiality less than the mean observed radiality minus the standard deviation are shown in color. Phosphorylated proteins are indicated with " $p$ ". Total proteins are indicated with " $\mathrm{t}$ ".

\subsubsection{Radiality Comparisons Uninjured Leg}

Figure 4-8 depicts radiality values for the uninjured leg. For the network analysis performed based on the response in the uninjured leg, many of the radiality values for the assayed proteins were significantly different from each other at the 0 and $6 \mathrm{~h}$ time points. Most of these proteins with significantly different radiality values were cytokines.

At the $0 \mathrm{~h}$ time point phosphorylated p70S6, phosphorylated p38, phosphorylated MEK11, phosphorylated HSP27, and phosphorylated NFkB radiality values were significantly different than many of the other proteins targets. ICAM, TNF- $\alpha$, VCAM, total p90RSK, and 
total CREB all had radiality values less than the mean radiality value calculated for all protein targets minus the SD for the $0 \mathrm{~h}$ time point.

Phosphorylated CREB, IL- 1ß, ICAM, phosphorylated Akt, phosphorylated ERK1/2, and GM-CSF all had radiality values less than the mean radiality value calculated for all protein targets minus the SD for the $6 \mathrm{~h}$ time point.

Phosphorylated CREB, total MEK1, total JNK, phosphorylated p38, and IL-6 all had radiality values less than the mean radiality value calculated for all protein targets minus the SD for the $24 \mathrm{~h}$ time point.

Phosphorylated STAT3, TNF- $\alpha$, total JNK, phosphorylated STAT2, and phosphorylated MEK1 all had radiality values less than the mean radiality value calculated for all protein targets minus the SD for the $168 \mathrm{~h}$ time point. 

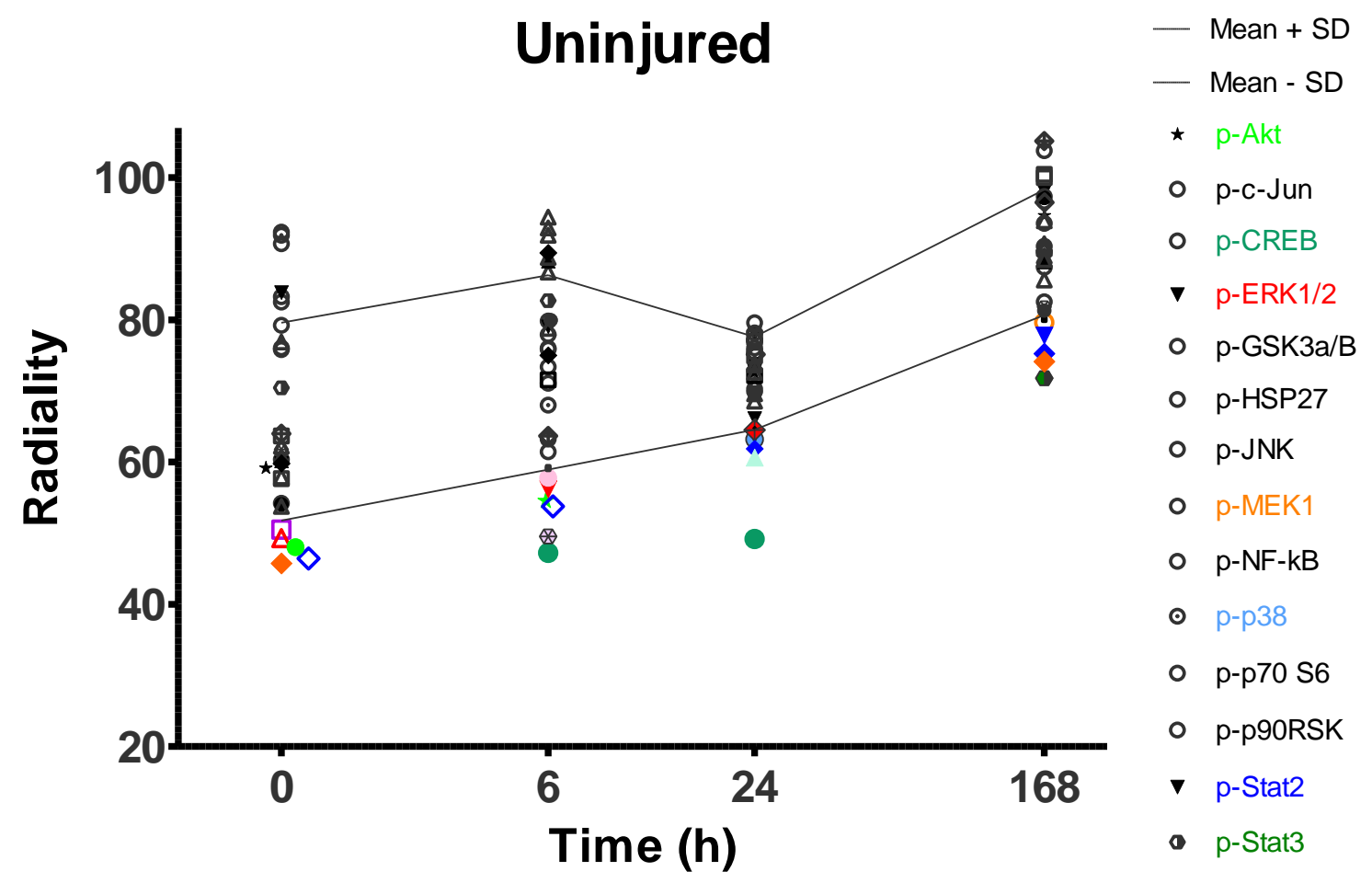

$\diamond$ ICAM-1

c IL-1a

IL-2

$\bullet$ IL-6

- TNF-alpha

- VCAM-1

$\Delta$ t-Akt

$\Delta \quad$ t-c-Jun

口 t-CREB

$\Delta \quad \mathrm{t}-\mathrm{ERK} 1 / 2$

- t-JNK

$\Delta$

$\Delta \quad \mathrm{t}-\mathrm{p} 38$

$\Delta \quad \mathrm{t}-\mathrm{p} 53$

$\Delta$ t-p90RSK

Figure 4-8. Protein radiality values calculated based on protein responses measured in muscle sampled from the uninjured leg. Proteins with radiality less than the mean observed radiality minus the standard deviation are shown in color. Phosphorylated proteins are indicated with " $p$ ". Total proteins are indicated with " $\mathrm{t}$ ".

\subsubsection{Across Locations Radiality Comparisons}

To depict the global network response to the injury, radiality values were also calculated across the sampling locations by including the protein responses from all 3 locations in the Euclidean distance calculation. This results in 1 radiality value for each protein at each time point. Figure 4-9 shows the radiality values calculated using normalized RFI values from all three locations. Statistical analysis was performed to determine proteins with significantly different radiality values at each time point.

At the $0 \mathrm{~h}$ time point, IL-2 was the only protein to have a significantly different radiality value in comparison to the other proteins. At this time point, IL-2's radiality was significantly lower in comparison to 23 other protein targets. IL-2, TNF- $\alpha$, GM-CSF, and total JNK all had 
radiality values less than the mean radiality value calculated for all protein targets minus the SD for the $0 \mathrm{~h}$ time point.

At the $6 \mathrm{~h}$ time point IL- 6 radiality was significantly lower than 13 of the other proteins, and phosphorylated GSK-3 $\alpha / \beta$ was significantly lower than 17 of the other proteins. Phosphorylated GSK-3 $\alpha / \beta$ and IL-6 were the only proteins with radiality values less than the mean radiality value calculated for all protein targets minus the SD for the $6 \mathrm{~h}$ time point.

At the $24 \mathrm{~h}$ time point, phosphorylated p38's radiality was significantly lower than 27 of the other proteins, total p38 was significantly lower than 7 of the other proteins, and phosphorylated GSK-3 $\alpha / \beta$ radiality was significantly lower than 3 of the assayed proteins. Phosphorylated $\mathrm{p} 38$, total $\mathrm{p} 38$, and phosphorylated GSK-3 $\alpha / \beta$ all had radiality values less than the mean radiality value calculated for all protein targets minus the SD for the $24 \mathrm{~h}$ time point.

At the $168 \mathrm{~h}$ time point, phosphorylated GSK-3 $\alpha / \beta$ was significantly lower than 29 other proteins, and total MEK1 radiality was significantly lower than 13 other proteins. Phosphorylated GSK-3 $\alpha / \beta$ and total MEK1 were the only proteins with radiality values less than the mean radiality value calculated for all protein targets minus the SD for the $168 \mathrm{~h}$ time point. 


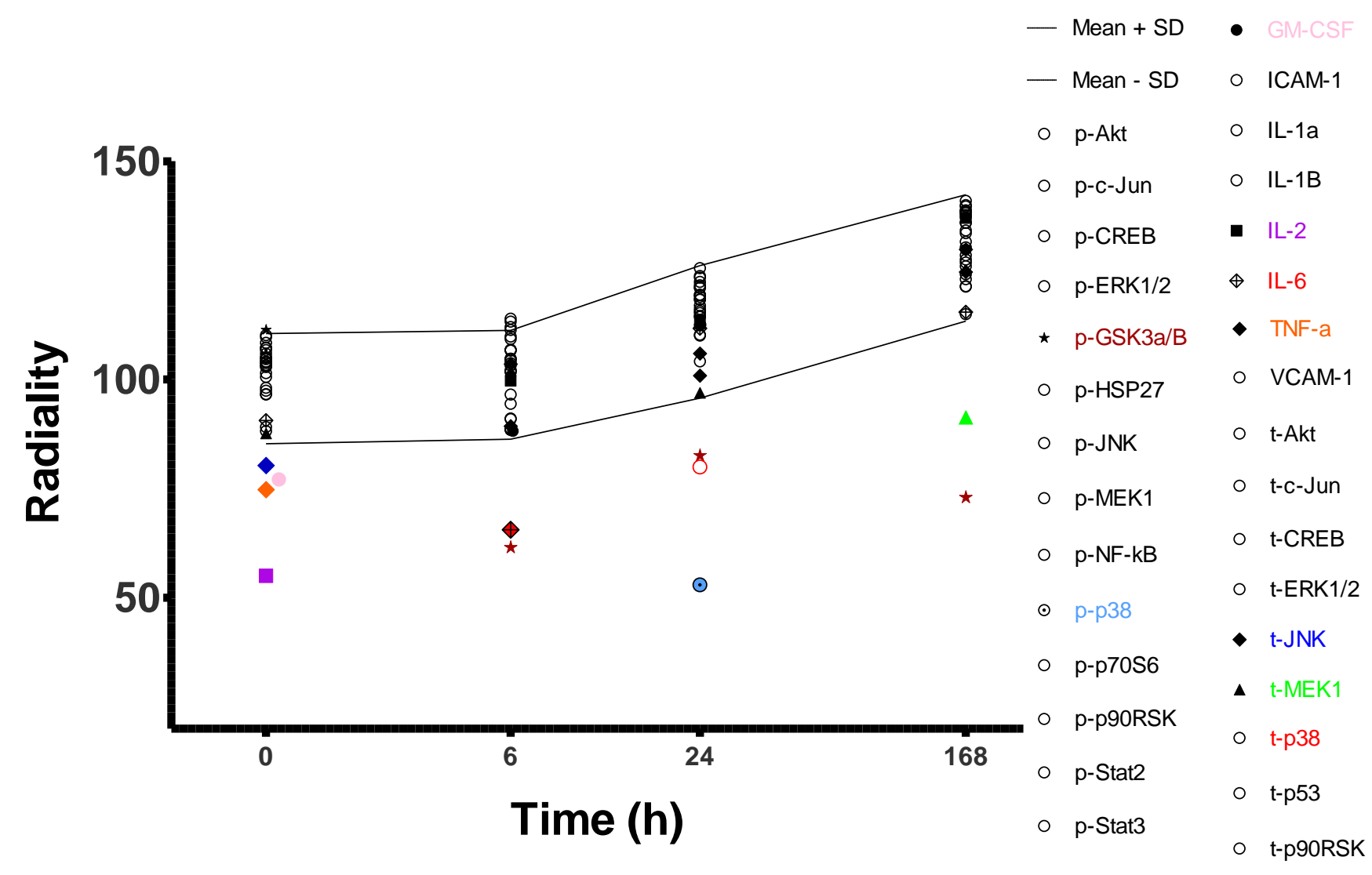

Figure 4-9. Across locations radiality. Radiality values were calculated based on protein responses at all 3 sampling locations. Proteins with radiality less than the mean observed radiality minus the standard deviation are shown in color.

\subsubsection{Across Time Radiality Comparisons}

Radiality values were also calculated across the sampling time points by including the protein responses from all 4 time points in the Euclidean distance calculation. This results in 1 radiality value for each sampling location. Figure 4-10 shows the radiality values calculated using normalized RFI values from all 4 time points. Statistical analysis was performed to determine proteins with significantly different radiality values at each location. At the fracture site IL-2, IL-6, and VCAM radiality values were significantly $(\mathrm{p}<0.05, \mathrm{p}<0.001, \mathrm{p}<0.05$, respectively) different from the mean. At the $1 \mathrm{~cm}$ away location, phosphorylated GSK- $3 \alpha / \beta$, phosphorylated p38, and total JNK radiality values were significantly different $(\mathrm{p}<0.001, \mathrm{p}<$ 
$0.001, \mathrm{p}<0.05$, respectively) from the mean. For the uninjured leg phosphorylated GSK-3 $\alpha / \beta$, phosphorylated $\mathrm{p} 38$, and total MEK radiality values were significantly different $(\mathrm{p}<0.001, \mathrm{p}<$ $0.05, \mathrm{p}<0.001$, respectively) from the mean.

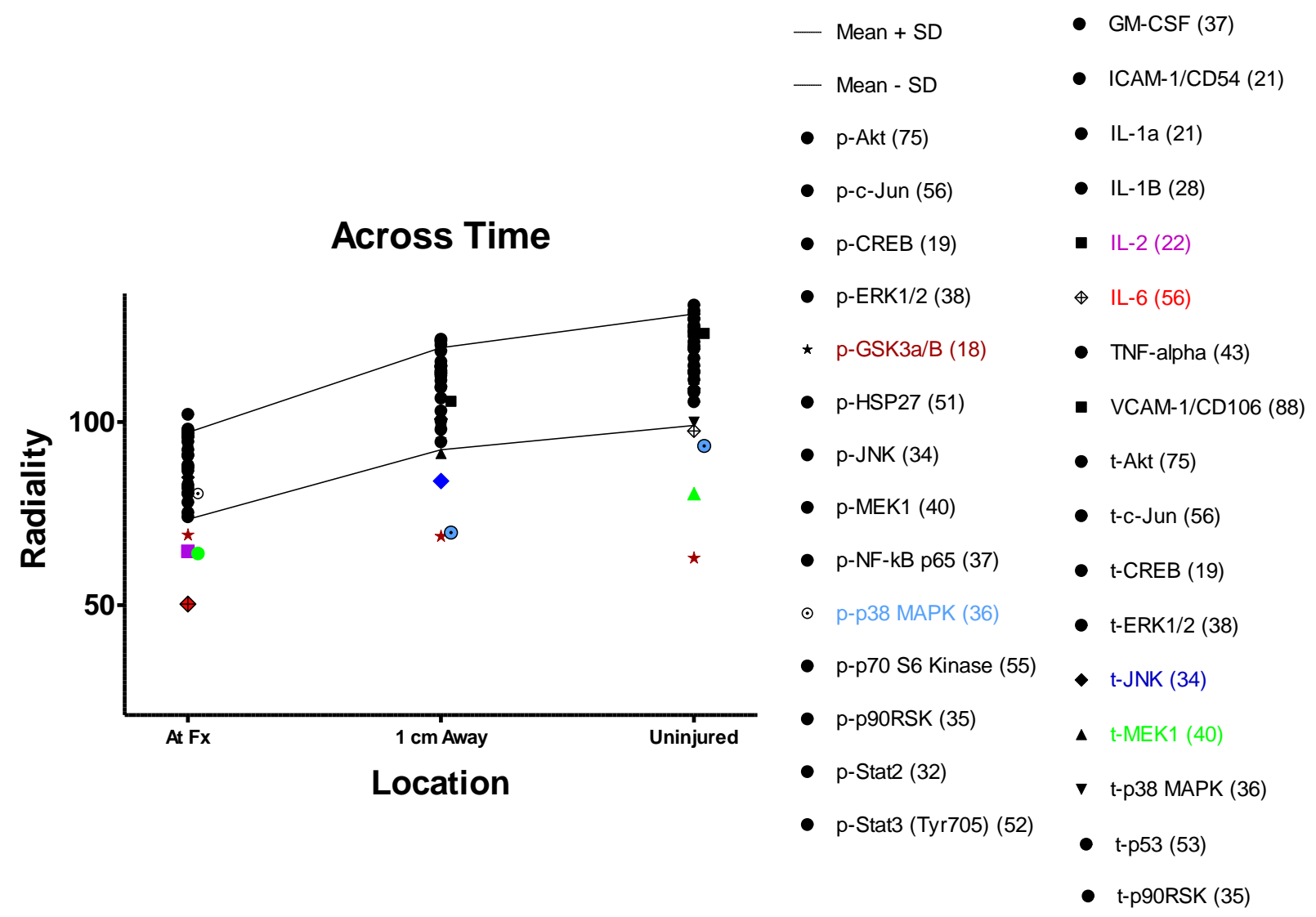

Figure 4-10. Across time points radiality. Radiality values were calculated based on protein responses at all 4 time points. Proteins with radiality less than the mean observed radiality minus the standard deviation are shown in color.

\subsection{Discussion}

\subsubsection{Spatial Protein Responses to Injury}

The current understanding of the molecular level changes that occur during tissue injury and repair is extremely limited. Specifically, little is known regarding the spatial differences in molecular responses following traumatic injury. The most well characterized aspect of the response to traumatic injury is the regulatory role of cytokines. The temporal cytokine response 
to traumatic injury and other physical stresses has been extensively studied in various tissues and experimental models. $^{22,}{ }^{46-53}$ Cytokine production reflects the degree of tissue trauma. ${ }^{3}$ Temporal changes in cytokine concentrations have been shown to correlate with negative early post-trauma implications such as acute respiratory distress syndrome (ARDS), multiple organ failure (MOF), wound failure, and mortality. ${ }^{49,}$ 54-56 Temporal cytokine responses to traumatic injuries have demonstrated utility as objective predictors of acute wound healing outcomes that can assist surgeons in making appropriate surgical decisions with regard to procedural selection and timing. ${ }^{49-53}$

Since the heavily studied temporal cytokine responses have shown promise as predictive biomarkers beneficial to the treatment of traumatic wounds, knowledge of spatial differences in molecular responses to injury may also be beneficial to the treatment of wounds. Previous studies have determined that the local immune environment of traumatic injury sites differ in cytokine concentration in comparison to systemically circulating concentrations, ${ }^{22}$ but the relationship between tissue cytokine concentrations and physical distance from the injury site has not been elucidated. If cytokines display spatial relationships to injury sites, they may be used as quantitative descriptors of the state of different regions of tissue following traumatic injury. The results obtained in this work identified the existence of spatial cytokine concentration gradients in response to blunt trauma using a reproducible injury model.

The cytokines assayed showed significant spatial differences in concentration at all of the studied time points with the majority of significant differences being observed during the early response to injury (immediately following the injury and $6 \mathrm{~h}$ after the injury). Immediately following the injury MIP-1a and IL-2 showed elevated levels at the injury site, and $6 \mathrm{~h}$ after the injury, IL-6, IL-1ß, and MIP-1a levels were elevated at the injury site. Of all the cytokines 
assayed, IL-6 showed the strongest spatial relationship to the zone of injury. At the $6 \mathrm{~h}$ time point IL-6 levels were significantly different at all 3 sampling locations. At this time point, IL-6 levels decreased as distance from the fracture increased suggesting that IL- 6 could potentially be used as a spatial biomarker of the zone of injury. IL-6 is well established as an important modulator of the chain of events that follow traumatic injury, and has been related to negative patient outcome following trauma. ${ }^{55}$ IL-6 has also been shown to induce satellite cell proliferation. $^{40}$ The IL-6 response observed in this work is in general agreement with previous studies; Hauser, et al. showed that IL-6 levels peaked before $48 \mathrm{~h}$ and then decreased in softtissue hematoma sera of humans with high-energy bone fractures. ${ }^{22}$ Pape, et al. observed a peak in circulating IL-6 levels $7 \mathrm{~h}$ after skeletal fracture surgery. ${ }^{52}$ They described IL-6 as a pivotal marker of the response to blunt trauma, and demonstrate that increased levels are indicative of surgical complications and adverse outcomes. ${ }^{52}$

In addition to cytokines, the spatial relationship of MAPKs to the injury site was also investigated. MAPKs are involved in the signaling downstream of cytokine receptors, and the production of some cytokines can be regulated by MAPKs. ${ }^{57}$ Tissue samples were analyzed to determine if spatial differences in the levels of phosphorylated MAPKs also exist. Immediately following the femur fracture 8 of the 14 MAPKs assayed had spatially different levels of phosphorylated protein at the different sampling locations. At this earliest time point phosphorylated Akt, c-Jun, ERK1/2, GSK-3 $\alpha / 3$, HSP27, p70S6, and STAT3 levels were significantly higher in the uninjured leg than in the samples taken at the fracture site and $1 \mathrm{~cm}$ away. The phosphorylated levels measured in samples at the fracture site and in samples $1 \mathrm{~cm}$ away from the fracture were not significantly different from each other for these 7 phosphorylated proteins. This indicates that the tissue at the fracture site has a similar response 
to the tissue $1 \mathrm{~cm}$ away with regard to these proteins. This also indicates that immediately following injury phosphorylation activity generally decreases at the fracture site and $1 \mathrm{~cm}$ away, as there were no instances where phosphorylated protein levels were higher at these two sites than in the uninjured tissue. This decrease in phosphorylation may be due to loss of cellular regulation over kinase activity that may arise as a result of the breakdown of the cellular cytoskeleton leading to disruption of scaffold proteins that help facilitate kinase interactions and loss of spatial kinase separation. ${ }^{58}$ The decrease in phosphorylation activity could also be due to ischemia and impaired/injured mitochondria leading to reduced ATP availability which is necessary for phosphorylation to occur.

Six $\mathrm{h}$ after the femur fracture only 3 of the 14 assayed proteins displayed spatial differences in phosphorylated protein levels. These phosphorylated proteins were MEK1, GSK$3 \alpha / \beta$, and Akt. Twenty-four $\mathrm{h}$ after the femur fracture, GSK-3 $\alpha / \beta$ and $\mathrm{p} 38$ were the only phosphorylated proteins that showed significant differences between locations, and p38, MEK1, and p70S6 were the only phosphorylated proteins with levels that were significantly different when comparing the different sampling locations at the $168 \mathrm{~h}$ time point. Overall, these results suggest that MEK1, GSK-3 $\alpha / \beta$, Akt, p38, and p70S6 are likely to play key roles in mediating the later tissue response to injury. Akt, p70S6, STAT3, and MEK1 have previously been implicated as proteins important to cytokine signaling, specifically in IL-2 mediated signaling. ${ }^{8}$ GSK-3 $\alpha / \beta$ is known to be an extremely important mediator of muscle tissue physiology, ${ }^{29}$ and p38 is a well known responder to cytokines and many different types of stress. ${ }^{59-61}$

In addition to phosphorylated proteins and cytokines, the total levels (phosphorylated or not) for some of the phosphoproteins were also measured. Immediately following the femur fracture, only two proteins (Akt and ERK1/2) were found to have significantly different levels in 
total protein at the different sampling locations both having higher levels in the uninjured leg than at the fracture site and $1 \mathrm{~cm}$ away. Only p38 levels were significantly different across locations $6 \mathrm{~h}$ after the injury. p38 and MEK1 were the only proteins with significantly different levels across sampling locations $24 \mathrm{~h}$ after fracture. The most spatial differences in total protein levels were found $168 \mathrm{~h}$ after the injury with ERK1/2, p38, Akt, CREB and JNK all showing spatial differences in total protein levels. All of the spatial differences observed at this time point show increased total protein expression at the fracture site, reflecting phenotypic differences in newly healed or grown tissue in comparison to undisturbed tissue in the uninjured leg. Ischemia is known to activate MAPK signaling, and both ERK1/2 and p38 signaling can activate CREB in response to stress. ${ }^{58}$

In summary, the protein levels measured indicate that IL- 2 and MIP-1 $\alpha$ may be key to initiating the tissue response to injury. The most significant variations in cytokine levels across locations are observed at the $6 \mathrm{~h}$ time point, and differences in total protein levels across sampling locations are observed primarily $168 \mathrm{~h}$ after injury. Overall, the data indicates that with regard to the proteins studied, immediately following injury, phosphoproteins may be the most suitable biomarkers of the zone of injury, while 6 hours after injury cytokines are better suited. This is the first study to analyze the levels of phosphorylated proteins in tissue samples at different distances from a traumatic injury. It provides evidence that the monitoring of phosphorylated proteins could potentially be used as a spatial marker of the state of injured tissue. These results demonstrate that spatial cytokine and MAPK gradients exist following traumatic injury, representing potential biomarkers that may be used to define the ZOI. 


\subsubsection{Network Response to Tissue Injury}

The data presented here, as well as in other studies, have made it clear that cytokines are essential mediators of the injury response. ${ }^{10,12}$ Even with the multitude of existing data describing signaling pathways downstream of cytokines, the means by which cells and tissues integrate multiple cytokine signals to achieve desired responses is unclear. MAPKs are downstream responders to cytokine signaling and essential to stress responses. However, the networked relationships between cytokines and MAPKs following traumatic injury remains poorly understood. The observed individual protein responses presented here demonstrate how challenging it is to understand how multiple proteins function together when responding to a stress as complex as traumatic tissue injury. The network analysis technique developed in this body of work was applied to the individual cytokine, phosphorylated MAPK, and total MAPK responses to provide an integrated view of how these multiple components function collectively as a network in response to tissue injury. The analysis describes the network cohesiveness with which each individual protein responds to the injury. A protein with low radiality is responding to the injury in a different way than the majority of other proteins, and may be important to the injury response in muscle. Comparison of the radiality values obtained at different time points and sampling locations can reveal how protein network roles change in response to varying degrees of perturbation, and assist in defining network relationships involved in muscle healing. Figure 4-11 summarizes the network analysis results by mapping the time course of key proteins to the tissue response at each location. 
$\underline{\text { Time Post Injury }}$

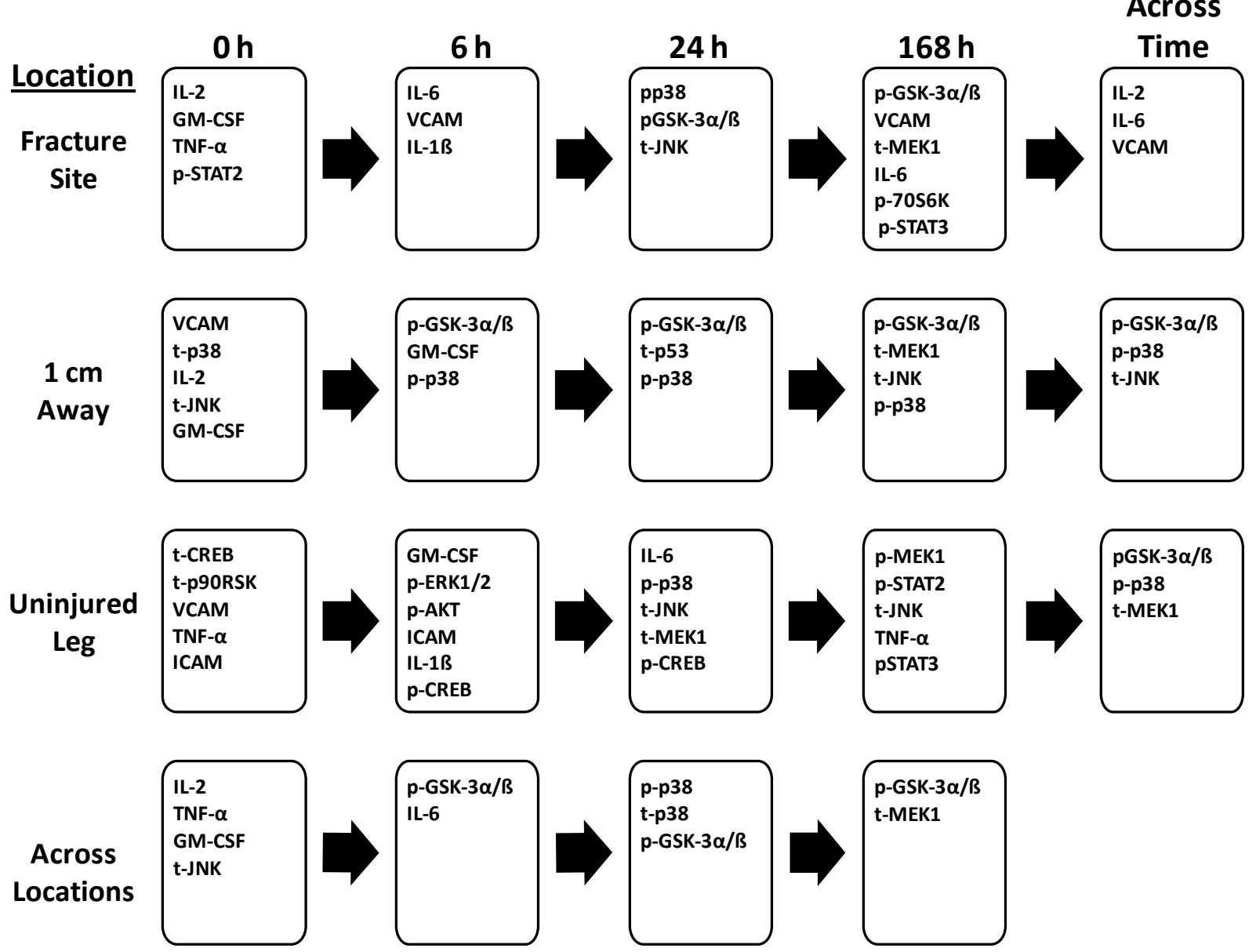

Figure 4-11. Schematic depicting important proteins identified at each time point and sampling location. The depicted proteins each had radiality values less than the mean radiality value minus the standard deviation of the mean.

STAT2 was the only phosphorylated protein identified by the analysis at the earliest time point for any of the network analyses, including the across locations analysis. STAT proteins are known as important downstream rapid responders of cytokine signals. ${ }^{62,63}$ Research has shown that STAT2 is required for early differentiation of satellite cells in injury-induced skeletal muscle regeneration. ${ }^{34}$ For the network at the fracture site, IL-2 radiality had the lowest radiality of all the proteins immediately following femur fracture. It is well known that IL-2 can activate STAT proteins, and STAT proteins can actually dock onto the intracellular portion of the IL-2 
receptor. ${ }^{8,62,64}$ Simple inspection or statistical analysis of the raw phosphorylation data does not provide any indication of such a relationship. The network analysis also identified IL-2 as an important responder immediately following injury in the networks constructed $1 \mathrm{~cm}$ away from the injury, in the across locations network, and in the across time points analysis at the fracture site.

An additional known reaction that occurs immediately following injury is the release of TNF- $\alpha$ which in turn stimulates the release of IL-6. ${ }^{3,13}$ The network analysis at the fracture site as well as the across locations network analysis identified TNF- $\alpha$ as an important protein immediately following the injury, and IL-6 subsequently emerged as an important protein $6 \mathrm{~h}$ following the injury for the fracture site analysis and across locations analysis. IL-6 is the main cytokine responsible for inducing systemic changes known as the acute phase response. ${ }^{9,} 65$ TNF- $\alpha$ and IL-6 are well known regulators of muscle integrity, ${ }^{11,13,17,26,66}$ and have been shown to function as inhibitors of myogenesis. ${ }^{67-70}$ During inflammatory responses, these two proteins have been shown to inhibit repair processes, promoting protein degradation and the breakdown of existing tissue. ${ }^{13,26,69,70}$

Much of the network analysis results point to GSK-3 $\alpha / \beta$ phosphorylation activity playing a significant role in the muscle tissue response to injury. This agrees with a growing amount of evidence that implicates GSK-3 $\alpha / \beta$ as a central regulator of skeletal muscle plasticity. ${ }^{16,29,}$,11-73 Phosphorylated GSK-3 $\alpha / \beta$ was identified as a key protein in the network analysis results $24 \mathrm{~h}$ after the injury at the fracture site; in the network analysis results $1 \mathrm{~cm}$ away from the injury at the 6,24 , and $168 \mathrm{~h}$ time points; in the across locations network analysis results at the 6,24 , and $168 \mathrm{~h}$ time points; and by the across time points analysis $1 \mathrm{~cm}$ away and in the uninjured leg. GSK-3 $\alpha / \beta$ has been referred to as a "master regulator" of skeletal muscle, having an influence on 
atrophy, protein degradation, protein synthesis, and cell death in muscle cells. ${ }^{29}$ The results obtained in this work show decreased phosphorylation of GSK-3 $\alpha / 3$ on Ser-21/9 at the fracture site in comparison to the uninjured leg at the 0,6 , and $24 \mathrm{~h}$ time points. It is important to note that a decrease in Ser-21/9 phosphorylation on GSK-3 $\alpha / \beta$ corresponds to an increase in GSK$3 \alpha / \beta$ activity. ${ }^{29}$ Previous studies have also observed increased GSK-3 $\alpha / \beta$ activity (phosphorylation on Ser-21/9) in burn injury induced atrophying muscle. ${ }^{71,72}$ Pharmacological inhibition of GSK3 with TDZD-8 has even been used to block burn induced muscle protein breakdown. $^{71}$ The network analysis approach was able to reflect the importance of GSK-3 $\alpha / \beta$ activity to the complex network regulation of the tissue level response to injury based on condition specific data.

GSK-3 $\alpha / \beta$ has been implicated as a downstream target of the Akt/mammalian target of rapamycin (mTOR) signaling pathway following its activation during muscle remodeling. ${ }^{16}$ Akt inhibits GSK-3 $\alpha / \beta$ activity directly by phosphorylating Ser- 21 and Ser- 9 on the $\alpha$ and $\beta$ isoforms, respectively. ${ }^{73-76}$ At the 0 and $6 \mathrm{~h}$ time points both Akt phosphorylation and GSK-3 $\alpha / 3$ phosphorylation (measured on Ser-21 for GSK-3 $\alpha$ and on Ser-9 for GSK-3ß) were significantly less at the fracture site in comparison to the uninjured leg. Akt functioning is essential in skeletal muscle for myogenesis and proper maintenance of muscle mass. ${ }^{26}$ The Akt signaling pathway promotes protein synthesis to increase muscle mass partly through the simultaneous activation of p70S6K and inactivation of GSK-3 $\alpha / \beta .{ }^{16,26}$ Attenuated Akt signaling results in increased GSK$3 \alpha / \beta$ activity (decreased Ser-21/9 phosphorylation) in skeletal muscle, which in turn contributes to enhanced protein degradation. ${ }^{29}$ Akt phosphorylation is diminished in many muscular disease states, ${ }^{16,73}$ and a study by Bodine et al. demonstrated that both Akt and GSK-3ß phosphorylation increase in rat models during compensatory muscle hypertrophy (growth and size increase). ${ }^{77}$ 
While a study by Smith et al. found that reduced Akt activity was accompanied by reduced phosphorylation of GSK-3ß in muscles with increased protein degradation. ${ }^{78}$ The results presented in this work show that Akt activity decreases in response to the injury while GSK-3 $\alpha / \beta$ activity increases, suggesting that the injured tissue is not attempting to synthesize proteins or promote growth as much as the uninjured tissue. Taken together, the identification of IL-6 and TNF- $\alpha$ as important proteins at the early time points as well as the decreased Akt and GSK3- $\alpha / \beta$ phosphorylation, suggest that at the early time points cellular efforts are directed towards stopping growth and breaking down existing tissue.

Many of the network analyses pointed to p38 as an important protein in the injury response. Phosphorylated p38 was identified as an important protein at the $24 \mathrm{~h}$ time point by all of the network analyses (all 3 locations and across locations). Phosphorylated p38 was also highlighted by the analysis $1 \mathrm{~cm}$ away at the $168 \mathrm{~h}$ time point and by the across time points analysis $1 \mathrm{~cm}$ away and in the uninjured leg. Total p38 was highlighted by the $0 \mathrm{~h}, 1 \mathrm{~cm}$ away analysis and by the $24 \mathrm{~h}$ across locations analysis. p38 is a known mediator of myogenic differentiation. ${ }^{79-84} \mathrm{p} 38$ is recognized as a necessary switch that turns on differentiation, ${ }^{79,82,84}$ with p38 activity having been shown to increase dramatically during myoblast myogenic differentiation $^{80,83}$ and in injured human muscle..$^{85}$ p38 activation has been shown to occur 1 day after muscle injury and last as long as 10 days. ${ }^{17}$ The results obtained here align with this previous study, with high p38 levels being observed $24 \mathrm{~h}$ and $168 \mathrm{~h}$ after the injury, and suggest that at these time points p38 is an essential coordinator of network efforts that are more directed towards differentiation of cells to promote healing. 


\subsubsection{Potential Bystander Effects}

The results of this study may be relatable to the bystander effect. The bystander effect is typically referred to as the observation of similar deleterious effects or even death of unexposed cells, when neighbor cells have been affected by some injurious event. ${ }^{86}$ The effects observed in neighboring cells are believed to be due to signals that affected cells release as a result of changes in their gene expression induced by the injury. ${ }^{86}$ The most well studied injurious event is that of radiation exposure. ${ }^{87}$ The bystander effect is also observed following transfection, as transfected cells have been shown to transfer death signals to their neighboring cells. ${ }^{88}$ The bystander effect has also been associated with chemical exposures. ${ }^{89,90}$

While radiation, transfection, and chemically induced bystander effects have been extensively studied, the notion that similar bystander effect mechanisms may occur in physically induced traumatic tissue injury has received little attention. Tissue functioning is greater than the sum of its cellular parts. ${ }^{91}$ Individual cells are directed in large part by extracellular signaling that mediates cell-to-cell communication as well as the interaction of cells with the extracellular matrix. These interactions are modulated by the tissue microenvironment, where cytokines predominate..$^{91}$ Extracellular signaling is responsible for the integration of multi-cellular damage responses, and the bystander effect may simply be the result of this extracellular signaling that modulates cellular repair and death programs. ${ }^{15}$ The bystander effect may also occur in the case of physical traumatic injury. The data obtained in this work are suggestive of this, as similar protein responses were often obtained when comparing the fracture site to $1 \mathrm{~cm}$ away. These particular protein responses were not the same as the responses in the uninjured leg. These similar responses were most frequently observed for phosphorylated protein responses at the $0 \mathrm{~h}$ time point. Immediate responses are coordinated by phosphorylation, and cytokines and MAPKs 
have been implicated as mediators of the bystander effect. ${ }^{87,88,90,92}$ Akt and GSK-3 $\alpha / \beta$ were among the phosphorylated proteins with similar responses at the fracture site and $1 \mathrm{~cm}$ away. Previous research has associated the Akt-GSK-3 $\alpha / \beta$ pathway with bystander effect signals. ${ }^{93}$

The analysis identified total MEK1 as an important protein to the $168 \mathrm{~h}$ tissue response at the fracture site, $1 \mathrm{~cm}$ away, and by the across locations analysis. In the uninjured analysis total MEK1 was highlighted at the $24 \mathrm{~h}$ time point and phosphorylated MEK1 was highlighted at the 168 time point. MEK1 was also highlighted by the across time points analysis for the uninjured leg. MEK1 is recognized as a necessary regulator of myogenesis in skeletal muscle. Whether or not MEK1 signaling has a stimulatory or inhibitor effect on myogenesis depends on the specific stage of healing process. ${ }^{94}$

Due to their involvement in the regulation of inflammatory mediators MAPKs, GSK-3 $\alpha / \beta$ and p38 in specific, have received attention as therapeutic targets for the treatment of a variety of conditions. $^{73,95,96}$ These two proteins were heavily indicated as being important to the tissue response by the analysis performed in this work due to their identification at multiple time points and locations. This demonstrates the potential utility of the presented approach as an initial screen to identify potential therapeutic targets.

\subsection{Conclusions}

This study has shown that following traumatic injury, key markers of wound healing display spatial concentration gradients with regard to the injury site. These gradients represent biomarkers that may be used to define the ZOI, and aid in the spatial assessment of the state of injured tissue. The cytokines and MAPKs studied here represent biomarkers worth pursuing in 
the development of clinical assays aimed to guide the effective assessment and treatment of wounds.

It can be seen that it is difficult to understand and represent integrated stress responses of tissue signaling networks, even with an incomplete coverage of just a few proteins present in muscle tissue. The analysis presented here sheds light on the network mechanisms involved in the muscle response to injury, mapping dynamic condition specific relationships over both time and space. The analysis revealed IL-6 as an important spatial marker of the ZOI, and revealed GSK-3 $\alpha / ß$ as an extremely important protein in the muscle injury response to blunt force trauma. Some of the results obtained here align with previous research studies in identifying the same proteins as important to muscle tissue response, while other proteins identified by the analysis have not previously been recognized as being important to the response. The vast majority of studies investigating the specific roles of proteins under injurious inflammatory inducing conditions are performed in vitro, typically focusing on the up or down regulation of a few select proteins. Specifically, much of the current knowledge regarding how cytokines affect intracellular signaling comes from studies of isolated immune cells. Different cells can respond differently to the same cytokines. The results presented here are unique in that they reflect the entire tissue (consisting of both immune cells and muscle cells) response to trauma, and reflect the networked response of multiple proteins across varying degrees of perturbation. Current technology does not yet permit us to monitor in real time all of the interactions occurring between signaling molecules in living organisms and directly visualize the structure and dynamics of the network they comprise. The network analysis approach presented in this work can help fill this gap by providing an integrated data-driven description of how biological networks respond to stress. 


\subsection{Future Directions}

The millions of molecules comprising entire organisms can be considered as one large signaling network. The network analysis approach presented here only captures a small portion of this expansive network. In the future, more biological information could be extracted by monitoring more protein targets, and by incorporating additional network components such as hormones, mRNA, and scaffolding proteins in the analysis. In this analysis, the only type of post translational modification monitored was that of phosphorylation and only at one site on each target protein. Many proteins can undergo a multiple types of post-translational modifications and at multiple amino acid sites. The number and diversity of nodes included in the network analysis can be expanded to include these modifications and provide richer biological information pertaining to any problem of interest. The developed approach can easily be applied to larger datasets, and is only limited by the technology used to simultaneously detect node targets. Additionally, this work focused on a single graph theory parameter. There are many different graph theory parameters, and even more are sure to be developed in the future. Different graph theory parameters can be used to provide unique interpretations and perspective. In future work, the utility additional centrality parameters will be explored.

Spatial molecular response comparisons were made in this work to identify objective predictors of acute wound healing outcomes that could potentially be used in point of care assays to assist surgeons in making appropriate surgical decisions. Following traumatic injury the boundaries of viable tissue are of particular importance to surgeons who must decide the proper regions of debridement. If too little tissue, is removed patients will need to be subjected to repeated costly and painful surgical procedures. If too much tissue is removed, optimal healing and recovery may be hindered. Currently, the assessment of viable tissue in the operating room 
is entirely based on visual inspection of the injured tissue. An approach to predict cell death by monitoring the early phosphorylation of key proteins has been developed by Vrana et al. ${ }^{97}$ This approach has currently only been applied to predict cell death in vitro following chemical exposure. The application of this approach to phosphorylation responses obtained from injured muscle tissue could provide the basis for developing point of care assays to improve surgical decision making. 


\subsection{Bibliography}

(1) Bentzinger, C. F., Wang, Y. X., and Rudnicki, M. A. (2012) Building muscle: molecular regulation of myogenesis. Cold Spring Harb Perspect Biol, 4, 1-16.

(2) Lopez, A. F., Hercus, T. R., Ekert, P., Littler, D. R., Guthridge, M., Thomas, D., Ramshaw, H. S., Stomski, F., Perugini, M., D'Andrea, R., Grimbaldeston, M., and Parker, M. W. (2010) Molecular basis of cytokine receptor activation. IUBMB Life, 62, 509-518.

(3) Desborough, J. P. (2000) The stress response to trauma and surgery. Br J Anaesth, 85, 109-117.

(4) Dinarello, C. A. (2007) Historical Review of Cytokines. Eur J Immunol, 37, S34-45.

(5) Dong, C., Davis, R. J., and Flavell, R. A. (2002) MAP kinases in the immune response. Annu Rev Immunol, 20, 55-72.

(6) Guthridge, M. A., Powell, J. A., Barry, E. F., Stomski, F. C., McClure, B. J., Ramshaw, H., Felquer, F. A., Dottore, M., Thomas, D. T., To, B., Begley, C. G., and Lopez, A. F. (2006) Growth factor pleiotropy is controlled by a receptor Tyr/Ser motif that acts as a binary switch. Embo J, 25, 479489.

(7) Ozaki, K., and Leonard, W. J. (2002) Cytokine and Cytokine Receptor Pleiotropy and Redundancy. J. Biol. Chem., 277, 29355-29358.

(8) Leonard, W. J., and Lin, J. X. (2000) Cytokine receptor signaling pathways. J Allergy Clin Immunol, $105,877-888$.

(9) Werner, S., and Grose, R. (2003) Regulation of wound healing by growth factors and cytokines. Physiol Rev, 83, 835-870.

(10) Barrientos, S., Stojadinovic, O., Golinko, M. S., Brem, H., and Tomic-Canic, M. (2008) Growth factors and cytokines in wound healing. Wound Repair Regen, 16, 585-601.

(11) Reid, M. B., and Li, Y. P. (2001) Cytokines and oxidative signalling in skeletal muscle. Acta Physiol Scand, 171, 225-232. 
(12) Neher, M., Weckbach, S., Flierl, M., Huber-Lang, M., and Stahel, P. (2011) Molecular mechanisms of inflammation and tissue injury after major trauma--is complement the "bad guy"? J Biomed Sci, 18, 90-106.

(13) Zoico, E., and Roubenoff, R. (2002) The role of cytokines in regulating protein metabolism and muscle function. Nutr Rev, 60, 39-51.

(14) Tidball, J. G. (2005) Inflammatory processes in muscle injury and repair. Am J Physiol Regul Integr Comp Physiol, 288, R345-353.

(15) Barcellos-Hoff, M. H. (1998) How do tissues respond to damage at the cellular level? The role of cytokines in irradiated tissues. Radiat Res, 150, S109-120.

(16) Bassel-Duby, R., and Olson, E. N. (2006) Signaling pathways in skeletal muscle remodeling. Annu Rev Biochem, 75, 19-37.

(17) Chen, S. E., Gerken, E., Zhang, Y., Zhan, M., Mohan, R. K., Li, A. S., Reid, M. B., and Li, Y. P. (2005) Role of TNF-alpha signaling in regeneration of cardiotoxin-injured muscle. Am J Physiol Cell Physiol, 289, C1179-1187.

(18) Brem, H., Stojadinovic, O., Diegelmann, R. F., Entero, H., Lee, B., Pastar, I., Golinko, M., Rosenberg, H., and Tomic-Canic, M. (2007) Molecular Markers in Patients with Chronic Wounds to Guide Surgical Debridement. Mol Med, 13, 30-39.

(19) Bryan, D., Walker, K. B., Ferguson, M., and Thorpe, R. (2005) Cytokine gene expression in a murine wound healing model. Cytokine, 31, 429-438.

(20) Hietbrink, F., Koenderman, L., Rijkers, G., and Leenen, L. (2006) Trauma: the role of the innate immune system. World J Emerg Surg, 1, 15.

(21) Faries, P. L., Marin, M. L., Veith, F. J., Ramirez, J. A., Suggs, W. D., Parsons, R. E., Sanchez, L. A., and Lyon, R. T. (1996) Immunolocalization and temporal distribution of cytokine expression 
during the development of vein graft intimal hyperplasia in an experimental model. J Vasc Surg, $24,463-471$.

(22) Hauser, C. J., Zhou, X., Joshi, P., Cuchens, M. A., Kregor, P., Devidas, M., Kennedy, R. J., Poole, G. V., and Hughes, J. L. (1997) The immune microenvironment of human fracture/soft-tissue hematomas and its relationship to systemic immunity. J Trauma, 42, 895-904.

(23) Nagaraju, K., Raben, N., Merritt, G., Loeffler, L., Kirk, K., and Plotz, P. (1998) A variety of cytokines and immunologically relevant surface molecules are expressed by normal human skeletal muscle cells under proinflammatory stimuli. Clin Exp Immunol, 113, 407-414.

(24) Summan, M., McKinstry, M., Warren, G. L., Hulderman, T., Mishra, D., Brumbaugh, K., Luster, M. I., and Simeonova, P. P. (2003) Inflammatory mediators and skeletal muscle injury: a DNA microarray analysis. J Interferon Cytokine Res, 23, 237-245.

(25) Wang, M., Wang, Q., Zhang, X., Yang, Y., Zhao, H., Ma, Y., and Pan, Y. (2011) Uncovering the transcriptional circuitry in skeletal muscle regeneration. Mamm Genome, 22, 272-281.

(26) Guttridge, D. (2004) Signaling pathways weigh in on decisions to make or break skeletal muscle. Curr Opin Clin Nutr Metab Care, 4, 443-450.

(27) Brooks, S. V. (2003) Current topics for teaching skeletal muscle physiology. Adv Physiol Educ, 27, 171-182.

(28) Williams, R., and Neufer, P. (1996) Exercise: Regulation and Integration of Multiple Systems, In The Handbook of Physiology (Rowell, L., and Shepherd, J., Eds.) pp 1124-1150, Am Physiol Soc, Bethesda, MD.

(29) Verhees, K. J. P. (2013) No time to waste: a glycogen synthase kinase-3ß-glucocorticoid receptor signaling axis illuminated, In Graduate School VLAG p 287, Wageningen University, the Netherlands. 
(30) Hohlfeld, R., and Engel, A. G. (1994) The immunobiology of muscle. Immunol Today, 15, 269274.

(31) Wiendl, H., Hohlfeld, R., and Kieseier, B. C. (2005) Immunobiology of muscle: advances in understanding an immunological microenvironment. Trends Immunol, 26, 373-380.

(32) Curfs, J. H., Meis, J. F., and Hoogkamp-Korstanje, J. A. (1997) A primer on cytokines: sources, receptors, effects, and inducers. Clin Microbiol Rev, 10, 742-780.

(33) Yin, H., Price, F., and Rudnicki, M. A. (2013) Satellite cells and the muscle stem cell niche. Physiol Rev, 93, 23-67.

(34) Wang, K., Wang, C., Xiao, F., Wang, H., and Wu, Z. (2008) JAK2/STAT2/STAT3 are required for myogenic differentiation. J Biol Chem, 283, 34029-34036.

(35) Beauchamp, J. R., Morgan, J. E., Pagel, C. N., and Partridge, T. A. (1999) Dynamics of myoblast transplantation reveal a discrete minority of precursors with stem cell-like properties as the myogenic source. J Cell Biol, 144, 1113-1122.

(36) Bischoff, R. (1990) Interaction between satellite cells and skeletal muscle fibers. Development, $109,943-952$.

(37) Kuang, S., Kuroda, K., Le Grand, F., and Rudnicki, M. A. (2007) Asymmetric self-renewal and commitment of satellite stem cells in muscle. Cell, 129, 999-1010.

(38) Robertson, T. A., Maley, M. A., Grounds, M. D., and Papadimitriou, J. M. (1993) The role of macrophages in skeletal muscle regeneration with particular reference to chemotaxis. Exp Cell Res, 207, 321-331.

(39) Chazaud, B., Sonnet, C., Lafuste, P., Bassez, G., Rimaniol, A. C., Poron, F., Authier, F. J., Dreyfus, P. A., and Gherardi, R. K. (2003) Satellite cells attract monocytes and use macrophages as a support to escape apoptosis and enhance muscle growth. J Cell Biol, 163, 1133-1143. 
(40) Cantini, M., and Carraro, U. (1995) Macrophage-released factor stimulates selectively myogenic cells in primary muscle culture. J Neuropathol Exp Neurol, 54, 121-128.

(41) Stahel, P. F., Smith, W. R., and Moore, E. E. (2007) Role of biological modifiers regulating the immune response after trauma. Injury, 38, 1409-1422.

(42) Lindsey, B. A., Clovis, N. B., Smith, E. S., Salihu, S., and Hubbard, D. F. (2010) An animal model for open femur fracture and osteomyelitis: Part I. J Orthop Res, 28, 38-42.

(43) Al-Mousawi, A. M., Kulp, G. A., Branski, L. K., Kraft, R., Mecott, G. A., Williams, F. N., Herndon, D. N., and Jeschke, M. G. (2010) Impact of anesthesia, analgesia, and euthanasia technique on the inflammatory cytokine profile in a rodent model of severe burn injury. Shock, 34, 261-268.

(44) Hulse, R. E., Kunkler, P. E., Fedynyshyn, J. P., and Kraig, R. P. (2004) Optimization of multiplexed bead-based cytokine immunoassays for rat serum and brain tissue. J Neurosci Methods, 136, 8798.

(45) Currie, H. N., Loos, M., Vrana, J., Dragan, K., and Boyd, J. (2014) Spatial cytokine distribution following traumatic injury. Cytokine, 66, 112-118.

(46) Hisano, S., Sakamoto, K., Ishiko, T., Kamohara, H., and Ogawa, M. (1997) IL-6 and soluble IL-6 receptor levels change differently after surgery both in the blood and in the operative field. Cytokine, 9, 447-452.

(47) Maier, B., Lefering, R., Lehnert, M., Laurer, H. L., Steudel, W. I., Neugebauer, E. A., and Marzi, I. (2007) Early versus late onset of multiple organ failure is associated with differing. Shock, 28, 668-674.

(48) Martin, C., Boisson, C., Haccoun, M., Thomachot, L., and Mege, J. L. (1997) Patterns of cytokine evolution (tumor necrosis factor-alpha and interleukin-6). Crit Care Med, 25, 1813-1819. 
(49) Hawksworth, J. S., Stojadinovic, A., Gage, F. A., Tadaki, D. K., Perdue, P. W., Forsberg, J., Davis, T. A., Dunne, J. R., Denobile, J. W., Brown, T. S., and Elster, E. A. (2009) Inflammatory biomarkers in combat wound healing. Ann Surg, 250, 1002-1007.

(50) Jiang, Z., Berceli, S. A., Pfahnl, C. L., Wu, L., Goldman, D., Tao, M., Kagayama, M., Matsukawa, A., and Ozaki, C. K. (2004) Wall shear modulation of cytokines in early vein grafts. J Vasc Surg, 40, 345-350.

(51) Pape, H. C., Schmidt, R. E., Rice, J., van Griensven, M., das Gupta, R., Krettek, C., and Tscherne, H. (2000) Biochemical changes after trauma and skeletal surgery of the lower extremity: quantification of the operative burden. Crit Care Med, 28, 3441-3448.

(52) Pape, H. C., van Griensven, M., Rice, J., Gansslen, A., Hildebrand, F., Zech, S., Winny, M., Lichtinghagen, R., and Krettek, C. (2001) Major secondary surgery in blunt trauma patients and perioperative cytokine. J Trauma, 50, 989-1000.

(53) Strecker, W., Gebhard, F., Perl, M., Rager, J., Buttenschon, K., Kinzl, L., and Beck, A. (2003) Biochemical characterization of individual injury pattern and injury severity. Injury, 34, 879-887.

(54) Biffl, W. L., Moore, E. E., Moore, F. A., and Peterson, V. M. (1996) Interleukin-6 in the injured patient. Marker of injury or mediator of inflammation? Ann Surg, 224, 647-664.

(55) Meduri, G. U., Headley, S., Kohler, G., Stentz, F., Tolley, E., Umberger, R., and Leeper, K. (1995) Persistent elevation of inflammatory cytokines predicts a poor outcome in ARDS. Plasma IL-1 beta and IL- 6 levels are consistent and efficient predictors of outcome over time. Chest, 107, 1062-1073.

(56) Roumen, R. M., Hendriks, T., van der Ven-Jongekrijg, J., Nieuwenhuijzen, G. A., Sauerwein, R. W., van der Meer, J. W., and Goris, R. J. (1993) Cytokine patterns in patients after major vascular surgery, hemorrhagic shock, and severe blunt trauma. Relation with subsequent adult respiratory distress syndrome and multiple organ failure. Ann Surg, 218, 769-776. 
(57) Liu, Y., Shepherd, E. G., and Nelin, L. D. (2007) MAPK phosphatases--regulating the immune response. Nat Rev Immunol, 7, 202-212.

(58) Irving, E. A., and Bamford, M. (2002) Role of mitogen- and stress-activated kinases in ischemic injury. J Cereb Blood Flow Metab, 22, 631-647.

(59) Cargnello, M., and Roux, P. P. (2011) Activation and Function of the MAPKs and Their Substrates, the MAPK-Activated Protein Kinases. Microbiol Mol Biol Rev, 75, 50-83.

(60) Xiao, Y. Q., Malcolm, K., Worthen, G. S., Gardai, S., Schiemann, W. P., Fadok, V. A., Bratton, D. L., and Henson, P. M. (2002) Cross-talk between ERK and p38 MAPK mediates selective suppression of pro-inflammatory cytokines by transforming growth factor-beta. J Biol Chem, 277, 1488414893.

(61) Lee, J. C., Laydon, J. T., McDonnell, P. C., Gallagher, T. F., Kumar, S., Green, D., McNulty, D., Blumenthal, M. J., Keys, J. R., Land vatter, S. W., Strickler, J. E., McLaughlin, M. M., Siemens, I. R., Fisher, S. M., Livi, G. P., White, J. R., Adams, J. L., and Young, P. R. (1994) A protein kinase involved in the regulation of inflammatory cytokine biosynthesis. Nature, 372, 739-746.

(62) Leonard, W. J., and O'Shea, J. J. (1998) Jaks and STATs: biological implications. Annu Rev Immunol, 16, 293-322.

(63) O'Shea, J. J., Gadina, M., and Schreiber, R. D. (2002) Cytokine signaling in 2002: new surprises in the Jak/Stat pathway. Cell, 109 Suppl, S121-131.

(64) Lin, J. X., Migone, T. S., Tsang, M., Friedmann, M., Weatherbee, J. A., Zhou, L., Yamauchi, A., Bloom, E. T., Mietz, J., John, S., and et al. (1995) The role of shared receptor motifs and common Stat proteins in the generation of cytokine pleiotropy and redundancy by IL-2, IL-4, IL-7, IL-13, and IL-15. Immunity, 2, 331-339.

(65) Sheeran, P., and Hall, G. M. (1997) Cytokines in anaesthesia. Br J Anaesth, 78, 201-219. 
(66) Argiles, J. M., and Lopez-Soriano, F. J. (1999) The role of cytokines in cancer cachexia. Med Res $\operatorname{Rev}, 19,223-248$.

(67) Ladner, K. J., Caligiuri, M. A., and Guttridge, D. C. (2003) Tumor necrosis factor-regulated biphasic activation of NF-kappa B is required for cytokine-induced loss of skeletal muscle gene products. J Biol Chem, 278, 2294-2303.

(68) Langen, R. C., Schols, A. M., Kelders, M. C., Wouters, E. F., and Janssen-Heininger, Y. M. (2001) Inflammatory cytokines inhibit myogenic differentiation through activation of nuclear factorkappaB. Faseb J, 15, 1169-1180.

(69) Li, Y. P., and Reid, M. B. (2000) NF-kappaB mediates the protein loss induced by TNF-alpha in differentiated skeletal muscle myotubes. Am J Physiol Regul Integr Comp Physiol, 279, R11651170.

(70) Guttridge, D. C., Albanese, C., Reuther, J. Y., Pestell, R. G., and Baldwin, A. S., Jr. (1999) NFkappaB controls cell growth and differentiation through transcriptional regulation of cyclin D1. Mol Cell Biol, 19, 5785-5799.

(71) Fang, C. H., Li, B. G., James, J. H., King, J. K., Evenson, A. R., Warden, G. D., and Hasselgren, P. O. (2005) Protein breakdown in muscle from burned rats is blocked by insulin-like growth factor i and glycogen synthase kinase-3beta inhibitors. Endocrinology, 146, 3141-3149.

(72) Fang, C. H., Li, B., James, J. H., Yahya, A., Kadeer, N., Guo, X., Xiao, C., Supp, D. M., Kagan, R. J., Hasselgren, P. O., and Sheriff, S. (2007) GSK-3beta activity is increased in skeletal muscle after burn injury in rats. Am J Physiol Regul Integr Comp Physiol, 293, R1545-1551.

(73) Glass, D. J. (2003) Signalling pathways that mediate skeletal muscle hypertrophy and atrophy. Nat Cell Biol, 5, 87-90.

(74) Moore, S. F., van den Bosch, M. T., Hunter, R. W., Sakamoto, K., Poole, A. W., and Hers, I. (2013) Dual regulation of glycogen synthase kinase 3 (GSK3)alpha/beta by protein kinase C (PKC)alpha 
and Akt promotes thrombin-mediated integrin alphallbbeta3 activation and granule secretion in platelets. J Biol Chem, 288, 3918-3928.

(75) Doble, B. W., and Woodgett, J. R. (2003) GSK-3: tricks of the trade for a multi-tasking kinase. J Cell Sci, 116, 1175-1186.

(76) Cross, D. A., Alessi, D. R., Cohen, P., Andjelkovich, M., and Hemmings, B. A. (1995) Inhibition of glycogen synthase kinase-3 by insulin mediated by protein kinase B. Nature, 378, 785-789.

(77) Bodine, S. C., Latres, E., Baumhueter, S., Lai, V. K., Nunez, L., Clarke, B. A., Poueymirou, W. T., Panaro, F. J., Na, E., Dharmarajan, K., Pan, Z. Q., Valenzuela, D. M., DeChiara, T. M., Stitt, T. N., Yancopoulos, G. D., and Glass, D. J. (2001) Identification of ubiquitin ligases required for skeletal muscle atrophy. Science, 294, 1704-1708.

(78) Smith, I. J., and Dodd, S. L. (2007) Calpain activation causes a proteasome-dependent increase in protein degradation and inhibits the Akt signalling pathway in rat diaphragm muscle. Exp Physiol, 92, 561-573.

(79) Cabane, C., Englaro, W., Yeow, K., Ragno, M., and Derijard, B. (2003) Regulation of C2C12 myogenic terminal differentiation by MKK3/p38alpha pathway. Am J Physiol Cell Physiol, 284, C658-666.

(80) Cuenda, A., Rouse, J., Doza, Y. N., Meier, R., Cohen, P., Gallagher, T. F., Young, P. R., and Lee, J. C. (1995) SB 203580 is a specific inhibitor of a MAP kinase homologue which is stimulated by cellular stresses and interleukin-1. FEBS Lett, 364, 229-233.

(81) Penn, B. H., Bergstrom, D. A., Dilworth, F. J., Bengal, E., and Tapscott, S. J. (2004) A MyoDgenerated feed-forward circuit temporally patterns gene expression during skeletal muscle differentiation. Genes Dev, 18, 2348-2353. 
(82) Puri, P. L., Wu, Z., Zhang, P., Wood, L. D., Bhakta, K. S., Han, J., Feramisco, J. R., Karin, M., and Wang, J. Y. (2000) Induction of terminal differentiation by constitutive activation of p38 MAP kinase in human rhabdomyosarcoma cells. Genes Dev, 14, 574-584.

(83) Wu, Z., Woodring, P. J., Bhakta, K. S., Tamura, K., Wen, F., Feramisco, J. R., Karin, M., Wang, J. Y., and Puri, P. L. (2000) p38 and extracellular signal-regulated kinases regulate the myogenic program at multiple steps. Mol Cell Biol, 20, 3951-3964.

(84) Zetser, A., Gredinger, E., and Bengal, E. (1999) p38 mitogen-activated protein kinase pathway promotes skeletal muscle differentiation. Participation of the Mef2c transcription factor. J Biol Chem, 274, 5193-5200.

(85) Aronson, D., Wojtaszewski, J. F., Thorell, A., Nygren, J., Zangen, D., Richter, E. A., Ljungqvist, O., Fielding, R. A., and Goodyear, L. J. (1998) Extracellular-regulated protein kinase cascades are activated in response to injury in human skeletal muscle. Am J Physiol, 275, C555-561.

(86) Mitchel, R. E. (2004) The bystander effect: recent developments and implications for understanding the dose response. Nonlinearity Biol Toxicol Med, 2, 173-183.

(87) Azzam, E. I., and Little, J. B. (2004) The radiation-induced bystander effect: evidence and significance. Hum Exp Toxicol, 23, 61-65.

(88) Ramesh, R., Marrogi, A. J., Munshi, A., Abboud, C. N., and Freeman, S. M. (1996) In vivo analysis of the 'bystander effect': a cytokine cascade. Exp Hematol, 24, 829-838.

(89) Asur, R. S., Thomas, R. A., and Tucker, J. D. (2009) Chemical induction of the bystander effect in normal human lymphoblastoid cells. Mutat Res, 676, 11-16.

(90) Asur, R., Balasubramaniam, M., Marples, B., Thomas, R. A., and Tucker, J. D. (2010) Bystander effects induced by chemicals and ionizing radiation: evaluation of changes in gene expression of downstream MAPK targets. Mutagenesis, 25, 271-279. 
(91) Bissell, M. J., and Barcellos-Hoff, M. H. (1987) The influence of extracellular matrix on gene expression: is structure the message? J Cell Sci Suppl, 8, 327-343.

(92) Hei, T. K., Zhou, H., Ivanov, V. N., Hong, M., Lieberman, H. B., Brenner, D. J., Amundson, S. A., and Geard, C. R. (2008) Mechanism of radiation-induced bystander effects: a unifying model. J Pharm Pharmacol, 60, 943-950.

(93) Ghandhi, S. A., Ming, L., Ivanov, V. N., Hei, T. K., and Amundson, S. A. (2010) Regulation of early signaling and gene expression in the alpha-particle and bystander response of IMR-90 human fibroblasts. BMC Med Genomics, 3, 31.

(94) Jo, C., Jang, B. G., and Jo, S. A. (2009) MEK1 plays contrary stage-specific roles in skeletal myogenic differentiation. Cell Signal, 21, 1910-1917.

(95) Kaminska, B. (2005) MAPK signalling pathways as molecular targets for anti-inflammatory therapy--from molecular mechanisms to therapeutic benefits. Biochim Biophys Acta, 1754, 253262.

(96) Cuzzocrea, S., Genovese, T., Mazzon, E., Crisafulli, C., Di Paola, R., Muia, C., Collin, M., Esposito, E., Bramanti, P., and Thiemermann, C. (2006) Glycogen synthase kinase-3 beta inhibition reduces secondary damage in experimental spinal cord trauma. J Pharmacol Exp Ther, 318, 7989.

(97) Vrana, J., Currie, H., Han, A., and Boyd, J. (2014) Forecasting cell death dose-response from early signal transduction responses in vitro. Under Review Toxicol Sci. 